\title{
Rigidity versus flexibility for tight confoliations
}

\author{
THOMAS VOGEL
}

In their book [10] Y Eliashberg and W Thurston gave a definition of tight confoliations. We give an example of a tight confoliation $\xi$ on $T^{3}$ violating the Thurston-Bennequin inequalities. This answers a question from [10] negatively. Despite this, it is still possible to prove restrictions on homotopy classes of plane fields which contain tight confoliations.

The failure of the Thurston-Bennequin inequalities for tight confoliations is due to the presence of overtwisted stars. Overtwisted stars are particular configurations of Legendrian curves which bound a disc with finitely many punctures on the boundary. We prove that the Thurston-Bennequin inequalities hold for tight confoliations without overtwisted stars and that symplectically fillable confoliations do not admit overtwisted stars.

57R17, 57R30

\section{Introduction}

In [10] Eliashberg and Thurston explore the relationship between foliations and contact structures, on oriented 3-manifolds. Foliations, respectively contact structures on an oriented 3-manifold $M$ are locally defined by 1 -forms $\alpha$ such that $\alpha \wedge d \alpha \equiv 0$, respectively $\alpha \wedge d \alpha>0$ (more precisely this defines positive contact structures).

One of the main results of [10] is the following remarkable theorem.

Theorem 1.1 [10, Theorem 2.4.1] Suppose that a $C^{2}-$ foliation $\xi$ on a closed oriented 3-manifold is different from the product foliation of $S^{1} \times S^{2}$ by spheres. Then $\xi$ can be $C^{0}$-approximated by a positive contact structure.

In the main part of the proof of this theorem a given foliation on $M$ is modified so that the resulting plane field is somewhere integrable while it is a positive contact structure on other parts of $M$. This motivates the following definition.

Definition 1.2 A positive confoliation on a 3-manifold $M$ is a smooth plane field on $M$ which is locally defined by a $1-$ form $\alpha$ such that $\alpha \wedge d \alpha \geq 0$. We denote the region where $\xi$ is a contact structure by $H(\xi)$. 
Theorem 1.1 remains true when foliations are replaced by confoliations. Like in the case of foliations and contact structures the definition of confoliations can be generalized to higher dimensions (cf Altschuler and $\mathrm{Wu}$ [2] and [10]) but in this article we are only concerned with dimension 3. All plane fields appearing in this article will be oriented, in particular these plane fields have an Euler class $e(\xi) \in H^{2}(M ; \mathbb{Z})$.

In the last chapter of [10] Eliashberg and Thurston discuss several properties of foliations (tautness, absence of Reeb components) and contact structures (symplectic fillability, tightness) and what can be said about a contact structure approximating a taut or Reebless foliation. For example, they establish the following theorem.

Theorem (Eliashberg-Thurston [10]) If a contact structure $\xi$ on a closed 3-manifold is sufficiently close to a taut foliation in the $C^{0}$-topology, then $\xi$ is symplectically fillable and therefore tight.

Another result in this direction is due to V Colin.

Theorem (Colin [8]) A $C^{2}$-foliation without Reeb components on a closed oriented 3 -manifold can be $C^{0}$-approximated by tight contact structures.

In [13] J Etnyre shows that every contact structure (tight or not) may be obtained by a perturbation of a foliation with Reeb components. This result is implicitly contained in Mori [25]. Moreover, Etnyre improved Theorem 1.1 by showing that $C^{k}$-smooth foliations can be $C^{k}$-approximated by contact structures provided that $k \geq 2$, at least when the foliation is not a foliation without holonomy (his written account [11] will hopefully be available in the near future).

In order to understand better the relationship between geometric properties of foliations and properties of the contact structures approximating them, it is interesting to ask about properties of confoliations which appear in the approximation process. For example, the notion of symplectic fillability can be extended to confoliations in an obvious fashion.

The question how to generalize the notion of tightness is more complicated. One aim of this article is to clarify this point. The following definition is suggested in [10].

Definition 1.3 A confoliation $\xi$ on $M$ is tight if for every embedded disc $D \subset M$ such that

(i) $\partial D$ is tangent to $\xi$ and

(ii) $T D$ and $\xi$ are transverse along $\partial D$, 
and there is an embedded oriented disc $D^{\prime}$ satisfying the following requirements:

(1) $\partial D=\partial D^{\prime}$ with opposite orientations,

(2) $D^{\prime}$ is everywhere tangent to $\xi$ and

(3) $e(\xi)\left[D \cup D^{\prime}\right]=0$.

This definition is motivated by the following facts: If $\xi$ is a contact structure, then there are no surfaces tangent to $\xi$ and Definition 1.3 reduces to a definition of tightness for contact structures. In the case when $\xi$ is a foliation on a closed manifold Definition 1.3 is equivalent to the absence Reeb components by a theorem of Novikov [27]. Thus Definition 1.3 interpolates between tight contact structures and Reebless foliations. Moreover, the fact that symplectically fillable contact structures are tight generalizes to confoliations (we recall the definition of symplectic fillability in Section 2.4).

\section{Theorem 1.4 [10, Theorem 3.5.1] Symplectically fillable confoliations are tight.}

As pointed out in [10] there are inequalities imposing restrictions on the Euler class $e(\xi)$ of $\xi$ when $\xi$ is a tight contact structure or a Reebless foliation. Before we can state these inequalities we need one more definition.

Definition 1.5 Let $\gamma$ be a null-homologous knot in a confoliated manifold $(M, \xi)$ which is positively transverse to $\xi$. For each choice $F$ of an oriented Seifert surface of $\gamma$ we define the self linking number $\operatorname{sl}(\gamma, F)$ of $\gamma$ as follows. Choose a nowhere vanishing section $X$ of $\left.\xi\right|_{F}$ and let $\gamma^{\prime}$ be the knot obtained by pushing $\gamma$ off itself by $X$. Then

$$
\operatorname{sl}(\gamma, F)=\gamma^{\prime} \cdot F
$$

One may easily show that $\operatorname{sl}(\gamma, F)$ depends only on $[F] \in H_{2}(M, \gamma ; \mathbb{Z})$.

In [4] $\mathrm{D}$ Bennequin proved an inequality between $\operatorname{sl}(\gamma)$ of a transverse knot in the standard contact structure $\operatorname{ker}(d z+x d y)$ on $\mathbb{R}^{3}$ and the Euler number of a Seifert surface of $\gamma$. This inequality was extended to all tight contact structures by Eliashberg in [9]. From Thurston's work in [31] it follows that the same inequalities hold for surfaces in foliated manifolds without Reeb components. We summarize these results as follows.

Theorem 1.6 (Eliashberg [9], Thurston [31]) Let $\xi$ be a tight contact structure or a foliation without Reeb components on a closed manifold $M$ (different from a foliation by spheres) and $F \subset M$ an embedded oriented surface.

(a) If $F \simeq S^{2}$, then $e(\xi)[F]=0$.

(b) If $\partial F=\varnothing$ and $F \not 4 S^{2}$, then $|e(\xi)[F]| \leq-\chi(F)$.

(c) If $\partial F \neq \varnothing$ is positively transverse to $\xi$, then $\operatorname{sl}(\gamma,[F]) \leq-\chi(F)$. 
The inequalities stated in this theorem are referred to as Thurston-Bennequin inequalities. In particular, they imply that only finitely many classes in $H^{2}(M ; \mathbb{Z})$ are Euler classes of tight contact structures or foliations without Reeb components. Foliations by spheres violate (a) and we exclude such foliations from our discussion.

It was conjectured [10, Conjecture 3.4.5] that tight confoliations satisfy the ThurstonBennequin inequalities. In this article we show that (a) is true for tight confoliations and (c) holds when $F$ is a disc. On the other hand we give an example of a tight confoliation $\xi^{\prime}$ on $T^{3}$ which violates (b) and (c) for surfaces which are not simply connected. Therefore every contact structure which is close to $\xi^{\prime}$ must be overtwisted. This yields a negative answer to Question 1 on page 63 of [10]. The construction of $\left(T^{3}, \xi^{\prime}\right)$ is based on the classification of tight contact structures on $T^{2} \times[0,1]$ due to E Giroux and the following:

Theorem 5.1 Let $\xi$ be a tight confoliation on $M$ and $T_{0}, T_{1} \subset \partial M$ two incompressible tori such that $\xi$ is a contact structure near $T_{i}$ and $T_{i}(\xi), i=0,1$, is linear. Let $\psi: T_{0} \rightarrow T_{1}$ be an orientation preserving diffeomorphism, where $T_{0}$, respectively $T_{1}$, is cooriented by the outward, respectively inward, pointing normal vector field.

The manifold $M^{\prime}=M \cup T_{0} \times[0,1] / \sim$, where $\sim$ identifies $T_{0} \simeq T_{0} \times\{0\}$, respectively $T_{1} \simeq T_{0} \times\{1\}$, using the identity, respectively $\psi$, carries a tight confoliation $\xi^{\prime}$ such that $\left.\xi^{\prime}\right|_{M^{\prime} \backslash T_{0} \times[0,1]}$ is isomorphic to $\left.\xi\right|_{M \backslash T_{0} \cup T_{1}}$.

This indicates that tight confoliations are much more flexible objects than tight contact structures or foliations without Reeb components. For example, infinitely many elements of $H^{2}\left(T^{3} ; \mathbb{Z}\right)$ are Euler classes of tight confoliations. Nevertheless, tight confoliations have some rigidity properties. In addition to the Thurston-Bennequin inequalities for simply connected surfaces we show the following theorem.

Theorem 4.1 Let $M$ be a manifold carrying a tight confoliation $\xi$ and $B \subset M$ a closed embedded ball in $M$. There is a neighbourhood of $\xi$ in the space of plane fields with the $C^{0}$-topology such that $\left.\xi^{\prime}\right|_{B}$ is tight for every contact structure $\xi^{\prime}$ in this neighbourhood of $\xi$.

This theorem leads to restrictions on the homotopy class of plane fields which contain tight confoliations. For example, only one homotopy class of plane fields on $S^{3}$ contains a tight confoliation by Eliashberg's classification of tight contact structures on balls together with Theorem 4.1. For the proof of Theorem 4.1 we study the characteristic foliation $S(\xi)=T S \cap \xi$ on embedded spheres $S \subset M$ (we generalize the notion of taming functions introduced in [9] to confoliations and use results from Giroux [17]). 
Motivated by the example $\left(T^{3}, \xi^{\prime}\right)$ we define the notion of an overtwisted star. Roughly speaking, an overtwisted star on an embedded surface $F$ is a domain in $F$ whose interior is homeomorphic to a disc, the boundary of this domain consists of Legendrian curves and all singularities on the boundary have the same sign. The main difference between overtwisted stars and overtwisted discs (ie discs $D$ with the properties required in Definition 1.3 which are not neutralized by an integral disc $D^{\prime}$ ) is that the set theoretic boundary of an overtwisted star may contain closed leaves or quasi-minimal sets of the characteristic foliation.

An example of an overtwisted star is shown in Figure 16 in Section 5. It will be clear from the definition of overtwisted stars that contact structures which admit overtwisted stars are not tight, ie they are overtwisted in the usual sense. The following results indicate that tightness in the sense of Definition 1.3 together with the absence of overtwisted stars is the right generalization of tightness to confoliations and (since the notion of strong tightness is already used [10]), we will call a tight confoliation without overtwisted stars s-tight.

Theorem 6.2 Let $\xi$ be an oriented s-tight confoliation which is not a foliation by spheres. Every embedded surface $F$ whose boundary is either empty or positively transverse to $\xi$ satisfies the following relations.

(a) If $F \simeq S^{2}$, then $e(\xi)[F]=0$.

(b) If $\partial F=\varnothing$ and $F \not S^{2}$, then $|e(\xi)[F]| \leq-\chi(F)$.

(c) If $\partial F \neq \varnothing$ is positively transverse to $\xi$, then $\operatorname{sl}(\gamma,[F]) \leq-\chi(F)$.

Moreover, Theorem 1.4 can be refined as follows.

\section{Theorem 6.9 Symplectically fillable confoliations are s-tight.}

The proof of this theorem implies that a confoliation which admits overtwisted stars can be approximated by an overtwisted contact structure.

This article is organized as follows: In Section 2 we recall facts about confoliations and characteristic foliations. Section 3 contains a discussion of several methods for the manipulation of characteristic foliation on embedded surfaces: We generalize the elimination lemma to confoliations and we discuss several surgeries of surfaces when integral discs of $\xi$ intersect the surface in a cycle. In Section 4 we prove Theorem 4.1 and other rigidity results for tight confoliations. In Section 5 we develop a construction of tight confoliations which yields a tight confoliation on $T^{3}$ violating the ThurstonBennequin inequalities. 
In Section 6 we discuss overtwisted stars and establish the Thurston-Bennequin inequalities for tight confoliations without overtwisted stars. Moreover, we prove that symplectically fillable confoliations do not admit overtwisted stars.

Throughout this article $M$ will be a closed connected oriented 3-manifold and $\xi$ will always denote a smooth oriented plane field on $M$.

Acknowledgements The author started working on this project in the fall of 2006 during a stay at Stanford University, the financial support provided by the "Deutsche Forschungsgemeinschaft" is gratefully acknowledged. It is a pleasure for me to thank Y Eliashberg for his support, hospitality and interest. Moreover, I would like to thank V Colin and J Etnyre for helpful conversations. Finally, I thank the referee for the careful reading of the manuscript.

\section{Characteristic foliations, nonintegrability and tightness}

In this section we recall some definitions, notation and facts which will be used throughout this paper. Most notions discussed here are generalizations of definitions which are well-known in the context of contact structures and foliations (see for example Aebischer et al [1], Candel and Conlon [5; 6], Etnyre [12], Geiges [15], Giroux [16] and the references therein).

\subsection{Characteristic foliations on surfaces}

We consider an embedded oriented surface $F$ in a confoliated 3 -manifold $(M, \xi)$ and we assume that $\xi$ is cooriented. The singular foliation $F(\xi):=\xi \cap T F$ is called the characteristic foliation of $F$. The leaves of the characteristic foliation are examples of Legendrian curves, ie curves tangent to $\xi$.

The following convention is used to orient $F(\xi)$ : Consider $p \in F$ such that $F_{p}(\xi)$ is one-dimensional. For $X \in F_{p}(\xi)$ we choose $Y \in \xi(p)$ and $Z \in T_{p} F$ such that $X, Y$ represents the orientation of $\xi(p)$ and $X, Z$ induces the orientation of the surface. Then $X$ represents the orientation of the characteristic foliation if and only if $X, Y, Z$ is a positive basis of $T_{p} M$.

By this convention the characteristic foliation points out $F$ along boundary components of $F$ which are positively transverse to $\xi$. An isolated singularity of $F(\xi)$ is called elliptic respectively hyperbolic when its index is +1 , respectively -1 . A singularity is positive if the orientation of $\xi$ coincides with the orientation of $F$ at the singular point and negative otherwise. We denote the number of positive/negative elliptic singularities by $e_{ \pm}(F)$ and the number of positive/negative hyperbolic singularities is $h_{ \pm}(F)$. 
Because we shall frequently assume that a surface in $M$ is in general position with respect to a given confoliation let us recall the jet-transversality theorem (cf for example Chapter 3 of [21]).

Theorem Let $F, M$ be compact smooth manifolds and $\Sigma$ a smooth submanifold of the space of $k$-jets $J^{k}(F, M)$ of maps from $F$ to $M$ for $k=1,2, \ldots, \infty$. The set of $C^{l}$-maps $f: F \rightarrow M$ with $l>k$ such that the $k$-jet extension $j^{k}(f): F \rightarrow J^{k}(F, M)$ of $f$ (which maps $q \in F$ to the $k$-jet of $f$ at $q$ ) is transverse to $\Sigma$ is residual in the space of all $C^{l}$-maps with respect to the strong $C^{l}$-topology.

Recall that $J^{k+1}(F, M)$ is the total space of a fibration over $J^{k}(F, M)$ and that $J^{0}(F, M)=F \times M$. Given a smooth plane field $\xi$ on a 3 -manifold $M$ we apply this theorem to the submanifold $\Sigma_{\xi} \subset J^{1}(F, M)$ whose intersection with the fiber over $(q, p) \in F \times M$ is

$$
\left\{1 \text {-jets of maps } f: F \rightarrow M \text { with } f(q)=p \text { and } D f\left(T_{q} F\right)=\xi(p)\right\} .
$$

The codimension of $\Sigma_{\xi} \subset J^{1}(F, M)$ is two and the smoothness of $\xi$ implies that $\Sigma_{\xi}$ is smooth. Hence the characteristic foliation on an embedded surface has isolated singularities if the 1-jet extension of the embedding is transverse to $\Sigma_{\xi}$ and generic embeddings have this property when they are sufficiently smooth.

The jet-transversality theorem does also apply to 1 -parameter families $F_{t}, t \in[0,1]$ of embedded surfaces. Such a family may be perturbed such that the singularities of the characteristic foliation on the resulting surfaces $F_{t}^{\prime}$ are all nondegenerate except for finitely many times $t_{1}, \ldots, t_{k} \in[0,1]$ and exactly one singularity on $F_{t_{i}}^{\prime}$ is of birth-death type (and hence isolated) while all other singularities on $F_{t_{i}}^{\prime}$ are nondegenerate.

After an additional perturbation of an embedded surface we can assume that there are no leaves connecting hyperbolic singularities. Similarly, after perturbing a 1-parameter family we can achieve that leaves connecting hyperbolic singularities occur only finitely many times and hyperbolic singularities are never connected with singularities of birthdeath type. We will frequently use these genericity properties.

\subsection{Properties of foliations on surfaces}

In this section we review basic definitions and properties of foliations on surfaces. Details can be found in Nikolaev and Zhuzhoma [26] and Aranson, Belitsky and Zhuzoma [3]. We will always assume that $F$ is a closed oriented surface and $\mathcal{F}$ is an oriented smooth foliation with isolated singularities, these assumptions are satisfied for the characteristic foliation on generically embedded oriented surfaces in confoliated manifolds (and also for surfaces from generic 1-parameter families). 
A subset $U$ of $F$ is saturated if it is a union of leaves and singularities of $\mathcal{F} . U$ is minimal if it is closed and saturated and every subset $V$ of $U$ with the same properties is either empty or $U=V$. Closed leaves and singularities of $\mathcal{F}$ are examples of minimal sets.

A closed saturated set $U$ is dynamically irreducible if it cannot be represented as a union of two disjoint nonempty closed sets which are saturated. One distinguishes two classes of dynamically irreducible sets: $U$ is exceptional if for every point $p \in U$ which is not a singularity of $\mathcal{F}$, there is a neighbourhood $V$ such that the only leaf in $U$ which intersects $V$ is the leaf through $p$ and this intersection consists of one segment of that leaf. Examples of this type are cycles consisting of hyperbolic singularities and stable/unstable leaves. If a dynamically irreducible set is not exceptional it is said to be quasi-minimal.

The different types of saturated subsets arise as limit sets of leaves of $\mathcal{F}$ : Fix a vector field orienting $\mathcal{F}$ and let $\psi_{t}$ be the corresponding flow. The $\omega$-limit $\Omega(x)$ set of $x \in F$ is defined by

$$
\Omega(x):=\bigcap_{\{\tau>0\}} \overline{\psi_{[\tau, \infty)}(x)}
$$

The $\alpha$-limit set $\mathcal{A}(x)$ of $x$ is obtained when the orientation of $\mathcal{F}$ is reversed. Note that $\Omega(x)$ and $\mathcal{A}(x)$ depend only on the leaf $\gamma$ through $x$, so we can also write $\Omega(\gamma)$ and $\mathcal{A}(\gamma)$. A leaf $\gamma$ of $F$ is recurrent if $\gamma$ is contained in $\mathcal{A}(\gamma)$ and $\Omega(\gamma)$.

We assumed that $\mathcal{F}$ is smooth with isolated singularities and $F$ is compact. By Theorem 2.6.1 from [26] the limit set $\Omega(x)$ belongs to one of the following classes:

- fixed points,

- closed leaves,

- cycles consisting of singular points and leaves connecting them and

- quasi-minimal sets.

Closures of nonperiodic recurrent trajectories are quasi-minimal sets. According to Theorem 2.3.3 of [26] a quasi-minimal set $U$ contains an uncountable number of recurrent leaves all of which are dense in $U$.

The intersection between two different quasi-minimal sets cannot contain a recurrent orbit by Maier's theorem [26, Theorem 2.4.1] and the number of quasi-minimal sets of $F(\xi)$ is bounded by the genus of $F$ according to Theorem 2.4.5 in [26]. 


\section{3 (Non-)Integrability}

The condition that $\xi$ is a confoliation can be interpreted in geometric terms. The following interpretation can be found in [10].

Let $D$ be a closed disc of dimension 2 and $\xi$ a positive confoliation transverse to the fibers of $\pi: D \times \mathbb{R} \rightarrow D$. Then $\xi$ can be viewed as a connection. We assume in the following that this connection is complete, ie for every differentiable curve $\sigma$ in $D$ there is a horizontal lift of $\sigma$ starting at a given point in the fiber over the starting point of $\sigma$.

We consider the holonomy of the characteristic foliation on $\pi^{-1}(\partial D)$

$$
h_{\partial D}: \pi^{-1}(p) \simeq \mathbb{R} \rightarrow \mathbb{R} \simeq \pi^{-1}(p)
$$

where $h_{\partial D}(x)$ is defined as the parallel transport of $x \in \mathbb{R}$ along $\partial D$.

Lemma 2.1 [10, Lemma 1.3.4] If the confoliation $\xi$ on $\pi: D \times \mathbb{R} \rightarrow D$ defines a complete connection, then $h_{\partial D}(x) \leq x$ for all $x \in \pi^{-1}(p)$ and $p \in \partial D$. Equality holds for all $x \in \pi^{-1}(p)$ if and only if $\xi$ is integrable.

If $D=D \times\{0\}$ is tangent to $\xi$, then the germ of the holonomy is well defined without any completeness assumption and $h_{\partial D}(x) \leq x$ for all $x$ in the domain of $h_{\partial D}$. The germ of $h_{\partial D}$ coincides with the germ of the identity if and only if a neighbourhood of $D$ is foliated by discs.

Of course, the second part of the lemma applies to the case when one considers only the part lying above or below $D \times\{0\} \subset D \times \mathbb{R}$. A consequence of Lemma 2.1 is the following generalization of the Reeb stability theorem to confoliations.

Theorem 2.2 [10, Proposition 1.3.9] Let $M$ be a closed oriented manifold carrying a positive confoliation $\xi$. Suppose that $S$ is an embedded sphere tangent to $\xi$. Then $(M, \xi)$ is diffeomorphic to the product foliation on $S^{2} \times S^{1}$ by spheres.

Foliations by spheres appear as exceptional case in some theorems. They will therefore be excluded from the discussion.

Another useful geometric interpretation of the confoliation condition can be found on page 4 in [10] (and many other sources): Let $X$ be a Legendrian vector field and $F$ a surface transverse to $X$. We consider the family $F_{t}(\xi)$ of foliations where $F_{t}$ is the image of $F$ under the time- $t$-flow of $X$. Using this flow we identify $F_{t}$ with $F_{0}$. Then at each point the line $F_{t}(\xi)$ rotates in a monotone sense when $t$ is increasing. If $\xi$ is a contact structure, then the rotation is strictly monotone. This interpretation 
is useful when one wants extends confoliations along leaves of a foliation of rank 1 which is Legendrian where the confoliation is already defined.

We define the fully foliated part of a confoliation $\xi$ on $M$ as the complement of

$$
\{x \in M \mid \text { there is a Legendrian curve connecting } x \text { to } H(\xi)\} \text {. }
$$

If $\gamma$ is a Legendrian curve in a leaf of $\xi$ and $A \simeq \gamma \times(-\delta, \delta), \delta>0$ an annulus transverse to the leaf such that $\gamma=\gamma \times\{0\}$, then we will consider several types of holonomy $h_{\gamma}$ of the characteristic foliation on $A\left(h_{\gamma}\right.$ is the Poincaré-return map defined on a neighbourhood of $\gamma(0)$ in $\gamma(0) \times(-\delta, \delta)$ ).

- We say that $\gamma$ has linear holonomy or nontrivial infinitesimal holonomy along $\gamma$ if $h_{\gamma}^{\prime}(0) \neq 0$.

- The holonomy along $\gamma$ is sometimes attractive if there are sequences $\left(x_{n}\right)$ and $\left(y_{n}\right)$ which converge to zero such that $x_{n}>0>y_{n}$ and

$$
h_{\gamma}\left(x_{n}\right)<x_{n}, \quad h_{\gamma}\left(y_{n}\right)>y_{n} \quad \text { for all } n \in \mathbb{N} .
$$

\subsection{Tightness of confoliations}

In this section we summarize several facts about tight confoliations. We shall always assume that $\xi$ is a tight confoliation but it is not a foliation by spheres.

If $(M, \xi)$ is tight and $D \subset M$ is an embedded disc such that $\partial D$ is tangent to $\xi$ and $\left.\xi\right|_{\partial D}$ is transverse to $T D$, then the disc $D^{\prime}$ whose existence is guaranteed by Definition 1.3 is uniquely determined. Otherwise there would be a sphere tangent to $\xi$ and $\xi$ would be a foliation by spheres by Theorem 2.2. But we explicitly excluded this case.

The definition of tightness refers to smoothly embedded discs but of course it has implications for discs whose boundary contains corners and slightly more generally for unions of such discs.

Lemma 2.3 Suppose that $(M, \xi)$ is a tight confoliation and $S \subset M$ is an embedded sphere such that the characteristic foliation $S(\xi)=T S \cap \xi$ has only nondegenerate hyperbolic singularities along a connected cycle $\gamma$ of $S(\xi)$. Then there are immersed discs $D_{i}^{\prime}, i=1, \ldots k$ in $M$ which are tangent to $\xi$ and

$$
\partial\left(\bigcup_{i=1}^{k} D_{i}^{\prime}\right)=\gamma
$$


This follows by considering $C^{\infty}$-small perturbations of $S$ such that $\gamma$ is approximated by closed leaves of the characteristic foliation of the perturbed sphere. More precisely, let $p$ be a corner of $\gamma$. We want to perturb $S$ in a neighbourhood of $p$ such that we obtain a cycle which is close to a part of $\gamma$ and containing less corners. For this we view $\xi$ as a connection on the normal bundle of a neighbourhood $U$ of a hyperbolic singularity $p \in \gamma$. Let $\sigma, \tau \subset \gamma \cap U_{p}$ be segments of the stable or unstable leaf of $p$. We will replace $\sigma \cup \tau$ by a smooth Legendrian curve which coincides with $\sigma \cup \tau$ outside a small neighbourhood of $p$.

We will continue to say that a disc bounds the cycle $\gamma$ although the "disc" might have corners or be a pinched annulus, for example.

The most important criterion to prove tightness is symplectic fillability; see Theorem 1.4. Let us recall the definition of symplectic fillability.

Definition 2.4 A positive confoliation $\xi$ on a closed oriented manifold $M$ is symplectically fillable if there is a compact symplectic manifold $(X, \omega)$ such that

(i) $\left.\omega\right|_{\xi}$ is nondegenerate and

(ii) $\partial X=M$ as oriented manifolds where $X$ is oriented by $\omega \wedge \omega$.

In this definition we use the "outward normal first" convention for the orientation of the boundary. There are several different notions of symplectic fillings and Definition 2.4 is often referred to as weak symplectic filling. It is clear from Theorem 1.4 (and Theorem 6.9) that the existence of a symplectic filling is an important property of a confoliation.

Theorem 1.4 can be applied to some noncompact manifolds.

Proposition 2.5 [10, Proposition 3.5.6] If a confoliation $\xi$ is transverse to the fibers of the projection $\mathbb{R}^{3} \rightarrow \mathbb{R}^{2}$ and if the induced connection is complete, then $\xi$ is tight.

In [10] one can find an example which shows that the completeness condition can not be dropped. Note that if $(M, \xi)$ is symplectically fillable, then the same is true for confoliations $\xi^{\prime}$ which are sufficiently close to $\xi$ in the $C^{0}$-topology.

\section{Properties and modifications of characteristic foliations}

The characteristic foliations on embedded surfaces in manifolds with contact structures have several properties reflecting the positivity of the contact structure. Moreover, 
there are methods to manipulate the characteristic foliation by isotopies of the surface. Similar remarks apply when $\xi$ is a foliation. In this section we generalize this to the case when $\xi$ is a confoliation. If $\xi$ is tight, then there are more restrictions on characteristic foliation. Some of these additional restrictions shall be discussed in Section 4.

\subsection{Neighbourhoods of elliptic singularities}

By our orientation convention positive elliptic singular points lying in the contact region are sources. The following lemma shows how to interpret this statement for confoliations.

Lemma 3.1 Let $(M, \xi)$ be a confoliated manifold and $F$ an immersed surface whose characteristic foliation has a nondegenerate positive elliptic singularity $p$.

There is an open disc $p \in D \subset F$ such that each leaf of the characteristic foliation on $D$ is either a circle or there is a closed transversal of $F(\xi)$ through the leaf. If $p$ is positive respectively negative and $\partial D$ is transverse to $F(\xi)$, then $F(\xi)$ points outwards respectively inwards.

Proof We fix a defining form $\alpha$ for $\xi$ on a neighbourhood of $p$ and we denote the restriction of $\alpha$ to $F$ by $\alpha_{F}$. If $d \alpha_{F}(p) \neq 0$, then $p$ lies in the interior of the contact region and the claim follows from [16]. Now assume $d \alpha_{F}(p)=0$ and fix coordinates $x, y$ on $F$ near $p$ such that $x(p)=y(p)=0$. The Taylor expansion for $\alpha_{F}$ around $p$ is given by $\alpha_{F}=(a x+b y) d x+(b x+c y) d y+o\left(x^{2}+y^{2}\right)$. Hence $F(\xi)$ is transverse to the gradient vector field $R$ of the Morse function $F(x, y)=a x^{2} / 2+b x y+c y^{2} / 2$ on a small neighbourhood of $p$ in $F$ (the index of $p$ as critical point of $F$ is 0 ).

In the following we assume that $p$ is positive and $R$ points away from $p$ and coorients $\xi$ away from $p$ (the other cases are similar). The Poincaré return map of the characteristic foliation is well defined on a small neighbourhood of $p$ in a fixed radial line starting at the origin (see Marsden [24] for example) and $F(\xi)$ is oriented clockwise near $p$ by our orientation convention. We want to show that the Poincare return map is nondecreasing when the orientation of the radial line points away from $p$.

Fix a vector field $Z$ coorienting both $F$ and $\xi$. We write $D_{z}$ for the image of $F$ under the time $z$-flow of $Z$. We may assume that the tangencies of $D_{z}$ and $\xi$ are exactly the points on the flow line $\gamma_{p}$ of $Z$ through $p$.

We extend $R$ to a vector field on a neighbourhood of $p$ tangent to $D_{z}$ such that it remains transverse to $\xi$ on $U \backslash \gamma_{p}$. Then the vector field $T=z Z+R$ is transverse 
to $\xi$ on $\{z \geq 0\} \backslash\{p\} \subset U$. The flow of $T$ exists for all negative times $t$ and every flow line of $T$ approaches $p$ as $t \rightarrow-\infty$. Since $d \alpha_{F}(p)=0$ and $p$ is elliptic there are local coordinates $x, y$ on $D$ around $p$ such that $p$ corresponds to the origin and

$$
\alpha=d z+(x d x+\lambda y d y)+\tilde{\alpha}
$$

where $\lambda>0$ and $\tilde{\alpha}$ denotes a $1-$ form such that $\tilde{\alpha} \cdot\left(x^{2}+y^{2}+|z|\right)^{-1}$ remains bounded when one approaches the origin.

We choose a closed embedded disc $D^{\prime}$ in $\{z \geq 0\}$ which is transverse to $T$ and $D$ such that $\partial D^{\prime}=\partial D$ and $D \cup D^{\prime}$ bound a closed half ball $B$. This half ball is identified with a Euclidean half ball of radius 1 and we fix spherical coordinates $\rho, \vartheta, \phi$ (where $\rho$ denotes the distance of a point from the origin, $\vartheta$ is the angle between $\gamma_{p}$ and the straight line connecting the point with the origin) such that $T$ corresponds to $\rho \partial_{\rho}$. In this coordinate system

$$
\begin{aligned}
\alpha= & \left(\cos (\vartheta)+\rho \sin ^{2}(\vartheta)\left(\cos ^{2}(\varphi)+\lambda \sin ^{2}(\varphi)\right) d \rho\right. \\
& +\left(\rho \sin (\vartheta)+\rho^{2} \cos (\vartheta) \sin (\vartheta)\left(\cos ^{2}(\varphi)+\lambda \sin ^{2}(\varphi)\right) d \vartheta\right. \\
& +\left(\rho^{2} \sin ^{2}(\vartheta)(\lambda-1) \cos (\varphi) \sin (\varphi)\right) d \varphi+\widetilde{\alpha}
\end{aligned}
$$

and $\tilde{\alpha} \cdot\left(\rho^{2} \sin ^{2}(\vartheta)+|\rho \cos (\vartheta)|\right)^{-1}$ remains bounded when one approaches the origin. Thus $\alpha$ extends to a 1 -form of class $C^{\infty}$ on $[0,1] \times[0,2 \pi] \times[0, \pi / 2]$ (the first factor corresponds to $\rho$, the second factor to $\varphi$ and the third factor to $\vartheta$ ).

Consider a closed disc $D^{\prime \prime}$ lying in the interior of $D^{\prime}$. We identify the union of all flow lines of $T$ which intersect $D^{\prime \prime}$ with $D^{\prime \prime} \times(0,1]$ such that the second factor corresponds to flow lines of $T$. On $D^{\prime \prime} \times(0,1]$ the factor $\cos (\vartheta)$ is bounded away from 0 . By (3) the plane field $\operatorname{ker}(\alpha)$ extends to a smooth plane field on $D^{\prime \prime} \times[0,1]$ such that $D^{\prime \prime} \times\{0\}$ is tangent to the extended plane field.

The holonomy of the characteristic foliation on $\partial D^{\prime \prime} \times[0,1]$ is nonincreasing by Lemma 2.1 when $\partial D^{\prime \prime} \times\{0\}$ is oriented as the boundary of $D^{\prime \prime}$. Our orientation assumptions at the beginning of the proof imply that the characteristic foliation on $\partial D^{\prime} \times(0,1]$ is oriented in the opposite sense. This implies that the Poincaré-return map of the characteristic foliation around $p$ is nondecreasing.

\subsection{Legendrian polygons}

In the proof of rigidity theorems for tight confoliations and also in Section 6 we will use the notion of basins and Legendrian polygons. In this section we adapt the definitions from Eliashberg [9]. 
Definition 3.2 A Legendrian polygon $(Q, V, \alpha)$ on a compact embedded surface $F$ in a confoliated manifold $(M, \xi)$ is a triple consisting of a connected oriented surface $Q$ with piecewise smooth boundary, a finite set $V \subset \partial Q$ and a differentiable map $\alpha: Q \backslash V \rightarrow F$ which is an orientation preserving embedding on the interior such that

(i) corners of $Q$ are mapped to singular points of $F(\xi)$,

(ii) smooth pieces of $\partial Q$ are mapped onto smooth Legendrian curves on $F$ and

(iii) for points $v \in V$ the image $\alpha\left(b_{ \pm}\right)$of the two segments $b_{ \pm} \subset \partial Q \backslash V$ which end at $v$ have the same $\omega$-limit set $\gamma_{v}$ and $\gamma_{v}$ is not a singular point of $F(\xi)$.

A point $x \in \partial Q \backslash V$ is a vertex if $\alpha(x)$ is a singular point of $F(\xi)$. It is

- a pseudovertex if $\alpha(x)$ is a hyperbolic singularity and $\left.\alpha\right|_{\partial Q}$ is smooth at $\alpha(x)$,

- a corner if $\alpha(x)$ is a hyperbolic singularity and $\alpha$ is not smooth near $x$ and

- an elliptic vertex if $\alpha(x)$ is elliptic.

The elements of $V$ are called virtual vertices,

The points in $V$ should be thought of as missing vertices in the boundary of $Q$. Figure 1 shows the image $\alpha(Q)$ of a Legendrian polygon $(Q, V, \alpha)$ where $Q$ is a disc, $V=\{v\} \subset \partial Q$ and the corresponding ends of $\partial Q \backslash\{v\}$ are mapped to leaves of the characteristic foliation whose $\omega$-limit set is the closed leaf $\gamma_{v}$. There are three pseudovertices.

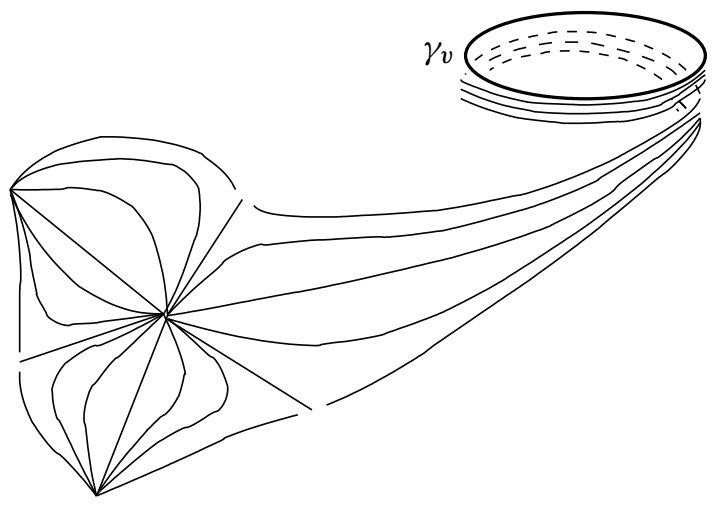

Figure 1

The following definition generalizes the notion of injectivity of a Legendrian polygon to the context of confoliations. 
Definition 3.3 A Legendrian polygon $(Q, V, \alpha)$ identifies pseudovertices if

- there are two pseudovertices $w_{1}, w_{2}$ with $\alpha\left(w_{1}\right)=\alpha\left(w_{2}\right)$ or

- there are pseudovertices $w_{1}, \ldots, w_{k}$ and corners $w_{1}^{\prime}, \ldots, w_{k}^{\prime}$ such that for each $i=1, \ldots, k$ there is a segment of $\partial Q$ whose interior contains only corners and which connects $w_{i}$ to $w_{i}^{\prime}, \alpha\left(w_{i}\right)=\alpha\left(w_{i+1}^{\prime}\right)$ for $i=1, \ldots, k-1$ and $\alpha\left(w_{k}\right)=\alpha\left(w_{1}^{\prime}\right)$.

The union of stable and unstable separatrices connecting the hyperbolic singularities $w_{i}$ to $w_{i+1}, i=1, \ldots, l$ and $w_{l}$ to $w_{1}$ form a cycle $\gamma_{w_{1} \ldots w_{l}}$. (This cycle may or may not be trivial of $l=2$.)

A Legendrian polygon which does not identify pseudovertices is called injective.

Notice that $\alpha$ may identify vertices even if $(Q, V, \alpha)$ is injective. An example of a nontrivial cycle $\gamma_{w_{1}} w_{2} w_{3}$ which can arise from a noninjective Legendrian polygon is shown Figure 2. The bold curves correspond to the image of $\alpha(\partial Q)$, the thin curves represent the characteristic foliation on $\alpha(Q)$.

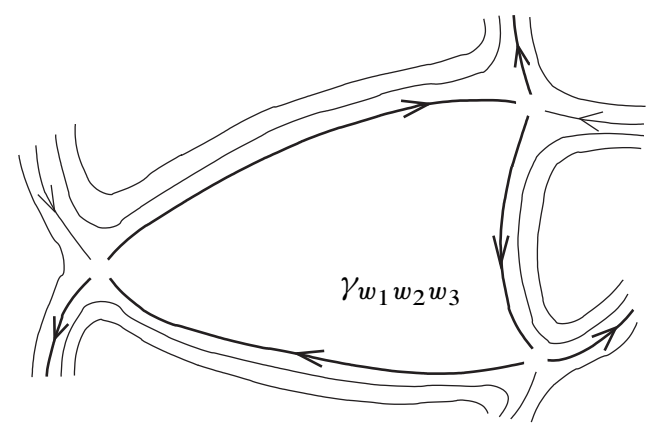

Figure 2

In the following lemma we require that the characteristic foliation $F(\xi)$ surface $F \subset M$ has the following properties:

- The singular points are isolated and either nondegenerate or of birth-death type. There is at most one birth-death type singularity.

- A singularity of birth-death type is contained in the interior of $H(\xi)$ or in the interior of $M \backslash H(\xi)$. Moreover, the closure of stable or unstable leaf of hyperbolic singularities do not contain singularities of birth-death type.

For example, these assumptions are satisfied by surfaces from generic 1-parameter families of embedded surfaces. 
Lemma 3.4 Let $F \subset M$ be a surface and $\xi$ a confoliation on $M$ such that $\partial F$ is transverse to $\xi$ and the characteristic foliation points inwards along $\partial F$. Assume that $U \subset F$ is a submanifold of dimension 2 such that every component of $\partial U$ is either is tangent to $F(\xi)$ or transverse to $\xi$ and the characteristic foliation points outwards.

Let $B(U)$ be the union of all leaves of $F(\xi)$ which intersect $U$. Then $\overline{B(U)}$ is the image $\alpha(Q \backslash V)$ of a Legendrian polygon $(Q, V, \alpha)$.

Proof A preliminary candidate for $(Q, V, \alpha)$ is $Q_{0}:=U, V_{0}=\varnothing$ and $\alpha_{0}$ the inclusion of $Q_{0}$. We will define vertices and edges of $Q$ and we will glue 1 -handles to components of $\partial Q_{0}$. The existence of $\alpha$ will be immediate once the correct polygon with all pseudovertices, corners and elliptic singularities and $V$ are defined.

Each intersection of $\partial U$ with a stable leaf of a hyperbolic singularity of $F(\xi)$ defines a vertex of $Q_{0}$. These vertices form a subset $P_{0} \subset \partial Q_{0}$ which will serve as a first approximation for the set of pseudovertices. For $p \in P_{0}$ we denote the corresponding hyperbolic singularity of $F(\xi)$ by $\alpha(p)$.

First, we consider the boundary components $\Gamma$ of $Q_{0}$ which are transverse to $F(\xi)$ and $\Gamma \cap P_{0}=\varnothing$. All leaves of $F(\xi)$ passing through $\Gamma$ have the same $\omega$-limit set $\Omega(\Gamma)$ (cf Proposition 14.1.4 in Katok and Hasselblatt [23]).

We claim that $\Omega(\Gamma)$ is an elliptic singularity or a cycle: Assume that $\Omega(\Gamma)$ is quasiminimal. According to Theorem 2.3.3 in [26] there is a recurrent leaf $\gamma$ which is dense in $\Omega(\Gamma)$. There is a short transversal $\tau$ of $F(\xi)$ such that $|\gamma \cap \tau| \geq 2$ and there are leaves of $F(\xi)$ passing through $\Gamma$ which intersect $\tau$ between two points $p_{1}, p_{2}$ of $\gamma \cap \tau$. Because $\gamma$ is recurrent it cannot intersect $\Gamma$. Let $I \subset \tau$ be the maximal open segment lying between $p_{1}, p_{2}$ such that the leaves of $F(\xi)$ induce a map from $I$ to $\Gamma$. It follows (as in Proposition 14.1.4 in [23]) that the boundary points of $I$ connect to singular points of $F(\xi)$ which have to be hyperbolic by our assumptions. These hyperbolic singularities are part of a path tangent to $F(\xi)$ which connects $\Gamma$ with $\tau$ and this path passes only through hyperbolic singularities. This is a contradiction to our assumption $\Gamma \cap P_{0}=\varnothing$. Thus if $P_{0} \cap \Gamma=\varnothing$, then there are two cases depending on the nature of $\Omega(\Gamma)$.

If $\Omega(\Gamma)$ is an elliptic singularity $x$, respectively a closed leaf $\gamma$ of $F(\xi)$, then we place no vertices on $\Gamma$ and $\alpha$ maps $\Gamma$ to the elliptic point, respectively the closed leaf while $\alpha=\alpha_{0}$ outside a collar of $\Gamma$.

In order to fix $\alpha$ on a collar of $\Gamma$ we choose a collar $\Gamma \times[-1,0]$ of $\Gamma=\Gamma \times\{0\}$ such that $\alpha_{0}(\Gamma \times\{t\})$ is transverse to $F(\xi)$ for all $t \in[-1,0]$.

Now choose a disc $D_{x}$ around $x$ respectively a collar $U_{\gamma} \simeq \gamma \times(-1 / 2,0]$ of $\gamma$. On $\Gamma \times(-1,0]$ choose $\alpha$ such that $\{y\} \times(-1,-1 / 2]$ is mapped to the segment of a leaf 
of $F(\xi)$ which connects $y \times\{-1\}$ to $\partial D_{x}$ or $\gamma \times\{-1 / 2\}$. On $D_{x}$ respectively $U_{\gamma}$ we choose a foliation without closed leaves which coincides with $F(\xi)$ near $\partial D_{x}$ respectively $\gamma \times\{-1 / 2\}$ and which coincides with the foliation by radial lines near $x \in D_{x}$ respectively the foliation induced by the second factor of $\gamma \times(-1 / 2,0]$ near $\gamma=\gamma \times\{0\}$. Now we extend the map $\alpha$ from $\Gamma \times(-1,1 / 2]$ to $\Gamma \times(-1,0]$ such that the foliation on $\Gamma \times[-1 / 2,0]$ gets mapped to the chosen foliation on the disc or on $\gamma \times(-1 / 2,0]$.

If $\Omega(\Gamma)$ is a cycle containing hyperbolic points, then we place a corner on $\Gamma$ for each time the cycle passes through a hyperbolic singularity. On a collar of $\Gamma$, the map $\alpha$ is defined in a similar fashion as in the previous case.

Next, we consider a boundary component $\Gamma$ of $Q_{0}$ which is transverse to $F(\xi)$ and contains an element $p$ of $P_{0} \cap \Gamma$. Let $\eta$ be an unstable leaf of the corresponding hyperbolic singularity $\alpha(p)$ of $F(\xi)$ and $\Omega(\eta)$ the $\omega$-limit set of $\eta$. Depending on the type of $\Omega(\eta)$ we distinguish four cases.

(i) $\Omega(\eta)$ is an elliptic singular point $x$. Then we place a vertex on $\Gamma$ next to the pseudovertex unless $x$ already appeared in the construction and $\alpha$ maps all edges on $\Gamma$ to unstable leaves of hyperbolic singularities. The edge between the pseudovertex and the new vertex is mapped to $\eta$ by $\alpha$.

(ii) $\Omega(\eta)$ is a cycle of $F(\xi)$ or a quasi-minimal set. Then we place a vertex $v$ on $\Gamma$ and add this vertex to the set of virtual vertices $V_{0}$.

(iii) $\Omega(\eta)$ is a hyperbolic point and $\alpha(p)$ is part of a cycle. Some possible configurations in this case are shown in Figure 3 (except the top right part). More precisely, the configurations in Figure 3 correspond to the case when there are at most two different hyperbolic singularities of $F(\xi)$ which are connected. (This assumption is satisfied for surfaces in a generic 1-parameter family of embeddings.)

We add a 1 -handle to $Q_{0}$ along $\Gamma$. This defines a new polygon $Q_{1}$. We define $\alpha_{1}: Q_{1} \rightarrow F$ such that one of two new boundary components is mapped to the cycle containing $\alpha(p)$ and we place a corner on this connected component of $\partial Q_{1}$ for each time the cycle passes trough a hyperbolic singularity. In particular, $p$ is no longer a pseudovertex. Outside of a collar of $\Gamma$ we require $\alpha_{1}=\alpha_{0}$.

(iv) $\Omega(\eta)$ is a hyperbolic singularity and $\alpha(p)$ is not part of a cycle. Then we place a corner on $\Gamma$ which corresponds to $\Omega(\eta)$. We continue with the unstable leaf $\eta^{\prime} \subset \overline{B_{\omega}(\Gamma)}$ of $\Omega(\eta)$ and place corners or vertices on $\Gamma$ depending on the nature of the $\omega$-limit set of $\eta^{\prime}$. One possible configuration is shown in the top right part of Figure 3.

All unstable leaves of hyperbolic singularities in $F(\xi)$ which correspond to elements of $P_{0} \cap \Gamma$ can be treated in this way. 

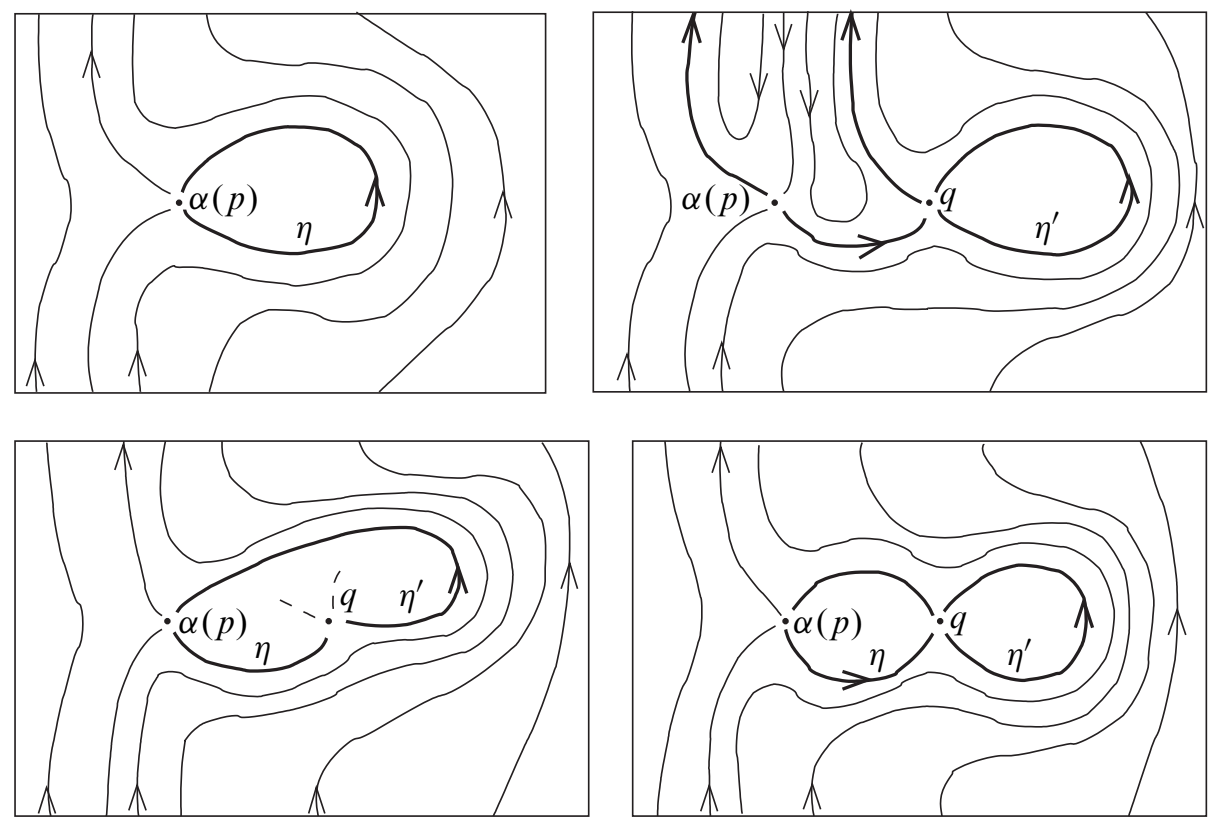

Figure 3

We iterate the procedure (starting from the choice of pseudovertices) until no new 1handles are added and we have treated all occurring boundary components and unstable leaves of pseudovertices. This process is finite because each hyperbolic singularity can induce the addition of at most one 1-handle and there are only finitely many hyperbolic singularities on $F$. Once it is determined how boundary components of $Q$ are mapped to leaves of the characteristic foliation on $F$ one obtains the embedding $\alpha$ using $F(\xi)$ in a similar way as in the case when $P_{0}=\varnothing$ above. In the end we obtain the desired Legendrian polygon $(Q, V, \alpha)$.

\subsection{The elimination lemma}

There are several possibilities to manipulate the characteristic foliation on an embedded surface. Of course one can always perturb the embedding of the surface so that it becomes generic and that the singularities lie in the interior of the contact region $H(\xi)$ or in the interior of its complement. In addition to such perturbations we shall use two other methods.

The first method discussed in this section is called elimination of singularities and it is well known in the context of contact structures. The second method will be described in Section 3.4. 
By a $C^{0}$-small isotopy of the surface $F$ one can remove a hyperbolic and an elliptic singularity which are connected by a leaf $\gamma$ of $F(\xi)$ if the signs of the singularities agree. The characteristic foliation before the isotopy is depicted in Figure 4. The segment $\gamma$ corresponds to the thickened segment in the middle.

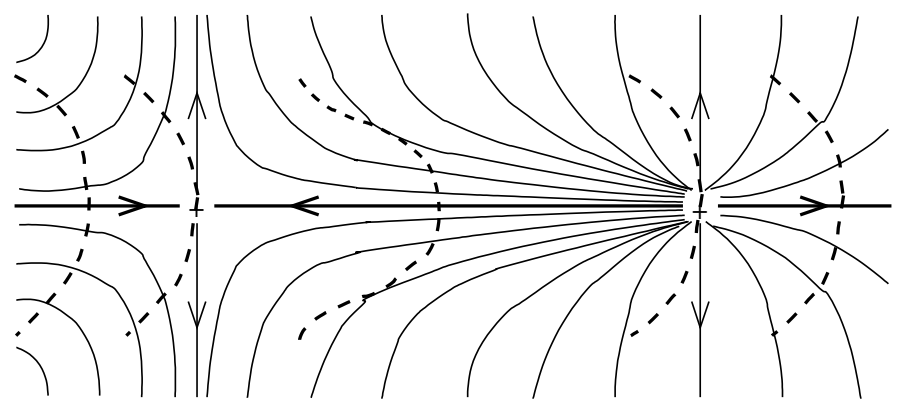

Figure 4

After the isotopy the characteristic foliation on a neighbourhood of $\gamma$ looks like in Figure 5. The elimination of singularities plays an important role in Eliashberg's proof

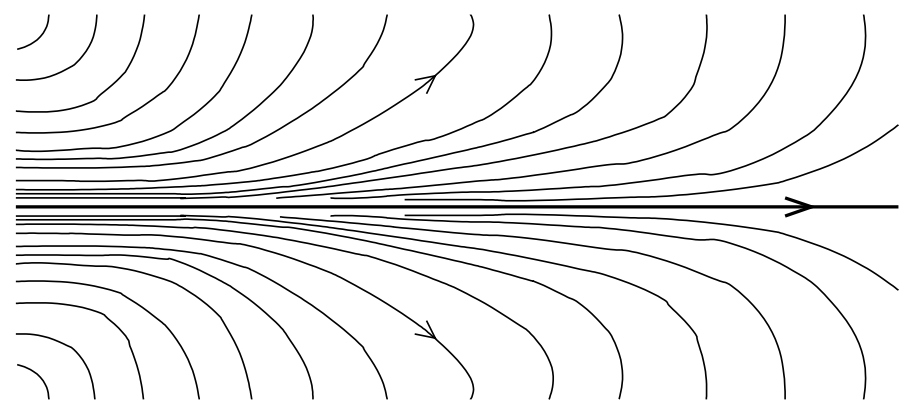

Figure 5

of the Thurston-Bennequin inequalities (Theorem 1.6) for tight contact structures.

Below we give a proof of the elimination lemma which applies to confoliations under a condition on the location of the singularities. Usually (see Aebischer et al [1] and Geiges [15] for example) the elimination lemma is proved using Gray's theorem but this theorem is not available in the current setting.

Lemma 3.5 Let $F$ be a surface in a confoliated manifold $(M, \xi)$. Assume that the characteristic foliation on $F$ has one hyperbolic singularity and one elliptic singularity of the same sign which are connected by a leaf $\gamma$ of the characteristic foliation. 
If the elliptic singularity lies in $H(\xi)$, then there is a $C^{0}$-small isotopy of $F$ with support in a small open neighborhood $U$ of $\gamma$ such that the new characteristic foliation has no singularities inside of $U$. The isotopy can be chosen such that $\gamma$ is contained in the isotoped surface.

Proof We assume that both singularities are positive. There is a neighbourhood $U$ of $\gamma$ with coordinates $x, y, z$ such that $\left.\xi\right|_{U}$ is defined by the 1 -form $\alpha=d z+a(x, y, z) d y$ where the function $a$ satisfies $\partial_{x} a \geq 0$. We assume that $\partial_{z}$ is positively transverse to $\xi$ and $F,\{z=0\} \subset F$ and the $x$-axis of the coordinate system contains $\gamma$.

Let $U^{\prime} \subset U$ be diffeomorphic to a closed ball such that $\partial_{x}$ is tangent to $\partial U^{\prime}$ along a circle and $U^{\prime}$ contains $\gamma$ in its interior. We extend $\left.\xi\right|_{U^{\prime}}$ to a complete connection $\xi_{c}$ on $\mathbb{R}^{3}$ by extending $a$ to a function on $\mathbb{R}^{3}$ satisfying $\partial_{x} a \geq 0$ and $a(x, y, z)$ is constant for $|z|>C$ where $C>\sup \left\{z \mid(x, y, z) \in U^{\prime}\right\}$. Note that by our assumption on $U^{\prime}$ we may assume that intersection of $\partial_{x}$ with $U^{\prime}$ is connected (or empty). The requirement that $a$ is constant when $|z|$ is large ensures that $\xi_{c}$ is a complete connection.

We choose $\varepsilon>0$ so that $p \times(-\varepsilon, \varepsilon) \subset U^{\prime}$ (the second factor corresponds to the $z$-coordinate) for all $p$ in a neighbourhood $V \subset F$ of $\gamma$. Since every step in the proof will take place in a fixed small neighbourhood of $\gamma$ we can apply Lemma 2.1 without any restriction.

For a path $\sigma \subset V$ we will consider the hypersurface $T_{\sigma}=\sigma \times(-\varepsilon, \varepsilon)$. By our choices $T_{\sigma}(\xi)$ is transverse to the second factor of $T_{\sigma}$.

Choose a smooth foliation $\mathcal{I}$ of a small neighbourhood (contained in $V$ ) of $\gamma$ in $F$ by intervals $I_{s}, s \in[-1,1]$ as indicated by the dashed lines in Figure 4. We require $\mathcal{I}$ to have the following properties.

(i) Two intervals $I_{s_{0}}, I_{s_{1}}$ pass through the singularities. One of them is tangent to the closure of the unstable separatrices of the hyperbolic singularity.

(ii) All intervals intersecting the interior of $\gamma$ have exactly two tangencies with the characteristic foliation on $F$. The intervals which do not intersect the closure of $\gamma$ are transverse to the characteristic foliation.

(iii) Let $\sigma$ by a path in $F$ which is shorter than $\delta$ with respect to a fixed auxiliary Riemannian metric. If $\delta>0$ is small enough, then the image of $(\sigma(0), 0)$ under the holonomy along $T_{\sigma}$ is defined. We assume that the length of each $I_{S}$ is smaller than $\delta$.

We parameterize the leaf $I_{S}$ by $\sigma_{s}:[0,1] \rightarrow F$ such that the intersection of $\gamma$ with $I_{S}$ is positive (or empty), ie in Figure 4 the leaves of $\mathcal{I}$ are oriented towards the upper part of the picture. 
The following figures show neighbourhoods of $I_{s}$ in $T_{s}:=T_{\sigma_{s}}$ for certain $s \in[-1,1]$. In each of these figures the dashed line represents $I_{s}$, it is oriented from left to right. Figure 6 corresponds to a leaf $I_{s}$ which does not intersect $\gamma$. Then $I_{s}$ is nowhere tangent to the characteristic foliation on $T_{s}$. By our orientation conventions and the choice of $\mathcal{I}$ the slope of $\xi \cap T_{S}$ is negative along $I_{S}$.

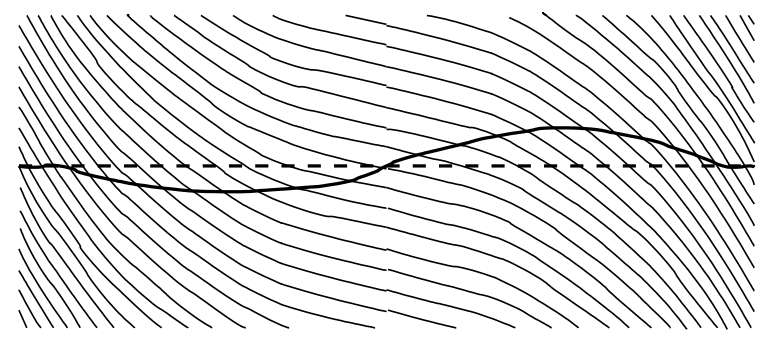

Figure 6

The leaves $I_{s_{0}}, I_{s_{1}}$ contain the singular points of the characteristic foliation on $F$. As shown in Figure 7 there is exactly one tangency of $F$ and the characteristic foliation on $T_{s_{0}}, T_{s_{1}}$. The slope of the characteristic foliation on $T_{s_{0}}, T_{s_{1}}$ is negative along $I_{s_{0}}, I_{s_{1}}$ except at the point of tangency.

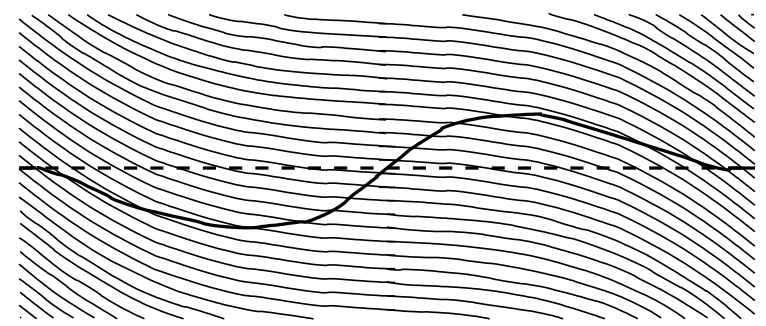

Figure 7

Finally, the leaves $I_{s}, s \in\left(s_{0}, s_{1}\right)$ intersect the interior of $\gamma$ and $I_{s}$ is tangent to $F(\xi)$ in exactly two points. This is shown in Figure 8. Between the two points of tangency, the slope of the characteristic foliation on $T_{s}$ is positive along $I_{s}$, it is zero at the tangencies and negative at the remaining points of $I_{S}$.

We want to find a smooth family of isotopies of the intervals $I_{S}$ within $T_{S}$ such that

(i) for all $s$ the isotopy is constant near the endpoints of $I_{S}$ and

(ii) after the isotopy, the intervals $I_{S}$ are transverse to the characteristic foliation on $T_{s}$. 


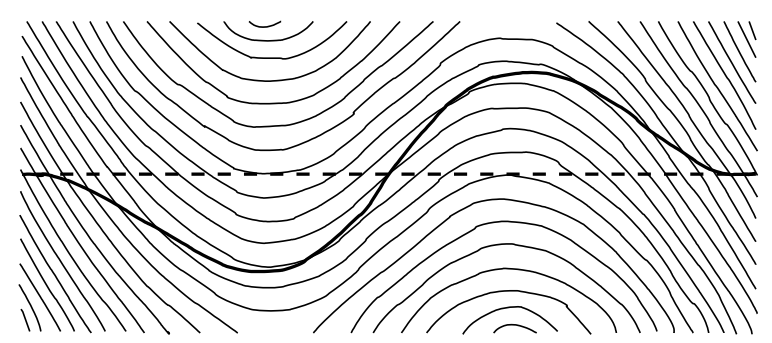

Figure 8

This will produce the desired isotopy of $F$. Such a family of isotopies exists if and only if the following condition $\mathrm{C}(\mathrm{s})$ is satisfied for all $s \in[-1,1]$.

Condition C(s) The image of $\sigma_{s}(0) \times\{0\}$ under the holonomy along $\sigma_{s}$ lies below the other endpoint $\sigma_{s}(1) \times\{0\}$ of $I_{s}$ or the leaf of $T_{s}(\xi)$ which passes through $\left(\sigma_{s}(0), 0\right)$ exits $T_{s}$ through $\left(\sigma_{s},-\varepsilon\right) \subset \partial T_{s}$.

Note that this condition is automatically satisfied for $s \in[-1,1]$ if $I_{S}$ does not intersect $\gamma$ or this intersection point is close enough to a singularity of the characteristic foliation. If $\mathrm{C}(\mathrm{s})$ is not satisfied for all $s$, then we will replace $\mathcal{I}$ by another foliation $\mathcal{I}^{\prime}$ by intervals $I_{s}^{\prime}$ (the corresponding embeddings of intervals are denoted by $\sigma_{s}^{\prime}$ ) as follows:

(i) If $I_{S}$ does not intersect $\gamma$, then $\sigma_{s}=\sigma_{s}^{\prime} . I_{s}^{\prime}$ intersects $\gamma$ if and only if $I_{s}$ does.

(ii) $I_{s}^{\prime}$ is tangent to the characteristic foliation on $F$ along two closed intervals (which may be empty or points). The complement of these two intervals is the union of three intervals such that each of these intervals is mapped to a curve of length $\leq \delta$.

(iii) $I_{S}$ and $I_{s}^{\prime}$ coincide on those intervals where the characteristic foliation on $T_{S}$ has negative slope for all $s \in[-1,1]$.

(iv) $\bar{I}_{S} \cup I_{S}^{\prime}$ bounds a positively oriented disc (here $\bar{I}_{S}$ denotes the interval $I_{s}$ with the opposite orientation).

In Figure 9 the dashed line corresponds to $I_{s}^{\prime}$ while the thick solid line represents $I_{S}$. For $s \in\left(s_{0}, s_{1}\right)$ we define a curve $I_{s}^{\prime \prime}$ by replacing the segment of $I_{s}$ lying between the tangencies with $F(\xi)$ by two segments of leaves of $F(\xi)$ whose $\alpha$-limit set is the elliptic singularity in $V$. Then the holonomy on $I_{s}^{\prime \prime} \times(-\varepsilon, \varepsilon)$ satisfies the condition $\mathrm{C}(\mathrm{s})$ : The leaf of the characteristic foliation on $I_{s}^{\prime \prime} \times(-\varepsilon, \varepsilon)$ starting at $\sigma_{s}(0) \times 0$ can never return to $I_{s}^{\prime \prime}$ since at the starting point of the leaf, the slope of the leaf is negative 


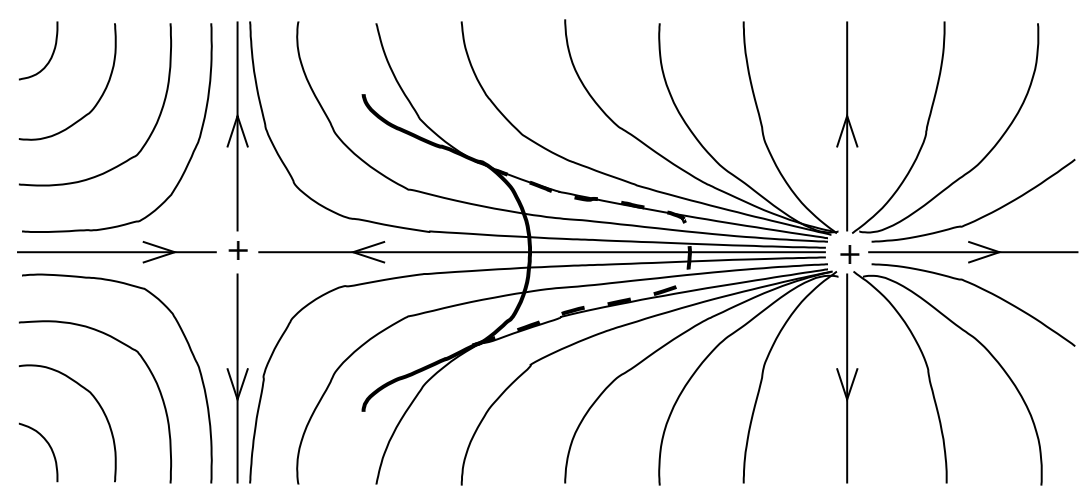

Figure 9

while it is nonnegative along $I_{s}^{\prime \prime}$. This shows that for each $s$ one can choose $I_{s}^{\prime}$ with the desired properties.

Whenever $I_{s}$ satisfies $\mathrm{C}(\mathrm{s})$ then so does $I_{s}^{\prime}$ by Lemma 2.1. It follows that we can choose the foliation $\mathcal{I}^{\prime}$ such the leaf $I_{s}^{\prime}$ of $\mathcal{I}^{\prime}$ satisfies $\mathrm{C}(\mathrm{s})$ for all $s \in[-1,1]$. The desired isotopy of $F$ can be constructed such that the surface is transversal to $\partial_{z}$ throughout the isotopy.

The following lemma is a partial converse of the elimination lemma. Because it is only concerned with the region where $\xi$ is a contact structure we omit the proof. It can be found in $[9 ; 16]$.

Lemma 3.6 Let $F \subset M$ be an embedded surface in a confoliated manifold and $\gamma \subset F$ a compact segment of a nonsingular leaf of the characteristic foliation on $F$ which lies in the contact region of $\xi$.

Then there is a $C^{0}$-small isotopy of $F$ with support in a little neighbourhood of $\gamma$ such that after the isotopy there is an additional pair of singularities (one hyperbolic and one elliptic) having the same sign. The isotopy can be performed in such a way that $\gamma$ is still tangent to the characteristic foliation and connects the two new singularities.

We end this section with mentioning a particular perturbation of an embedded surface $F$ which also appears in [9]. Consider an injective Legendrian polygon $(Q, V, \alpha)$ such that there is an elliptic singularity $x$ of $F(\xi)$ such that $\alpha^{-1}(x)$ consists of more than one vertex of $Q$. Since $x$ appears as the limit set of leaves of the characteristic foliation, $x$ cannot lie in the interior of the foliated region and after a small perturbation we may assume $x \in H(\xi)$. 
Then $F$ can be deformed by a $C^{0}$-small isotopy near $x$ into a surface $F^{\prime}$ such that there is a map $\alpha^{\prime}: Q \rightarrow F^{\prime}$ with the same properties as $\alpha$ which coincides with $\alpha$ outside a neighbourhood of $\alpha^{-1}(x)$ and $\alpha^{\prime}$ maps all vertices in $\alpha^{-1}(x)$ to different elliptic singularities of $F^{\prime}(\xi)$; see Figure 10 .

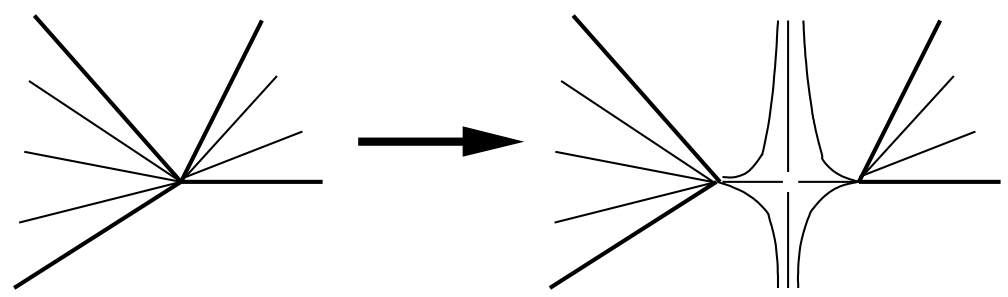

Figure 10

\subsection{Modifications in the neighbourhood of integral discs}

The second method for the manipulation of the characteristic foliation on an embedded surface $F$ is by surgery of the surface along a cycle $\gamma$ which is part of an integral disc of $\xi$. The latter condition is satisfied when the confoliation is tight and $\gamma$ bounds a disc in $F$ (for example when $F$ is simply connected).

While the elimination lemma is used to prove the Thurston-Bennequin inequalities for tight contact manifolds, the following lemmas adapt lemmas appearing in Roussarie [29] and Thurston [31] (cf also Candel and Conlon [5]) from the proof of the existence of the Roussarie-Thurston normal form for surfaces in 3-manifolds carrying a foliation without Reeb components. The existence of this normal form implies the ThurstonBennequin inequalities for such foliations.

Lemma 3.7 Let $F$ be a surface and $\gamma$ a closed leaf of the characteristic foliation on $F$ such that there is a disc $D$ tangent to $\xi$ which bounds $\gamma$ and $F \cap D=\gamma$.

Then there is a surface $F^{\prime}$ which is obtained from $F$ by removing an annulus around $\gamma$ and gluing in two discs $D_{+}, D_{-}$contained in a tubular neighbourhood of $D$. The discs can be chosen such that $D_{+}(\xi), D_{-}(\xi)$ have exactly one elliptic singularity in their interior and these singularities have opposite signs.

If the germ of the holonomy of $h_{\partial D}$ is non trivial along $\gamma$ on one side of $\gamma$, then we can arrange that the elliptic singularity on the disc on that side lies in the interior of the contact region and every leaf of the characteristic foliation on the new discs connects the singularity with the boundary of the disc. 
Proof We will construct $D_{+}$in the presence of nontrivial holonomy on the upper side of $\gamma \subset F$. The construction of the other disc is analogous.

Fix a closed neighbourhood $U \simeq D \times(-\varepsilon, \varepsilon), \varepsilon>0$ of $D$ such that the fibers of $D \times(-\varepsilon, \varepsilon) \rightarrow D$ are positively transverse to $\xi$. We assume $F \cap U=\partial D \times(-\varepsilon, \varepsilon)$. By Lemma 2.1 there is a point $(x, \eta) \in D \times(0, \varepsilon)$ lying in the contact region and we identify $D \times\{0\}$ with the unit disc in $\mathbb{R}^{2}$ such that $x$ corresponds to the center.

On $D$ we consider the singular foliation consisting of straight lines starting at the center. For $t \in[-\varepsilon, \varepsilon]$ let $D_{t}$ be the disc formed by horizontal lifts of leaves of the singular foliation on $D$ with initial point $(x, t)$. By construction, $D_{\eta}(\xi)$ is diffeomorphic to the singular foliation by radial lines on a neighbourhood of $(x, \eta) \in D_{\eta}$ and the zero $(x, \eta)$ of the restriction of a defining form $\alpha$ of $\xi$ is nondegenerate since $(x, \eta)$ lies in the contact region. We assume that $D_{t}(\xi)$ has no other singularity lying above the disc of radius $1 / 2$ (otherwise we choose a smaller radius in the sequel).

Let $\rho:[\eta, \varepsilon] \rightarrow[1 / 2,1]$ be a monotone function which is smooth on $(\eta, \varepsilon]$ such that $\rho \equiv 1$ near $\varepsilon$ and the graph of $\rho$ is $C^{\infty}$-tangent to a vertical line at $(\eta, 1 / 2)$. We denote the boundary of the disc of radius $\rho(t)$ in $D_{t}$ by $S_{t}$. The union of all $S_{t}, t \in\left[\eta, \eta^{\prime}\right]$, with the part of $D_{\eta}$ which corresponds to the disc with radius $1 / 2$ is the desired disc $D_{+}$. We remove the annulus $\partial D \times\left[0, \eta^{\prime}\right]$ from $F$ and add $D_{+}$.

By construction the only singular point of $D_{+}(\xi)$ is $(x, \eta)$, the singularity is elliptic and contained in the contact region.

In order to show that all leaves of $D_{+}(\xi)$ accumulate at the elliptic singularity it is enough to show that there are no closed leaves on $D_{+}$. Assume that $\tau$ is a closed leaf of $D_{+}(\xi)$. Let $D_{\tau}$ be the disc formed by lifts of the leaves of the radial foliation on $D$ with initial point on $\tau$.

The restriction of $\xi$ to $D \times[0, \varepsilon]$ extends to a confoliation $\tilde{\xi}$ on $\mathbb{R}^{2} \times \mathbb{R}$ which is a complete connection. By Proposition $2.5 \widetilde{\xi}$ is tight. Hence $\tau$ must bound an integral disc of $\xi^{\prime}$. Now $D_{\tau}$ is the only possible candidate for such a disc. But $D_{\tau}$ cannot be an integral disc of $\widetilde{\xi}$ because it intersects the contact region of $\widetilde{\xi}$ (or equivalently $\xi$ ) in an open set. This contradiction finishes the proof.

The following two lemmas are analogues to the elimination lemma in the sense that we will remove pairs of singularities. However, new singularities can be introduced: In Lemma 3.9 we will obtain a surface whose characteristic foliation is not generic. However this will play no role in later applications since the locus of the nongeneric singularities will be isolated by closed leaves of the characteristic foliation and these singularities do not contribute to $e(\xi)[F]$ or $\chi(F)$. 
Lemma 3.8 Let $F$ be a surface in a confoliated manifold, $D$ an embedded disc tangent to $\xi$ and $D \cap F=\gamma$ a cycle containing exactly one corner $x_{0}$.

Then there is a surface $F^{\prime}$ which is obtained from $F$ by removing a tubular neighbourhood of $\gamma$ and gluing in two discs $D_{+}, D_{-}$. The characteristic foliation of $F^{\prime}$ has no singularities on $D_{-}$and one elliptic singularity on $D_{+}$whose sign is the opposite of the sign of $x_{0}$.

Proof The assumptions of the lemma imply that $x_{0}$ has a stable and an unstable leaf which do not lie on $D$. Fix a product neighbourhood $U \simeq \widetilde{D} \times(-\varepsilon, \varepsilon)$ of $D$ with the following properties.

(i) $D$ is contained in the interior of the disc $\widetilde{D} \times\{0\}$.

(ii) There is a simple Legendrian curve $\sigma \subset \widetilde{D}$ containing $x_{0}$ in its interior and intersecting $\partial D$ in two points and $\partial \widetilde{D}$ in two points such that $\gamma$ is nowhere tangent to $\sigma$ and $\partial \widetilde{D}$ is transverse to $\sigma$. The points in $\gamma \cap \sigma$ are denoted by $x_{0}, x_{1}$.

(iii) The fibers of $\pi: \widetilde{D} \times(-\varepsilon, \varepsilon) \rightarrow \widetilde{D}$ are transverse to $\xi$ and $F$.

Now consider $T_{\sigma}=\sigma \times(-\varepsilon, \varepsilon)$. The intersection $T_{\sigma} \cap F$ has a nondegenerate tangency with $T_{\sigma}(\xi)$ in $x_{0}$ and meets $\sigma \times\{0\}$ transversely in $x_{1}$. We choose two points $y_{0}, y_{1} \in T_{\sigma} \cap F$ such that $x_{0}$ lies between $\pi\left(y_{0}\right)$ and $x_{1}$ and $y_{1}$ lies on the other side of $\widetilde{D}$ (as indicated in Figure 11).

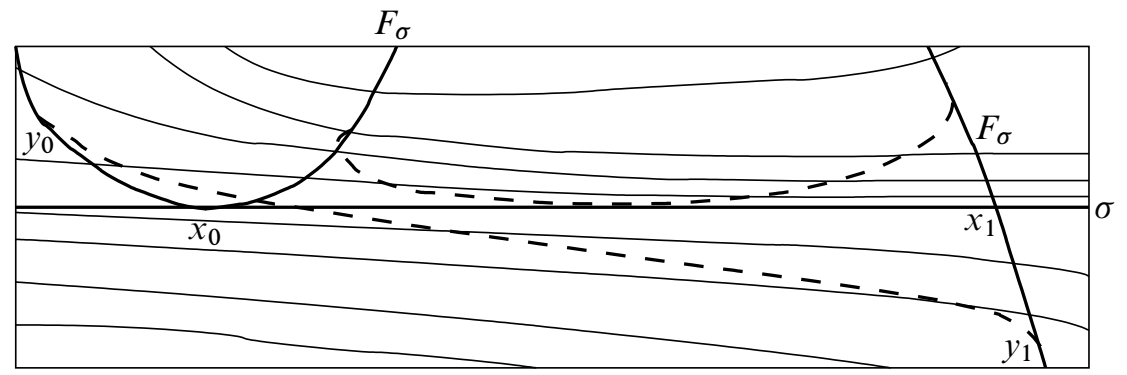

Figure 11

The points $y_{0}, y_{1}$ can be connected by a curve $\hat{\sigma} \subset T_{\sigma}$ transverse to the characteristic foliation on $T_{\sigma}$ provided that $y_{0}, y_{1}$ are close enough to $\widetilde{D}$. Moreover, we may assume that $\widehat{\sigma}$ is tangent to $F$ near its endpoints (cf the lower dashed curve in Figure 11).

The curve $\hat{\sigma}$ is going to be part of $D_{-}$. In order to finish the construction of $D_{-}$we choose a foliation of $\widetilde{D}$ by a family $I_{s}, s \in \sigma$ of intervals that connect boundary points 
of $\widetilde{D}$ and are transverse to $\sigma$. The characteristic foliation on $T_{I_{s}}$ consists of lines which are mapped diffeomorphically to $I_{S}$ by $\pi$.

If $\hat{\sigma}$ was chosen close enough to $\widetilde{D}$, then there is a smooth family of curves $\hat{I}_{S} \subset$ $I_{s} \times(-\varepsilon, \varepsilon), s \in \sigma$ such that $\widehat{I}_{s}$

(i) intersects $\hat{\sigma}$ exactly once and is tangent to $\xi$ in this point,

(ii) is transverse to $\xi$ elsewhere and

(iii) is tangent to $F$ near $y_{0}, y_{1}$.

Note that the characteristic foliation on $I_{S} \times(-\varepsilon, \varepsilon)$ is a smooth family of lines which are nearly parallel to $I_{S}$ if $\varepsilon>0$ is small enough and $F$ is transverse to all these lines except at the hyperbolic singularity.

The choices we made for $\hat{\sigma}$ and $\widehat{I}_{s}, s \in \sigma$ ensure that the union of all curves $\widehat{I}_{s}$ is a disc $D_{-}$which is transverse to $\xi$. The disc $D_{+}$is obtained as in the proof of Lemma 3.7. The statement about the sign of the singularity of $D_{+}(\xi)$ follows from the construction.

Lemma 3.9 Let $F \subset M$ be an embedded surface in a manifold carrying a confoliation $\xi$ such that $F(\xi)$ contains a hyperbolic singularity $x$ and the stable and unstable leaves of $x$ bound an annulus $A \subset F$ which is pinched at $x$. We assume that the pinched annulus is bounded by an integral disc $D$ of $\xi$ such that $\partial A=F \cap D$.

Then there is an embedded surface $F^{\prime}$ which is obtained from $F$ by removing a neighbourhood of $\gamma$ and gluing in an annulus $A^{\prime}$ and a disc $D^{\prime}$ such that $A^{\prime}(\xi)$ has one of the following properties.

(i) $A^{\prime}(\xi)$ has no singularity.

(ii) The singularities of $A^{\prime}(\xi)$ form a circle and a neighbourhood in $F^{\prime}$ of this circle is foliated by closed leaves of $F^{\prime}(\xi)$.

The characteristic foliation on $D^{\prime}$ has exactly one singularity which is elliptic and whose sign is opposite to the sign of $x$.

Proof The disc $D$ in the assumptions of the lemma is an immersed disc which is an embedding away from two points in the boundary. These two points are identified to the single point $x$. Let $S^{1} \simeq \sigma \subset D$ be a simple closed curve in $D$ which meets $x$ exactly once.

We choose a solid torus $C=\sigma \times[-1,1] \times[-1,1]$ such that $\sigma=\sigma \times\{(0,0)\}$ and the foliation corresponding to the second factor is Legendrian while the foliation 
corresponding to the third factor is transverse to $\xi$. For $s \in[-1,1]$ let $A_{s}=\sigma \times$ $\{s\} \times[-1,1]$. The torus is chosen such that $D \subset \sigma \times[-1,1] \times\{0\}$ and $F$ intersects $A_{-}=\sigma \times[-1,1] \times\{-1\}$ in two circles bounding an annulus while $F \cap(\sigma \times[-1,1] \times\{1\})$ is a circle which bounds is disc in $\sigma \times[-1,1] \times\{1\}$.

A disc $D^{\prime}$ which bounds $F \cap(\sigma \times[-1,1] \times\{1\})$ with the desired properties can be constructed as in the proof of Lemma 3.7.

Let $P_{S}:=\sigma(s) \times[-1,1] \times[-1,0], s \in S^{1}$. The characteristic foliation on $P_{S}$ consists of lines transverse to the last factor of $P_{S}$ and $\sigma(s) \times[-1,1] \times\{0\}$ is a leaf of $P_{S}(\xi)$

If $\xi$ one of the annuli $\sigma \times\{t\} \times(-1,0], t \in(-1,1)$ has nontrivial holonomy along $\sigma \times\{(t, 0)\}$ or if $\sigma \times\{(t, 0)\}$ is not Legendrian, then one can choose a curve $\sigma^{\prime}$ in that annulus which is transverse to $\xi$. The annulus $A^{\prime}$ is the union of curves in $P_{s}, s \in S^{1}$ which connect the two points of $F \cap\left(\sigma(s) \times[-1,1] \times\{-1\}\right.$ and pass through $\sigma^{\prime} \cap P_{S}$. These curves can be chosen such that they are transverse to $P_{S}(\xi)$ everywhere except in $\sigma^{\prime} \cap P_{S}$. By construction $A^{\prime}(\xi)$ has the property described in (i) of the lemma.

This construction also applies if we choose $\sigma^{\prime}$ in annuli which are $C^{\infty}$-close to $\sigma \times\{t\} \times[-1,0]$ for a suitable $t \in[-1,1]$. If all annuli of this type have trivial holonomy along their boundary curve which is close to $\sigma \times\{(t, 0)\}$, then $\xi$ is a foliation on a neighbourhood of $\sigma$ in $\sigma \times[-1,1] \times[-1,0]$ by Lemma 2.1. The holonomy along $\sigma$ is trivial. Choosing $\sigma^{\prime}$ tangent to a leaf, the same construction as in the previous case (with $\sigma^{\prime}=\sigma$ ) yields an annulus $A^{\prime}$ with the properties described in (ii).

Lemma 3.7 and Lemma 3.8 suffice for Section 4 because the embedded surfaces in that section are going to be simply connected.

In the lemmas of this section we have assumed that $F \cap D=\gamma$. In general $F$ and $D$ may intersect elsewhere. Since all singularities of the characteristic foliation on $\gamma$ are nondegenerate or of birth-death type, there is a neighbourhood of $\gamma$ in $D$ such that $\gamma$ is the intersection of $F$ with this neighbourhood. After a small perturbation with support outside of a neighbourhood of $\gamma$ we may assume that $F$ is transverse to $D$ on the interior of $D$. Now we can apply Lemma 3.7 a finite number of times to circles in $F \cap D$ in order to achieve that the resulting surface intersects $D$ only along $\gamma$. Then we can apply the lemmas of this section.

\section{Rigidity results for tight confoliations}

In this section we establish some restrictions on the homotopy class of plane fields which contain tight confoliations. One of them is Thurston-Bennequin inequality for 
simply connected surfaces. Note that this imposes no restriction on the Euler class $e(\xi)$ of a tight confoliation $\xi$ on a closed manifold $M$ unless the prime decomposition of $M$ contains $\left(S^{1} \times S^{2}\right)$-summands. Further restrictions on the homotopy class of $\xi$ follow from:

Theorem 4.1 Let $M$ be a manifold carrying a tight confoliation $\xi$ and $B \subset M$ a closed embedded ball in $M$. There is a neighbourhood of $\xi$ in the space of plane fields with the $C^{0}$-topology such that $\left.\xi^{\prime}\right|_{B}$ is tight for every contact structure $\xi^{\prime}$ in this neighbourhood.

The proof of this theorem is given in Section 4.2. Let us explain an application of Theorem 4.1 which justifies the claim that Theorem 4.1 is a rigidity statement about tight confoliations.

By Theorem 1.1 every confoliation on a closed manifold can be $C^{0}$-approximated by a contact structure unless it is a foliation by spheres. Hence Theorem 4.1 can be applied to every confoliation. Recall the following theorem.

Theorem 4.2 (Eliashberg [9]) Two tight contact structures on the 3-ball $B$ which coincide on $\partial B$ are isotopic relative to $\partial B$.

It follows from this theorem that two tight contact structures on $S^{3}$ are isotopic and therefore homotopic as plane fields. In contrast to this every homotopy class of plane fields on $S^{3}$ contains a contact structure which is not tight. Thus the following consequence of Theorem 4.1 shows that there are restrictions on the homotopy classes of plane fields containing tight confoliations.

Corollary 4.3 Only one homotopy class of plane fields on $S^{3}$ contains a positive tight confoliation.

Proof Let $\xi$ be a tight confoliation on $S^{3}$. It is well known that every foliation of rank 2 on $S^{3}$ contains a Reeb component; see Novikov [27]. Thus $H(\xi)$ is not empty. We choose $p \in H(\xi)$ and a ball $B \subset H(\xi)$ around $p$.

According to [10] $\xi$ can be $C^{0}$-approximated by a contact structure $\xi^{\prime}$ on $S^{3}$ such that $\xi$ and $\xi^{\prime}$ coincide on $B$. By Theorem 4.1 the restriction of $\xi^{\prime}$ to $S^{3} \backslash B$ is tight and by a result from [7] $\xi^{\prime}$ is a tight contact structure on $S^{3}$ which is homotopic to $\xi$.

More generally, Theorem 4.1 together with Theorem 4.2 implies that the homotopy class of a tight confoliation $\xi$ as a plane field is completely determined by the restriction of $\xi$ to a neighbourhood of the 2 -skeleton of a triangulation of the underlying manifold (here we fix the distribution $\xi$ and not the homotopy class of $\xi$ as a plane field near the 2-skeleton). 


\subsection{The Thurston-Bennequin inequality for discs and spheres}

In this section we prove the Thurston-Bennequin inequalities for a tight confoliation $\xi$ in the cases where $F$ is a sphere or a disc (with transverse boundary). For this we adapt the arguments in [9]. We shall discuss why Eliashberg's proof cannot be adapted for nonsimply connected surfaces in tight confoliations after the proof Theorem 4.4. Recall that the self-linking number $\operatorname{sl}(\gamma, F)$ of a null-homologous knot $\gamma$ which is positively transverse to $\xi$ with respect to a Seifert surface $F$ satisfies $e(\xi)[F]=-\operatorname{sl}(\gamma, F)$ where $e(\xi)[F]$ corresponds to the obstruction for the extension of the characteristic foliation near $\partial F$ to a trivialization of $\left.\xi\right|_{F}$.

Theorem 4.4 Let $(M, \xi)$ be a manifold with a tight confoliation. Then

(a) $e(\xi)\left[S^{2}\right]=0$ for every embedded $2-$ sphere $S^{2} \subset M$ and

(b) $\operatorname{sl}(\partial D, D) \leq-1$ for every embedded disc whose boundary is positively transverse to $\xi$.

Proof We perturb the surface such that it becomes generic and the elliptic singularities lie in the interior of $H(\xi)$ or in the interior of the foliated region. Furthermore, we assume in the following that there are no connections between different hyperbolic singularities of characteristic foliations.

If $D$ is a disc as in (b), then by the Poincare index theorem

$$
\begin{aligned}
\chi(D) & =e_{+}(D)+e_{-}(D)-h_{+}(D)-h_{-}(D) \\
e(\xi)(D) & =e_{+}(D)-e_{-}(D)-h_{+}(D)+h_{-}(D) .
\end{aligned}
$$

Subtracting these equalities we obtain $\chi(D)-e(\xi)[D]=2\left(e_{-}-h_{-}\right)$. In order to prove (b) it suffices to replace $D$ by an embedded disc $\widehat{D}$ with $e(\xi)[D]=e(\xi)[\hat{D}]$ and $\partial \hat{D}=\partial D$ such that $\hat{D}$ contains no negative elliptic singularities. Because $\xi$ is tight and $D$ is simply connected each cycle of $D(\xi)$ is the boundary of an integral disc.

In order to apply Lemma 3.7 or Lemma 3.8, we have to show that these integral discs do not intersect $\partial D$. So let $D^{\prime}$ be an integral discs of $\xi$ whose boundary lies on $D$ and assume that $D^{\prime}$ intersects $\partial D$. Then $D^{\prime} \cap D$ contains a union of leaves of $D(\xi)$ which form a smooth arc $\sigma$ with endpoints on $D$. Because $D(\xi)$ points out of $D$ at both ends of $\sigma$, this arc contains a singularity of $D(\xi)$. Note that no singularity of $D(\xi)$ in $D^{\prime} \cap D$ can be elliptic since all elliptic singularities lie in the interior of $H(\xi)$ or in the interior of the complement of $H(\xi)$. Thus there is exactly one hyperbolic singularity on $\sigma$ and $D^{\prime}$ contains both unstable leaves. Because $D^{\prime}$ is an integral disc, $D^{\prime} \cap D$ contains also both stable leaves together with their $\alpha$-limit sets. Since $D^{\prime}$ 
and $D$ are compact, $D \cap D^{\prime}$ contains a positive elliptic singularity. But this is absurd since $D^{\prime}$ would intersect the interior of the contact region.

We now choose particular cycles of $D(\xi)$ to which we apply Lemma 3.7 and Lemma 3.8: Define $\gamma \leq \gamma^{\prime}$ for two cycles $\gamma, \gamma^{\prime}$ of the characteristic foliation if $\gamma^{\prime}$ bounds an embedded disc containing $\gamma$. Let $\gamma_{m}$ be a cycle of $D(\xi)$ which is maximal with respect to $\leq$ and $D_{m}^{\prime}$ the integral disc of $\xi$ which bounds $\gamma_{m}$. In order to apply Lemma 3.7 and Lemma 3.8 we have to show that $D_{m}^{\prime}$ does not intersect $\partial D$. Note that the boundary of $D_{m}^{\prime}$ lies in the interior of $D$, so $D \cap D_{m}^{\prime}$ consists of integral curves of $D(\xi)$ which are cycles in the interior of or arcs whose endpoints lie on $\partial D$. This means in particular, that the holonomy of maximal cycles which are closed leaves of $D(\xi)$ is not trivial on the outer side of the cycle. The integral disc

Hence we obtain a disc $\widehat{D}$ whose characteristic foliation does not have closed cycles and all elliptic singularities are contained in $H(\xi)$. In particular, there are no integral discs of $\xi$ which pass through elliptic singularities of the characteristic foliation of $D$. Moreover, $e(\xi)[D]=e(\xi)[\hat{D}]$. From now on we will write $D$ instead of $\widehat{D}$.

Adapting arguments from [9] we eliminate one negative elliptic singularity $y$ : Let $U$ be a disc such that $\partial U$ is transverse to $D(\xi)$ and $y \in U$. According to Lemma 3.4 there is a Legendrian polygon $(Q, V, \alpha)$ covering $\overline{B(U)}$. In the present situation $V=\varnothing$ since $D(\xi)$ has no cycles or quasi-minimal sets.

Note that $B(U) \subset D$ because the characteristic foliation is pointing outwards along $\partial D$. After a small perturbation of $D$ we may assume that $\alpha$ identifies vertices of $\partial Q$ only if adjacent edges are also identified (for elliptic vertices see Figure 10). In this situation all boundary components of $\partial \overline{B(y)}$ are embedded piecewise smooth circles.

By construction $D(\xi)$ contains no cycles. Then every boundary component $\gamma_{o}$ of $\overline{B(y)}$ contains an elliptic singularity (which has to be positive). If all singularities of $D(\xi)$ on $\gamma_{o}$ are positive, then we obtain a contradiction to the tightness of $\xi$. Hence $\gamma_{o}$ contains a negative singularity which has to be hyperbolic. According to our assumptions it is a pseudovertex of the Legendrian polygon, ie its unstable leaf ends at $y$ while the other unstable leaf never meets $B(y)$.

Therefore the application of the elimination lemma (Lemma 3.5) does not create new cycles. We continue with the elimination of negative elliptic singularities until $e_{-}=0$. This finishes the proof of (b).

Now we prove of (a). First, we use Lemma 3.7 and Lemma 3.8 in order to decompose $S$ into a disjoint union of embedded spheres such that there are no cycles which contain hyperbolic singularities. In the following we consider each sphere individually, so we continue to write $S$. If $S(\xi)$ contains a closed leaf, then the claim follows immediately 
from the definition of tightness: Let $D_{1}, D_{2} \subset S$ be the two discs with $\partial D_{1}=\gamma=\partial D_{2}$. Then there is an integral disc $D^{\prime}$ of $\xi$ such that $\partial D^{\prime}=\gamma$. We orient $D^{\prime}$ such that $D_{1} \cup D^{\prime}$ is a cycle and denote by $-D^{\prime}$ the disc with the opposite orientation. Then $[S]=\left[D_{1} \cup D^{\prime}\right]+\left[\left(-D^{\prime}\right) \cup D_{2}\right]$ and the claim follows from (iii) of Definition 1.3 applied to $D_{1}, D_{2}$ :

$$
e(\xi)[S]=e(\xi)\left[D_{1} \cup D^{\prime}\right]+e(\xi)\left[\left(-D^{\prime}\right) \cup D_{2}\right]=0 .
$$

Finally, if $S(\xi)$ has neither closed leaves or cycles, then one can prove (a) using (b) when one considers complements of small discs around positive or negative elliptic singularities.

Consider a Legendrian polygon $(Q, V, \alpha)$ in $F \subset M$ when $\xi$ is a contact structure on $M$. Generically, the characteristic foliation on $F$ is of Morse-Smale type (cf [16]). In particular, there are no quasi-minimal sets. If the set of virtual vertices of the Legendrian polygon $(Q, V, \alpha)$ associated to $U$ is not empty, then by Lemma 3.6 one can create a canceling pair of singularities along on $\gamma_{v}$ for $v \in V$ such that all leaves which accumulated on $\gamma_{v}$ now accumulate on an elliptic singularity.

For this reason the case $V \neq \varnothing$ plays essentially no role when $\xi$ is a contact structure. If the $\omega$-limit set of $\gamma$ is contained in the fully foliated part of a confoliation, then it not possible to apply Lemma 3.6 (cf Section 5) in general. It is at this point where the proof of the Thurston-Bennequin inequalities for tight contact structures fails when one tries to adapt the arguments from [9] to tight confoliations and surfaces which are not simply connected.

We finish this section with a remark that will be useful later.

Remark 4.5 Let $\xi$ be a tight confoliation. For an embedded surface $F \subset M$ we define $d_{ \pm}(F)=e_{ \pm}(F)-h_{ \pm}(F)$. Note that if $F$ is a sphere, then $d_{+}(F)=d_{-}(F)=1$ by Theorem 4.4 and $\chi(F)=2$.

Part (b) Theorem 4.4 can be strengthened: Consider $\alpha$-limit set of stable leaves of positive hyperbolic singularities of $D^{\prime}$. Since $D^{\prime}(\xi)$ contains no cycles the $\alpha$-limit set is generically a positive elliptic singularity. Thus we may eliminate all negative elliptic and all positive hyperbolic singularities from $D^{\prime}(\xi)$. This implies

$$
\begin{aligned}
& d_{-}(D)=e_{-}(D)-h_{-}(D)=e_{-}\left(D^{\prime}\right)-h_{-}\left(D^{\prime}\right) \leq 0 \\
& d_{+}(D)=e_{+}(D)-h_{+}(D)=e_{+}\left(D^{\prime}\right)-h_{+}\left(D^{\prime}\right) \geq 0 .
\end{aligned}
$$

In a later application we shall consider discs such that $\partial D$ is negatively transverse to $\xi$. Then the two inequalities above will be interchanged. 


\subsection{Perturbations of tight confoliations on balls}

The proof Theorem 4.1 is given in the following sections. It has two main ingredients: First, we generalize taming functions on spheres in contact manifolds to confoliations. We show that the characteristic foliation on an embedded sphere $S$ can be tamed if $\xi$ is tight and that this remains true for contact structures $\xi^{\prime}$ which are close enough to $\xi$. Then we apply arguments from [17] to conclude that $\xi^{\prime} \mid B$ is tight if $\xi^{\prime}$ is a contact structure.

In the following sections $\xi$ will always be an oriented tight confoliation on $M$ and $S$ denotes an embedded oriented sphere. We do not consider foliations by spheres.

\subsubsection{Properties of $S(\xi)$ for tight confoliations $\xi$ In this section we prove a result} which will play an important role in the proof of Theorem 4.1. The following proposition is a generalization of Lemma 4.2.1 in [9].

Proposition 4.6 Let $S \subset M$ be an embedded sphere, $\xi$ a tight confoliation and $U \subset S$ a connected submanifold of dimension 2 whose boundary is transverse to $S(\xi)$ and $S(\xi)$ points out of $U$ everywhere. Let $(Q, V, \alpha)$ be the polygon covering the basin of $U$ and assume that $\partial U$ and $\partial Q$ have the same number of connected components.

For each connected component $\Gamma$ of $\partial Q$ one of the following is true.

(i) $\alpha(\Gamma)$ is an elliptic singularity and $\alpha(Q)$ is a neighbourhood of this singularity or $\alpha(\Gamma)$ is a cycle of $S(\xi)$ and $\alpha(Q)$ is a one-sided neighbourhood of this cycle.

(ii) $\Gamma$ contains a pseudovertex $w$ such that $\alpha(w)$ is a positive hyperbolic singularity and one stable leaf of $w$ is not contained in $\alpha(Q)$.

(iii) $(Q, \alpha, V)$ identifies pseudovertices $w_{1}, \ldots, w_{k}$. If $d_{+}(U)=1$, then $\alpha\left(w_{i}\right)$ is negative for all $i=1, \ldots, k$.

Proof Assume that $x \in F$ is a singularity of birth death type. We choose a neighbourhood $V$ of $x$ which has piecewise smooth boundary consisting of two segments which are both transverse to the characteristic foliation. The characteristic foliation points inwards along one smooth segment and outwards along the other smooth segment. After a small perturbation of $F$ in $V$ we may assume that there are only nondegenerate singularities. By the Poincaré index theorem, the number of hyperbolic singularities equals the number of elliptic singularities (in $V$ ). Moreover, all these singularities have the same sign as $x$. Let us assume that $x$ is negative.

At most one unstable leaf of a hyperbolic singularity can escape from $V$ since otherwise there must be a positive elliptic singularity. Using the elimination lemma and the 
Lemmas from Section 3.4 we can successively eliminate all hyperbolic singularities from $V$ and the resulting surface is $C^{0}$-close to $F$ (because a neighbourhood of $x$ in $M$ is tight by Proposition 2.5, so all surgery discs are contained in the neighbourhood of $x$ ). Now $V$ contains no singularities at all. If one chooses $V$ to be disjoint from all stable and unstable leaves of hyperbolic singularities of $F(\xi)$, then the basin $B(U)$ is unchanged after this operation, but the birth-death type singularity disappeared.

It was shown in Lemma 3.4 that $\overline{B(U)}$ is covered by a Legendrian polygon $(Q, V, \alpha)$. Recall that $\alpha$ is defined only on $\Gamma \backslash(\Gamma \cap V)$, but we shall denote $\alpha(\Gamma \backslash(\Gamma \cap V))$ by $\alpha(\Gamma)$.

First, we reduce the situation to the case when $V=\varnothing$. By the theorem of PoincaréBendixon, the $\omega$-limit sets $\gamma_{v}, v \in V$ are cycles. Because $\xi$ is tight, these cycles bound integral discs $D_{v}, v \in V$ of $\xi$ and we can apply Lemma 3.7 or Lemma 3.8. Since $D_{v}$ may intersect $U$ it is also necessary to consider cycles in $U$.

Let $\gamma_{i}$ be a cycle of $S(\xi)$ which is contained in $D_{v}$. We assume that the disc $D_{i} \subset D_{v}$ bounded by $\gamma_{i}$ intersects $S$ only along $\gamma_{i}$. The cycle $\gamma_{i}$ is either contained in $U$ or in the complement of $U$.

We begin with the case $\gamma_{i} \subset U$. We obtain two embedded spheres $S^{\prime}, S^{\prime \prime}$ by cutting along $\gamma_{i}$. When we use Lemma 3.7, the subset $U \subset S$ induces two subsets $U^{\prime} \subset S^{\prime}$, $U^{\prime \prime} \subset S^{\prime \prime}$ such that $U^{\prime}$ respectively $U^{\prime \prime}$ contains one positive respectively one negative singularity in addition to singularities which were already present in $S, \partial U^{\prime}$ respectively $\partial U^{\prime \prime}$ is transverse to $S^{\prime}(\xi)$ respectively $S^{\prime \prime}(\xi)$ and the characteristic foliation points outwards. The pseudovertices of the Legendrian polygons associated to the basins of $U^{\prime}, U^{\prime \prime}$ coincide with the pseudovertices of $(Q, \alpha, V)$. If $d_{+}(U)=1$, then

$$
\begin{gathered}
d_{+}\left(U^{\prime}\right)+d_{+}\left(U^{\prime \prime}\right)=d_{+}(U)+1 \\
d_{+}\left(S^{\prime} \backslash U^{\prime}\right)+d_{+}\left(U^{\prime}\right)=d_{+}\left(S^{\prime}\right)=1 \\
d_{+}\left(S^{\prime \prime} \backslash U^{\prime \prime}\right)+d_{+}\left(U^{\prime \prime}\right)=d_{+}\left(S^{\prime \prime}\right)=1 .
\end{gathered}
$$

Notice that $\left(S^{\prime} \backslash U^{\prime}\right) \cup\left(S^{\prime \prime} \backslash U^{\prime \prime}\right)=S \backslash U$ and $\partial(S \backslash U)$ is negatively transverse to $S_{\xi}$. It follows from Remark 4.5 that $d_{+}\left(S^{\prime} \backslash U^{\prime}\right) \leq 0$ and $d_{+}\left(S^{\prime \prime} \backslash U^{\prime \prime}\right) \leq 0$. Together with (5) this implies $d_{+}\left(U^{\prime}\right)=d_{+}\left(U^{\prime \prime}\right)=1$.

If we applied Lemma 3.8 and the hyperbolic singularity was positive, respectively negative, then $h_{+}\left(U^{\prime} \cup U^{\prime \prime}\right)=h_{+}(U)-1$, respectively $e_{+}\left(U^{\prime} \cup U^{\prime \prime}\right)=e_{+}(U)+1$. Hence $d_{+}\left(U^{\prime}\right)+d_{+}\left(U^{\prime \prime}\right)=d_{+}(U)+1$ and $d_{+}(U)=1$ implies $d_{+}\left(U^{\prime \prime}\right)=1$ as above.

When $\gamma_{i}$ lies in the complement of $U$, cutting along $\gamma_{i}$ will not affect $U$ or $d_{+}(U)$ but the basin of $U$ can change: We might replace a virtual vertex by a vertex which 
corresponds to an elliptic singularity, or after the surgery process some boundary components of the Legendrian polygon might be mapped to a negative elliptic singularity while they accumulated on a cycle before. The pseudovertices are not affected. Note also that if $\alpha(Q)$ is a one-sided neighbourhood of a cycle $\gamma_{v}$, then the Legendrian polygon which results from the surgery along $\gamma_{v}$ will be a neighbourhood of the negative elliptic singularity which results from surgery process.

After finitely many steps we obtain a finite union of embedded spheres $S_{j}$ and subsets $U_{j}$ with the same properties as $U \subset S$ or $U_{j}=S_{j}$. The associated Legendrian polygons $\left(Q_{j}, V_{j}, \alpha_{j}\right)$ have no virtual vertices, ie $V_{j}=\varnothing$. Therefore it suffices to prove the claim when $V=\varnothing$. Let $\Gamma$ be a boundary component of $Q$. If $\Gamma$ contains no pseudovertex, then we have the following:

(1) Either $\alpha(\Gamma)$ is an elliptic singularity $x$ of $S(\xi)$ and $\alpha(Q)$ is a neighbourhood of $x$, or $\alpha(\Gamma)$ is a cycle and $\alpha(Q)$ is a one-sided neighbourhood of the cycle. These cases correspond to (i).

(2) There is a pseudovertex $w$ on $\Gamma$ such that a stable leaf $\eta_{w}$ of $\alpha(w)$ is not contained in $\alpha(Q)$. In this case we will obtain cycles as in case (ii).

(3) Both stable leaves of all pseudovertices on $\Gamma$ are contained in $\alpha(Q)$. These cycles will turn out to have the property described in (iii).

Consider case (2). Let $W$ be the connected component of $\overline{S \backslash \alpha(Q)}$ containing $\eta_{w}$. The boundary of $W$ is a finite union of Legendrian segments of $S(\xi)$. Since two Legendrian segments of $\partial W$ point away from $\alpha(w) \in \partial W$ there must be a point $y \in \partial W$ which is the $\omega$-limit set of two Legendrian segments of $\partial W$.

Assume that we can choose $y$ to be elliptic. Then $y$ is negative and we may assume that $y$ lies in the interior of the contact region of $\xi, \partial W$ contains no corners and this perturbation does note affect the image of the pseudovertices on $\partial W$. If all hyperbolic singularities on $\partial W$ which correspond to pseudovertices were negative, then $\partial W$ would bound a disc in $S$ and (after eliminating pairs of negative singularities using Lemma 3.5 removing corners from $\partial W$ by perturbations of $S$ ) one would obtain a disc $D$ satisfying all requirements from Definition 1.3 but for which there is no integral disc $D^{\prime}$. Thus the tightness of $\xi$ ensures the existence of a positive pseudovertex $w^{\prime}$ on $\Gamma$ such that one stable leaf of $\alpha\left(w^{\prime}\right)$ is not contained in $\alpha(Q)$.

Now assume that $y$ has to be hyperbolic. In this case, one unstable leaf is contained in $W$ while the other unstable leaf is contained in $\alpha(Q)$. By Lemma 3.7 and Lemma 3.8 we may assume that the $\omega$-limit set of the latter unstable leaf is an elliptic singularity, again this singularity of $S(\xi)$ has to be negative. By a small perturbation of $S$ near $y$ we can arrange that the part of $\partial W$ which ended at $y$ now ends at the elliptic 
singularity. Thus we have reduced the present situation to the situation of the previous paragraph without introducing new pseudovertices. Hence we conclude that there is a pseudovertex $w^{\prime}$ on $\Gamma$ such that one stable leaf of $w^{\prime}$ is not contained in $\alpha(Q)$ and $\alpha\left(w^{\prime}\right)$ is positive.

It remains to treat case (3). Let $w_{1} \in \Gamma$ be a pseudovertex on $\Gamma$. Both stable leaves of $\alpha\left(w_{1}\right)$ are contained in $\alpha(Q)$, thus there is another vertex $w_{2} \in \Gamma$ such that $\alpha\left(w_{1}\right)=\alpha\left(w_{2}\right)$. If $w_{2}$ is a pseudovertex, then $\alpha$ identifies pseudovertices. Otherwise $w_{2}$ is a corner and we denote it by $w_{2}^{\prime}$. Let $W$ be the connected component of $\overline{S \backslash \alpha(Q)}$ which contains $\alpha\left(w_{1}\right)$ in its closure. As before, the boundary of $W$ is a finite union of segments of $S(\xi)$. In particular, it is piecewise smooth. By assumption $W$ does not contain any stable leaf of a hyperbolic singularity of $S(\xi)$ in its interior. Since all elliptic singularities on $\partial W \subset \alpha(Q)$ have to be negative, all points on $\partial W$ from which two segments of $\partial W$ point away have to be hyperbolic and one stable leaf of such a hyperbolic singularity would be contained in $W$ and not in $\alpha(Q)$. This contradicts our hypothesis on $\Gamma$. Hence $(Q, \alpha, V)$ is not injective in this case.

Assume $d_{+}(U)=1$ and let $w_{1}, \ldots, w_{k}, k \geq 2$ be the pseudovertices on $\Gamma$ such that $\alpha\left(w_{i}\right)$ lies on $\partial W$ for $i=1, \ldots, k$.

We first treat the case $k=2$ and $\alpha\left(w_{1}\right)=\alpha\left(w_{2}\right)$. After a small perturbation of $S$ in the complement of $U$ we may assume that the $\alpha$-limit sets of the stable leaves $\eta, \eta^{\prime}$ of $\alpha\left(w_{1}\right)$ are contained in $U$.

We may assume that neither $\mathcal{A}(\eta)$ or $\mathcal{A}\left(\eta^{\prime}\right)$ is a hyperbolic singularity or a singularity of birth-death type. By the Poincaré-Bendixon theorem $\mathcal{A}(\eta)$ is either an elliptic singularity or a cycle. The same is true for $\mathcal{A}\left(\eta^{\prime}\right)$. Using Lemma 3.7 and Lemma 3.8 we can ensure that $\mathcal{A}(\eta)$ is an elliptic singularity, which has to be positive (again this does not affect the hypotheses on $d_{+}$). Note that $\eta, \eta^{\prime}$ lie in the same connected component of the two spheres obtained by the surgery along cycles in $U$.

For the same reason we may assume that the $\alpha$-limit set of each stable leaf of hyperbolic singularities in $U$ is an elliptic singularity in $U$. Under these conditions the hypotheses $d_{+}(U)=1$ implies that the graph formed by positive singularities (except birth-death type singularities) and stable leaves of hyperbolic singularities is a connected tree $T_{+}$. In particular, the stable leaves of $\alpha\left(w_{1}\right)$ together with edges of $T_{+}$forms a closed Legendrian curve $\gamma$. If $\alpha\left(w_{1}\right)$ is positive, $\gamma$ bounds a disc and all singularities on $\gamma$ are positive and $\gamma$ contains an elliptic singularity. Then we can arrange that this elliptic singularity lies in the contact region to obtain a contradiction to the tightness of $\xi$. Thus $\alpha\left(w_{1}\right)$ is negative.

A very similar argument applies when $w_{1}, \ldots, w_{k}$ lie on a nontrivial cycle $\gamma_{w_{1}} \ldots w_{k}$. Let $\eta$ be the stable leaf of $w_{i}$ which is contained in the interior of $\alpha(Q)$ and let $\eta^{\prime}$ 
be the other stable leaf. Without loss of generality we assume $i>1$. Now $\eta^{\prime}$ can be connected by Legendrian segments in $\partial W$ to $\alpha\left(w_{i-1}\right)$ such that no pseudovertices are mapped to points lying between $\alpha\left(w_{i}\right)$ and $\alpha\left(w_{i-1}\right)$. We extend $\eta^{\prime}$ by this piece of $\partial W$ and the stable leaf of $\alpha\left(w_{i-1}\right)$ which is contained in $\alpha(Q)$. As above, we obtain a simple closed Legendrian curve passing through $\alpha\left(w_{i}\right)$ and $\alpha\left(w_{i-1}\right)$. By construction, one Legendrian segment of $\gamma$ is a stable leaf of $\alpha\left(w_{i-1}\right)$ while the other Legendrian segment of $\gamma$ which ends on $\alpha\left(w_{i-1}\right)$ is an unstable leaf. Thus we can perturb $S$ outside of a neighbourhood of $\alpha\left(w_{i}\right)$ such that we obtain a cycle $\gamma^{\prime}$ which contains only positive hyperbolic singularities unless $\alpha\left(w_{i}\right)$ is negative. As above, the tightness of $\xi$ implies that $\alpha\left(w_{i}\right)$ is negative.

Since every connected component of $Q$ belongs to one of the types (1),(2) or (3) this proves the claim.

Note that we did not really use the assumption that $\partial Q$ and $\partial U$ have the same number of connected components. However, the main use of the lemma will be the construction of a taming function on $S$. In that construction additional (ie those arising from adding 1-handles in the proof of Lemma 3.4) components of $\partial Q$ have to be treated in a slightly different fashion.

4.2.2 Taming functions for characteristic foliations on spheres Taming functions for characteristic foliations were introduced by Eliashberg in [9]. In this section we extend the definition of taming functions so that it can be applied to spheres embedded in manifolds carrying a tight confoliation.

Let $S$ be an embedded sphere in a confoliated manifold such that the singularities of the characteristic foliation $S(\xi)$ are nondegenerate or of birth-death type. This assumption holds in particular for spheres in a generic 1-parameter family of embeddings. In order to define taming functions we still need to introduce more terminology.

Definition 4.7 A cycle $\gamma$ of $S(\xi)$ is an internal subcycle if there is another cycle $\gamma^{\prime}$ of $S(\xi)$ such that $\gamma \cap \gamma^{\prime}$ is not empty and the integral disc which bounds $\gamma^{\prime}$ contains the integral disc which bounds $\gamma$. A leaf $\gamma$ of $S(\xi)$ is called internal if it contained in an internal cycle. We say that a hyperbolic singularity on $\gamma$ is essential if it is not lying on an internal subcycle of $\gamma$.

The union of singular points and cycles of $S(\xi)$ will be denoted by $\Sigma(S)$.

The set $\Sigma(S)$ is compact. An example of an internal subcycle is shown in Figure 12. Note that one can create internal cycles intersecting a fixed cycle of $S(\xi)$ with arbitrary sign using an inverse of the construction explained in Lemma 3.8. 
Let $U \subset S$ be a compact submanifold of dimension 2 in $S$ whose boundary is piecewise smooth and does not intersect $\Sigma(S)$. Moreover, we assume that every connected component $\Gamma \subset \partial U$ satisfies one of the following conditions:

(1) $\Gamma$ is smooth and either transverse or tangent to $S(\xi)$.

(2) $\Gamma$ is piecewise smooth, each smooth segment is transverse to $S(\xi)$ and intersects one separatrix of a hyperbolic singularity in $U$.

(3) $U$ is a disc and a neighbourhood of a birth-death type singularity of $S(\xi)$ such that $\partial U$ consists of two smooth segments transverse to $S(\xi)$.

Definition 4.8 A function $f: U \rightarrow \mathbb{R}$ is a taming function for $S(\xi)$ if it has the following properties.

(o) If a component $\Gamma \subset \partial U$ belongs to the class (1), then $f$ is constant along $\Gamma$. If $\Gamma$ is of class (2) or (3) we require that $\left.f\right|_{\Gamma}$ has exactly one critical point in the interior of each of the smooth segments of $\Gamma$.

(i) The union of the singular points of $S(\xi)$ with all points on internal leaves coincides with the set of critical points of $f$. The function is strictly increasing along leaves of $S(\xi)$ which are not part of a cycle and $f$ is constant along cycles of $S(\xi)$.

(ii) Positive respectively negative elliptic points of $S(\xi)$ are local minima respectively maxima of $f$.

(iii) If the level set $\{f=C\}$ contains only hyperbolic singularities, then as $a$ increases from $C-\varepsilon$ to $C+\varepsilon$ for sufficiently small $\varepsilon>0$ the number of closed connected components of $\{f=C\}$ changes by $h_{-}(\{f=C\})-h_{+}(\{f=C\})$.

Requirement (i) in Definition 4.8 is slightly more complicated than one might expect. Figure 12 gives an example of a sphere $S$ in $\mathbb{R}^{3}$ equipped with the foliation by horizontal planes and cooriented by $d z$ where $z$ is the vertical coordinate: The left part of the figure shows the intersection of $S$ with a vertical plane while the right part depicts a part of $S(\xi)$. The internal subcycle is the interior part of the thickened curve. If one requires that only singular points of $S(\xi)$ are critical points of the taming function, then $S(\xi)$ cannot be tamed although the confoliation in question is tight.

Assume that $(X, \omega)$ is a symplectic filling of $(M, \xi)$ and a compatible almost complex structure on $M$ is fixed such that $\xi$ consists of complex lines. By Theorem 1 of Hind [20] an embedded 2-sphere $S \subset M$ can be filled by holomorphic discs when the embedding of $S$ satisfies several technical conditions. The singular foliation in the formulation of Theorem 1 in [20] is very similar to the singular foliation formed by level sets of a taming function. The appearance of internal cycles should be compared with Remark 2 in [20]. 

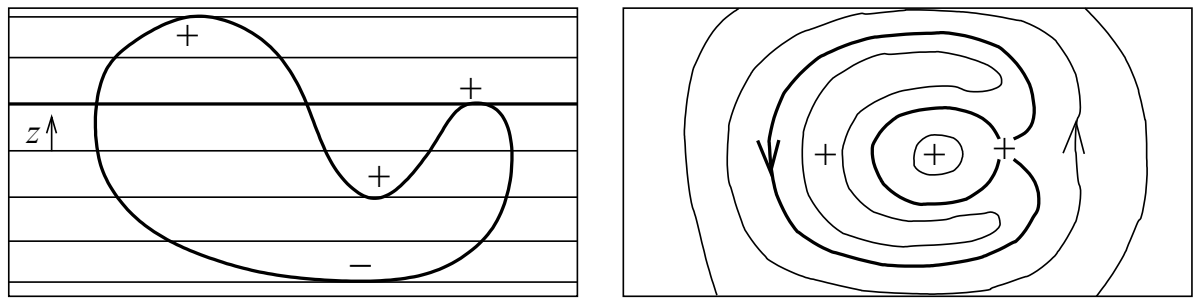

Figure 12

\subsubsection{Construction and perturbations of taming functions Let $S \subset M$ an em-} bedded oriented 2-sphere. The tightness of $\xi$ leads to several restrictions on the combinatorics of the cycles of $S(\xi)$ and their holonomy. This will be used to construct a taming function for $S(\xi)$.

Recall that the orientations of $S$ and $\xi$ induce an orientation of $S(\xi)$ and integral surfaces of $\xi$ are oriented by the orientation of $\xi$. If $\gamma$ is a cycle of $S(\xi)$, then by tightness there is an integral disc $D_{\gamma}$ of $\xi$ such that $\partial D_{\gamma}=\gamma$ but the orientation of $\partial D_{\gamma}$ as boundary of $D_{\gamma}$ does not coincide with the orientation of $\gamma$ in general. Recall also that $D_{\gamma}$ is uniquely determined because $\xi$ is not a foliation by spheres.

For a 2-dimensional submanifold $U \subset S$ with piecewise smooth boundary we define the following quantities:

$$
\begin{aligned}
d_{+}(U)= & e_{+}(U)-h_{+}(U) \\
N_{-}(U)= & \text { number of connected components } \Gamma \text { of } \partial U \text { where } S(\xi) \text { points } \\
& \text { transversally into } U \text { or } \Gamma \text { is tangent to } S(\xi) \text { and } \Gamma \text { is potentially } \\
& \text { repulsive on the side of } U \\
N_{S}(U)= & \text { number of boundary components of } \partial U \text { through which stable } \\
& \text { leaves of negative hyperbolic singularities enter } \\
P_{S}(U)= & \text { number of stable leaves of positive hyperbolic singularities in } U \\
& \text { which intersect } \partial U
\end{aligned}
$$

Lemma 4.9 For each path connected component $\Sigma_{0}$ of $\Sigma(S)$ there is a neighbourhood $U_{0}$ of $\Sigma_{0}$ and a taming function $f: U_{0} \rightarrow \mathbb{R}$ such that no connected component of $\partial U_{0}$ is tangent to $S(\xi)$ and

$$
d_{+}\left(U_{0}\right)=1-N_{-}\left(U_{0}\right)-P_{S}\left(U_{0}\right)-N_{s}\left(U_{0}\right) .
$$

Proof We will construct $U_{0}$ and $f: U_{0} \rightarrow \mathbb{R}$ inductively. The starting point are connected cycles $\gamma$ and singularities of $S(\xi)$ in $\Sigma_{0}$ which belong to the following classes. 
(i) Positive elliptic singularities and hyperbolic or birth-death type singularities which do not belong to a cycle.

(ii) Closed leaves with sometimes attractive (nontrivial) one-sided holonomy.

(iii) Cycles containing hyperbolic singularities which satisfy the following conditions: - The only cycle of $S(\xi)$ containing $\gamma$ is $\gamma$.

- If $\gamma_{0} \subset \gamma$ is a subcycle with potentially attractive one-sided holonomy, then this one-sided holonomy is not trivial.

If the positive elliptic singularity $y$ in (i) is dynamically hyperbolic, then it is a source and there is a taming function on a neighbourhood $U$ whose boundary is transverse to $S(\xi)$. If the elliptic singularity is not dynamically hyperbolic, then one obtains a taming function using the holonomy of an interval $[0, \eta), \eta>0$ which is transverse to $S(\xi)$ except at $y$ and $y$ corresponds 0 (cf Lemma 3.1). If the holonomy is nontrivial, then we can choose the domain $U$ of the taming function such that $\partial U$ is transverse to $S(\xi)$ and $U_{0}=U$. Otherwise we choose $U$ such that $\partial U$ is a closed leaf of $S(\xi)$. The domain $U$ satisfies (6).

If $x$ is a hyperbolic singularity or a singularity of birth-death type, then the existence of a taming function on a neighbourhood $U_{0}$ which satisfies (6) is obvious.

For a closed leaf $\gamma$ of $S(\xi)$ as in (ii) we choose an embedded interval $(-\eta, \eta), \eta>0$ transverse to $S(\xi)$ such that 0 corresponds to a point in $\gamma$ and $(-\eta, 0]$ corresponds to the side where the holonomy of $\gamma$ is sometimes attractive. This choice determines $f$ along the transverse segment and $f$ can be extended to a taming function on a neighbourhood of $\gamma$. If the holonomy on the side $\{f \geq 0\}$ is nontrivial (respectively trivial) we choose $U$ to be an annulus with transverse boundary (respectively such that $\partial U \cap\{f>0\}$ is a leaf of $S(\xi)$ and the other component of $\partial U_{0}$ is transverse to $S(\xi)$ ). Thus $N_{-}(U)=1$ and $U$ contains no singular points of $S(\xi)$. This means that (6) holds for $U$. If $\partial U$ is transverse to $S(\xi)$ we set $U_{0}=U$.

Now let $\gamma$ be a cycle as in (iii). For each subcycle with potentially attractive (respectively repelling) one-sided holonomy fix a transversal $(-\varepsilon, 0]$ (respectively $[0, \varepsilon)$ ) with 0 lying on $\gamma$ and construct taming functions on collars of discs bounding the subcycle.

Assume that $\gamma$ contains a corner such that only one stable leaf of the hyperbolic singularity is part of a cycle. Then the level sets of $f$ near $\gamma$ can be chosen as suggested in Figure 13. The thick curve represents a critical level of $f$ while the dashed curve corresponds to a regular level of $f$. In Figure 13 the one-sided holonomy along the cycle is repulsive.

Whenever the germ of the one-sided holonomy is nontrivial, we choose the boundary corresponding boundary component of the domain $U$ of $f$ to be transverse to $S(\xi)$, 

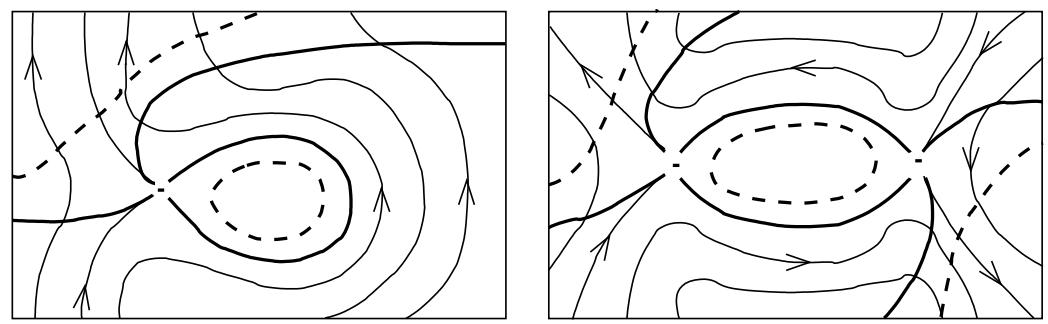

Figure 13

otherwise we choose the boundary of the domain to be tangent to a leaf of $S(\xi)$. In this way, we obtain a function $f: U \rightarrow \mathbb{R}$ on a neighbourhood of $\gamma$.

Before we continue with the construction of $U_{0}$, we show that $f: U \rightarrow \mathbb{R}$ satisfies the requirements (i) and (iii) from Definition 4.8 and (6).

By construction, $f$ is constant along cycles and increasing along leaves of $S(\xi)$ which are not part of cycles. Singular points of $S(\xi)$ clearly are critical points of $f$ and all critical points of $f$ lie on cycles of $S(\xi)$. In order to show that requirement (i) of Definition 4.8 is satisfied by $f$ we first consider an internal leaf $\gamma_{0} \subset \gamma$.

Let $D_{0,1}, D_{0,2} \subset S$ be discs with disjoint interiors such that $\gamma_{0} \subset \partial D_{0, i}$ for $i=1,2$ and whose interiors contain no subcycle of $\gamma$. By tightness $\partial D_{0,1}, \partial D_{0,2}$ bound integral discs $D_{0,1}^{\prime}, D_{0,2}^{\prime}$ of $\xi$ whose intersection with $S$ consists of cycles which also lie in the interior of $D_{0,1}$ and $D_{0,2}$. By our assumptions this intersection is empty, therefore the one-sided holonomy along $\partial D_{0,1}, \partial D_{0,2}$ is well defined.

Now one of the cycles, say $\partial D_{0,1}$, is oriented as the boundary of $D_{0,1}^{\prime}$ while the orientation of the other cycle, say $\partial D_{0,2}$, is the opposite of the orientation of the integral disc bounding it. Moreover, the collars of $\partial D_{0,1}$ and $\partial D_{0,2}$ lie on opposite sides of $D_{0,1}^{\prime} \cup D_{0,2}^{\prime}$. It follows from Lemma 2.1 that the one-sided holonomy along $\partial D_{0,1}$ is potentially attractive (repulsive) if and only if the same is true for $D_{0,2}$. Hence all points of $\gamma_{0}$ are critical points of $f$.

Conversely, if $\gamma_{0}$ is not an internal leaf, then there are cycles $\gamma_{0,1}, \gamma_{0,2}$ such that the one-sided holonomy along these cycles is well defined on opposite sides of $\gamma_{0}$. Again there are integral discs $D_{0,1}^{\prime}, D_{0,2}^{\prime}$ of $\xi$ which bound $\gamma_{0,1}, \gamma_{0,2}$, but now one of these discs is contained in the other one. In contrast to the previous case, both $\gamma_{0,1}, \gamma_{0,2}$ are oriented as the boundary of the corresponding integral disc or their orientations are opposite to that orientation. Therefore Lemma 2.1 implies that the holonomy along $\gamma_{0,1}$ is potentially attractive if and only if $\gamma_{0,2}$ is potentially repelling and vice versa. This implies (i) from Definition 4.8 for $f: U \rightarrow \mathbb{R}$. 
Using induction on the number of hyperbolic singularities in $\gamma$ we now prove (iii) from Definition 4.8 and (6) for $f: U \rightarrow \mathbb{R}$. We have already treated the case when $\gamma$ contains no hyperbolic singularity.

Given a cycle $\gamma$ and a fixed hyperbolic singularity $x_{0}$ we isotope $S$ in a neighbourhood of $x_{0}$, the resulting sphere will be denoted by $S^{\prime}$. We want to achieve that segments of $S(\xi)$ in $S \cap S^{\prime}$ which ended at $x_{0}$ before the perturbation are now connected be nonsingular segments of $S^{\prime}(\xi)$. In this way obtain a cycle $\gamma^{\prime}$ or a pair of cycles $\gamma^{\prime}, \gamma^{\prime \prime}$ on $S^{\prime}$ containing one singularity less than $\gamma$.

In order to construct the desired isotopy one moves $x_{0}$ away from the integral surface of $\xi$ which contains the cycle $\gamma$. If $x_{0}$ is part of an internal cycle or not all stable/unstable leaves of $x_{0}$ are contained in $\gamma$, then we move $x_{0}$ into the interior of an integral surface of $\xi$ and then slightly above or below the integral surface with respect to the coorientation of $\xi$. Choosing to push upwards or downwards one can make sure that one obtains a cycle on the perturbed surface which is contained in the interior of the integral surface of $\xi$ which contains $\gamma$. Figure 14 shows one particular instance of the isotopy in a neighbourhood of $x_{0}$. In that figure, we move $x_{0}$ downwards. In the left part of the figure all lines are part of $S$ while in the right part the straight lines do not belong to $S^{\prime}$. The cycles $\gamma$ respectively $\gamma^{\prime}$ correspond to the thickened lines in the left respectively right part of Figure 14.
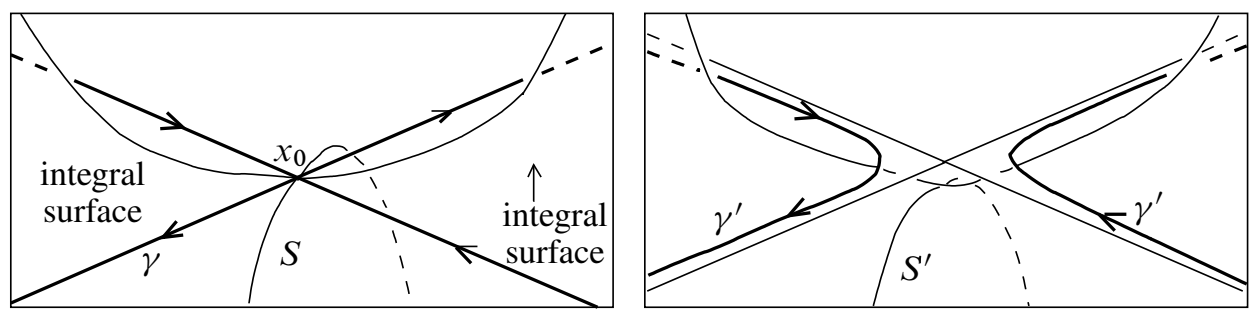

Figure 14

In the following we explain the relation between properties of $\gamma$ and the perturbed cycle.

If there is a hyperbolic singularity $x_{0} \in \gamma$ such that $\gamma$ contains only one stable leaf $\gamma_{0}$ of $x_{0}$ (as in Figure 13, for example), then $x_{0}$ is automatically an essential singularity on $\gamma$. Because $\xi$ is tight, there is an integral disc $D^{\prime}$ of $\xi$ whose boundary is contained in $\gamma$ and $\gamma_{0} \subset \partial D^{\prime}$. As before, $\gamma_{0}$ is contained in a cycle $\gamma_{0}^{c}$ with well defined one-sided holonomy (on the side of $\gamma$ which does not contain the stable leaf of $x$ ).

The following table contains the sign of $x_{0}$ depending on the nature of the holonomy along $\gamma_{0}^{c}$ and the orientation of $\gamma_{0}^{c}$ compared with the orientation of $\partial D^{\prime}$. We also 
indicate the side of $D^{\prime}$ (with respect to the coorientation given by the coorientation of $\xi$ ) on which the holonomy along $\gamma_{0}^{c}$ is well defined, this information follows immediately from Lemma 2.1. Using this, the sign of $x_{0}$ is easily determined by our orientation convention for $S(\xi)$.

\begin{tabular}{c|c|c} 
& $\gamma_{0}$ oriented as $\partial D^{\prime}$ & $\gamma_{0}$ oriented as $-\partial D^{\prime}$ \\
\hline$\gamma_{0}^{c}$ attractive & $\begin{array}{c}\text { domain of holonomy } \\
\text { above } D^{\prime}, x_{0} \text { is positive }\end{array}$ & $\begin{array}{c}\text { domain of holonomy } \\
\text { below } D^{\prime}, x_{0} \text { is positive }\end{array}$ \\
\hline$\gamma_{0}^{c}$ repulsive & $\begin{array}{c}\text { domain of holonomy } \\
\text { below } D^{\prime}, x_{0} \text { is negative }\end{array}$ & $\begin{array}{c}\text { domain of holonomy } \\
\text { above } D^{\prime}, x_{0} \text { is negative }\end{array}$
\end{tabular}

This table shows that when $\gamma_{0}^{c}$ has attractive holonomy, then $x_{0}$ is positive. It follows from he construction of $f$ that two components of the level set $f^{-1}(c)$ merge when $c$ passes $f(\gamma)$. This finishes the inductive step for this case.

Now assume that there are exactly two integral discs $D_{1}^{\prime}, D_{2}^{\prime}$ of $\xi$ with $x_{0} \in \partial D_{i}^{\prime}, i=1,2$ whose boundaries are cycles $\gamma_{1}^{\prime}, \gamma_{2}^{\prime}$ with well defined one-sided holonomy of $S(\xi)$. In this case, $x_{0}$ is essential. An example of this configuration is a cycle in the form of a figure-eight and the subcycles bound integral discs with disjoint interior.

Each stable leaf of $x_{0}$ is contained in exactly one of the discs $D_{1}^{\prime}, D_{2}^{\prime}$. In particular, the one-sided holonomy of $\partial D_{1}^{\prime}$ is potentially attractive if and only if the same is true for $\partial D_{2}^{\prime}$ and the domains of the one-sided holonomies lie on the same side of $D_{1}^{\prime} \cup D_{2}^{\prime}=: D^{\prime}$.

The following table summarizes the relationship between the sign of $x_{0}$, the one-sided holonomies and the orientation of $\gamma_{i}$ respectively $D_{i}^{\prime}$. It shows that two connected components of $f^{-1}(c)$ merge at $x_{0}$ when $c$ passes the critical value $f(\gamma)$ if and only if $x_{0}$ is positive. Otherwise the level set splits into two connected components at $x_{0}$.

\begin{tabular}{c|c|c} 
& $\gamma_{i}$ oriented as $\partial D_{i}^{\prime}$ & $\gamma_{i}$ oriented as $-\partial D_{i}^{\prime}$ \\
\hline$\gamma_{i}$ attractive & $\begin{array}{c}\text { domain of holonomy } \\
\text { above } D_{i}^{\prime}, x_{0} \text { is positive }\end{array}$ & $\begin{array}{c}\text { domain of holonomy } \\
\text { below } D_{i}^{\prime}, x_{0} \text { is positive }\end{array}$ \\
\hline$\gamma_{i}$ repulsive & $\begin{array}{c}\text { domain of holonomy } \\
\text { below } D_{i}^{\prime}, x_{0} \text { is negative }\end{array}$ & $\begin{array}{c}\text { domain of holonomy } \\
\text { above } D_{i}^{\prime}, x_{0} \text { is negative }\end{array}$
\end{tabular}

In order to show that $f: U \rightarrow \mathbb{R}$ satisfies (iii) of Definition 4.8 we now treat the case when one stable leaf of $x_{0}$ is part of a cycle $\gamma_{b}=\partial D_{b}^{\prime}$ with one-sided holonomy while the other stable leaf is also part of a cycle $\gamma_{i n}=\partial D_{i n}^{\prime}$ with well defined one-sided holonomy such that $D_{i n}^{\prime} \subset D_{b}^{\prime}$ and $D_{i n}^{\prime}$ contains exactly one stable leaf of $x_{0}$. An example of such a configuration is the thickened curve in the right part of Figure 12. 
Now the domains of the one-sided holonomy of $\gamma_{b}$ and $\gamma_{i n}^{\prime}$ lie on the same side of $D_{b}$ while $\gamma_{i n}=-\partial D_{i n}^{\prime}$ if and only if $\gamma_{b}=\partial D_{b}^{\prime}$ and the holonomy along $\gamma_{i n}$ is attractive if and only if the holonomy along $\gamma_{b}$ is repulsive.

\begin{tabular}{l|c}
$\gamma_{i n}$ attractive \\
$\Rightarrow \gamma_{b}$ repulsive & $\begin{array}{c}\gamma_{\text {in }} \text { oriented as } \partial D_{i n}^{\prime} \Rightarrow \gamma_{b} \text { oriented as }-\partial D_{b}^{\prime} \\
\text { domain of the holonomy of } \gamma_{i n} \text { above } D_{b}^{\prime}\end{array}$ \\
\hline $\begin{array}{c}\gamma_{i n} \text { repulsive } \\
\Rightarrow \gamma_{b} \text { attractive }\end{array}$ & $\begin{array}{c}\text { domain of the holonomy of } \gamma_{i} \text { below } D_{b}^{\prime} \\
\text { domain of the holonomy of } \gamma_{b} \text { below } D_{b}^{\prime} \\
x_{0} \text { negative }\end{array}$ \\
$\begin{array}{c}\gamma_{i n} \text { oriented as }-\partial D_{\text {in }}^{\prime} \Rightarrow \gamma_{b} \text { oriented as } \partial D_{b}^{\prime} \\
\Rightarrow \gamma_{b} \text { repulsive }\end{array}$ & $\begin{array}{c}\text { domain of the holonomy of } \gamma_{i n} \text { below } D_{b}^{\prime} \\
\text { domain of the holonomy of } \gamma_{b} \text { below } D_{b}^{\prime} \\
x_{0} \text { positive }\end{array}$ \\
\hline$\gamma_{i}$ repulsive \\
$\Rightarrow \gamma_{b}$ attractive & $\begin{array}{c}\text { domain of the holonomy of } \gamma_{i n} \text { above } D_{b}^{\prime} \\
\text { domain of the holonomy of } \gamma_{b} \text { above } D_{b}^{\prime} \\
x_{0} \text { negative }\end{array}$
\end{tabular}

The perturbation of $S$ can be arranged such that the stable respectively unstable leaf of $x_{0}$ lying in $\gamma_{\text {in }}$ gets connected to the unstable respectively stable leaf of $x_{0}$ which is part of $\gamma_{b}$. The resulting cycle $\gamma^{\prime} \subset S^{\prime}$ is then connected and is close to the union $\gamma_{i n} \cup \gamma_{b}$. Let $\#(f, c):=\left|\pi_{0}\left(f^{-1}(c)\right)\right|$ for $c \in \mathbb{R}$. Near $\gamma^{\prime}$ we construct a taming function $f^{\prime}$ with $f^{\prime}\left(\gamma^{\prime}\right)=f(\gamma):=c$ as before. The following table summarizes the relation between $f^{\prime}$ and $f$, we fix $\varepsilon>0$ such that the level set of $f, f^{\prime}$ corresponding to the value $c \pm \varepsilon$ is a closed submanifold of $U, U^{\prime}$.

\begin{tabular}{l|l|l}
$\gamma_{i}$ attractive & $\#\left(f^{\prime}, c-\varepsilon\right)=\#(f, c-\varepsilon)-1$ & $h_{+}\left(U^{\prime}\right)=h_{+}(U)-1$ \\
$x_{0}$ positive & $\#\left(f^{\prime}, c+\varepsilon\right)=\#(f, c+\varepsilon)$ & $h_{-}\left(U^{\prime}\right)=h_{-}(U)$ \\
\hline$\gamma_{i}$ repulsive & $\#\left(f^{\prime}, c-\varepsilon\right)=\#(f, c-\varepsilon)$ & $h_{+}\left(U^{\prime}\right)=h_{+}(U)$ \\
$x_{0}$ negative & $\#\left(f^{\prime}, c+\varepsilon\right)=\#(f, c+\varepsilon)-1$ & $h_{-}\left(U^{\prime}\right)=h_{-}(U)-1$
\end{tabular}

This table implies that $f: U \rightarrow \mathbb{R}$ satisfies (iii) of Definition 4.8 if and only if the same is true for $f^{\prime}: U^{\prime} \rightarrow \mathbb{R}$. Because $\gamma$ was assumed to be connected it is not necessary to treat the case when each stable leaf of $x_{0}$ lies in an integral disc of $\xi$ with the same properties as $D_{1}^{\prime}, D_{m}^{\prime}$ above. By induction, this finishes the proof of (iii) of Definition 4.8 for $f: U \rightarrow \mathbb{R}$. 
We use a similar procedure to prove (6). We start with the case that $x_{0}$ is a hyperbolic singularity such that exactly one stable leaf of $x_{0}$ is a part of a cycle. After an isotopy of $S$ in a neighbourhood of $x_{0}$ we obtain a cycle $\gamma^{\prime}$ which contains one singularity less than $\gamma$. The following table summarizes the relations between $d_{+}, N_{s}, N_{-}, P_{s}$ for $U$ and $U^{\prime}$ if $x_{0}$ is positive or negative. When $x_{0}$ is negative, one has to consider two cases: Either (Case 1) the stable leaf of $x_{0}$ is the only stable leaf of a negative hyperbolic singularity intersecting the connected component of $\partial U$ or not (Case 2).

\begin{tabular}{l|ll} 
& $d_{+}\left(U^{\prime}\right)=d_{+}(U)+1$ & $N_{s}\left(U^{\prime}\right)=N_{s}(U)$ \\
& $N_{-}\left(U^{\prime}\right)=N_{-}(U)$ & $P_{S}\left(U^{\prime}\right)=P_{S}(U)-1$ \\
\hline$x_{0}$ is negative & $d_{+}\left(U^{\prime}\right)=d_{+}(U)$ & $N_{s}\left(U^{\prime}\right)=N_{s}(U)-1$ \\
$($ Case 1) & $N_{-}\left(U^{\prime}\right)=N_{-}(U)+1$ & $P_{S}\left(U^{\prime}\right)=P_{S}(U)$ \\
\hline$x_{0}$ is negative & $d_{+}\left(U^{\prime}\right)=d_{+}(U)$ & $N_{s}\left(U^{\prime}\right)=N_{s}(U)$ \\
$($ Case 2) & $N_{-}\left(U^{\prime}\right)=N_{-}(U)$ & $P_{S}\left(U^{\prime}\right)=P_{S}(U)$
\end{tabular}

The validity of (6) for $U$ follows from (6) for $U^{\prime}$. We may assume from now on that all stable and unstable leaves of all hyperbolic singularities on $\gamma$ are contained in $\gamma$. In particular, $N_{s}=P_{s}=0$ in the sequel.

Now assume that $x_{0} \in \gamma$ is a hyperbolic singularity such that both stable leaves are contained in exactly one integral disc of $\xi$ whose boundary is a cycle of $S(\xi)$ with well defined one-sided holonomy. In this case, the cycle on $S^{\prime}$ obtained by a controlled isotopy of $\xi$ consists of two connected components $\gamma_{1}^{\prime}, \gamma_{2}^{\prime}$. As before, we define functions $f_{i}^{\prime}: U_{i}^{\prime} \rightarrow \mathbb{R}, i=1,2$. The following table contains the relations between $d_{+}(U), d_{+}\left(U_{i}^{\prime}\right)$ and $N_{-}(U), N_{-}\left(U_{i}^{\prime}\right)$ with $i=1,2$.

\begin{tabular}{l|l}
$x_{0}$ is positive & $\begin{array}{l}d_{+}(U)=d_{+}\left(U_{1}^{\prime}\right)+d_{+}\left(U_{2}^{\prime}\right)-1 \\
\\
N_{-}(U)=N_{-}\left(U_{1}^{\prime}\right)+N_{-}\left(U_{2}^{\prime}\right)\end{array}$ \\
\hline$x_{0}$ is negative & $\begin{array}{l}d_{+}(U)=d_{+}\left(U_{1}^{\prime}\right)+d_{+}\left(U_{2}^{\prime}\right) \\
N_{-}(U)=N_{-}\left(U_{1}^{\prime}\right)+N_{-}\left(U_{2}^{\prime}\right)-1\end{array}$
\end{tabular}

It follows that (6) is true for $U$ because it is satisfied for $U_{1}^{\prime}, U_{2}^{\prime}$.

Now we assume that $x_{0}$ is a hyperbolic singularity such that one stable leaf is part of an internal cycle $\gamma_{i n}$ and the other one is part of a subcycle $\gamma_{b}$ which has well defined one-sided holonomy, contains $x_{0}$ and bounds a disc $D_{b}^{\prime}$ with $\gamma_{i n} \subset D_{b}$.

We isotope $S$ as before; in particular $\gamma^{\prime}$ is connected. The following table contains the relations between $d_{+}(U), d_{+}\left(U^{\prime}\right)$ and $N_{-}(U), N_{-}\left(U^{\prime}\right)$ depending on the sign of $x_{0}$. 


\begin{tabular}{l|l}
$x_{0}$ is positive & $\begin{array}{l}d_{+}\left(U^{\prime}\right)=d_{+}(U)+1 \\
N_{-}\left(U^{\prime}\right)=N_{-}\left(U^{\prime}\right)\end{array}$ \\
\hline$x_{0}$ is negative & $\begin{array}{l}d_{+}\left(U^{\prime}\right)=d_{+}(U) \\
N_{-}\left(U^{\prime}\right)=N_{-}(U)\end{array}$
\end{tabular}

As above, this shows (6) for the neighbourhood $U$ of $\gamma$ and $f: U \rightarrow \mathbb{R}$ has the desired properties.

This finishes the first step in the construction of a taming function on a neighbourhood of $\Sigma_{0}$. If all components of $\partial U$ are transverse to $S(\xi)$, then $U_{0}:=U$ and $f$ tames $S(\xi)$ on $U_{0}$. Otherwise we proceed as follows.

Assume we have constructed a taming function $f: U \rightarrow \mathbb{R}$ and $\Gamma \subset \partial U$ is a closed leaf of $S(\xi)$ with trivial holonomy. By construction the holonomy is potentially attractive on the side of $\Gamma$ which is contained in $U$. Then there is a cylinder $S^{1} \times(0,1) \subset S$ such that $S(\xi)$ corresponds to the foliation by the first factor and $\bar{C}$ consists of two cycles $\gamma_{0}, \gamma_{1}$ such that $\gamma_{0} \subset U$ and $\gamma_{1}$ lies in the complement of $U$. We choose $C$ maximal among cylinders with these properties. Then $\gamma_{1}$ can not be a closed leaf with trivial holonomy. Therefore $\gamma_{1}$ belongs to one of the following classes.

(i) $\gamma_{1}$ is a negative elliptic singularity or a closed leaf such that the holonomy on the side which is not contained in $C$ is nontrivial and potentially repulsive. In this case it is easy to extend $f$ to a taming function on $U \cup \bar{C}$ such that (6) is satisfied.

(ii) $\gamma_{1}$ is a cycle containing hyperbolic singularities. If we did not yet define a taming function near $\gamma_{1}$, then we apply the above procedure to construct a taming function $g: V \rightarrow \mathbb{R}$ on a set $V$ with $U \cap V=\varnothing$. In particular, $V$ satisfies (6). We add a constant to $g$ to ensure that $\left.g\right|_{\gamma_{1}}>\left.f\right|_{\Gamma}$. Then we extend $g \cup f: U \cup V \rightarrow \mathbb{R}$ to a taming function on $U \cup V \cup C$. Note that $N_{-}(U \cup V \cup C)=N_{-}(U)+N_{-}(V)-1$. Therefore (6) holds for $U \cup V \cup C$.

After finitely many steps we have constructed a taming function on a neighbourhood $U_{0}$ of $\Sigma_{0}$ with the desired properties.

If there $\Sigma_{0}$ contains a birth-death type singularity $x$ only minor modifications are necessary. According to our genericity assumptions $x$ lies in the interior or the contact region or in the interior of the foliated region and since $x \in \Sigma_{0}$, this singularity can only lie in the interior of the foliated region. In order to construct the taming function we can first ignore $x$ and than modify the resulting function on a neighbourhood of $x$. 
The following lemma implies that the existence of a taming function on a neighbourhood $U$ of $\Sigma$ is a property which is stable under $C^{0}$-small perturbations of $\xi$ if $U$ is small enough. This lemma is the key ingredient in the proof of Theorem 4.1.

Lemma 4.10 Let $\Sigma_{0}$ be a path connected component of $\Sigma(S)$ and $\tilde{\Sigma}_{0}$ the union of all critical points in $\Sigma_{0}$ and discs tangent to $\xi$ which bound cycles in $\Sigma_{0}$. There is an embedded ball $W$ containing $\widetilde{\Sigma}_{0}$ in its interior and $\varepsilon>0$ such that for every confoliation $\xi^{\prime}$ on $M$ which is $\varepsilon$-close (in the $C^{0}$-topology) to $\xi$ there is a confoliation $\xi_{c}^{\prime}$ on $\mathbb{R}^{3}$ which is transverse to the fibers of $\mathbb{R}^{3} \rightarrow \mathbb{R}^{2}$ and complete as connection together with an embedding

$$
\varphi:\left(W,\left.\xi^{\prime}\right|_{W}\right) \rightarrow\left(\mathbb{R}^{3}, \xi_{c}^{\prime}\right)
$$

such that $\varphi_{*}\left(\xi^{\prime}\right)=\xi_{c}^{\prime}$ and $\left.\xi^{\prime}\right|_{W}$ is tight.

Proof The integral discs which bound cycles depend continuously on the cycle because the integral discs are uniquely determined. On $\widetilde{\Sigma}_{0}$ we define an equivalence relation as follows: $x \sim y$ for $x, y \in \widetilde{\Sigma}_{0}$ if and only if there is a piecewise smooth path in $\widetilde{\Sigma}_{0}$ tangent to $\xi$ which connects $x$ and $y$.

The space $T:=\widetilde{\Sigma}_{0} / \sim$ should be thought of as a directed graph: Discs bounding singular cycles and closed leaves with nontrivial holonomy correspond to vertices while edges of $T$ correspond to families of integral discs of $\xi$ which bound a maximal connected cycle in $\Sigma_{0}$. (Because a disc in $\widetilde{\Sigma}_{0}$ may be part of a bigger disc in $\widetilde{\Sigma}_{0}$, a point in $T$ does not correspond to a unique cycle of $S(\xi)$ in general. This happens for example in Figure 12.) The orientation of an edge is induced by the coorientation of $\xi$. The space $T$ is a connected tree because $\widetilde{\Sigma}_{0}$ is connected and $S$ is a sphere. We embed $T$ in the $y, z$-plane in $\mathbb{R}^{3}$ such that $d z$ is consistent with the orientation of the edges of $T$.

Let $\mathcal{L}$ be the foliation on $\mathbb{R}^{3}$ by straight lines parallel to the $x$-axis and $\mathcal{Z}$ the foliation by planes parallel to the $x, y$-plane. We replace $T$ by a family of discs tangent to $\mathcal{Z}$ : For each vertex of $T$ we choose a collection of discs $D_{i}$ such that

- each $D_{i}$ is tangent to the leaf of $\mathcal{Z}$ containing the vertex and

- $\bigcup_{i} D_{i}$ is diffeomorphic to the union of integral discs in $M$ which bound the corresponding cycle in $M$ and $\bigcup_{i} D_{i}$ intersects the original tree $T$ in exactly one point.

Then we connect the discs which correspond to vertices of $T$ by families of discs tangent to $\mathcal{Z}$ as prescribed by the edges of $T$, ie by the configuration of integral discs 
in $M$. This is done in such a way that outside of a small neighbourhood of the discs which correspond to vertices of the tree each leaf of $\mathcal{L}$ intersects at most one disc and this intersection is connected. (In the presence of some configurations of critical points on cycles in $\Sigma_{0}$, eg two hyperbolic singularities on a cycle $\gamma \subset \Sigma_{0}$ such that each stable leaf of one singularity is an unstable leaf of the other, it may impossible these requirements near the largest integral disc containing $\gamma$.)

So far we have obtained an embedding $\varphi_{0}: \tilde{\Sigma}_{0} \rightarrow \mathbb{R}^{3}$ with $\varphi_{0 *}(\xi)=\mathcal{Z}$ and the Legendrian foliation $\varphi_{0 *}^{-1}(\mathcal{L})$ on $\tilde{\Sigma}_{0}$. We extend this foliation to a Legendrian foliation $\mathcal{L}_{0}$ on an open neighbourhood $\widetilde{\Sigma}$ of $\tilde{\Sigma}_{0}$ and we extend the embedding $\varphi_{0}$ such that the extended Legendrian foliation is mapped to $\mathcal{L}$, the extension of $\varphi_{0}$ is the desired embedding $\varphi: \widetilde{\Sigma} \rightarrow \mathbb{R}^{3}$ but we still have to fix the right domain and the neighbourhood $W$.

For this we choose $\tilde{\Sigma}$ such that the intersection of each leaf of $\mathcal{L}$ with $\varphi(\widetilde{\Sigma})$ is connected and $\varphi_{*}(\xi)$ is transverse to $\partial_{z}$. By construction $\varphi_{*}(\xi \mid \tilde{\Sigma})$ is the kernel of the $1-$ form $\alpha=d z+f(x, y, z) d y$ with $\partial_{x} f \geq 0$ and $f \equiv 0$ on $\widetilde{\Sigma}_{0}$.

By extending $f$ to a function on $\mathbb{R}^{3}$ we can extend $\alpha$ to a 1 -form $\alpha_{c}$ on $\mathbb{R}^{3}$ whose kernel is a confoliation $\xi_{c}$ with the desired properties: If we extend $f$ to a function on $\mathbb{R}^{3}$ with $\partial_{x} f \geq 0$ and $f \equiv 0$ for $|z|$ big enough, then $\xi_{c}$ is a complete connection.

For each plane field $\zeta$ on $\varphi(\widetilde{\Sigma})$ such that $\zeta$ is transverse to $\partial_{z}$ we define a foliation $\mathcal{L}(\zeta)$ which is tangent to the projection of $\partial_{x}$ to $\zeta$ along $\partial_{z}$. There is a neighbourhood $W \subset M$ of $\widetilde{\Sigma}_{0}$ and $\varepsilon>0$ with the following properties:

- $\partial W$ is piecewise smooth and never tangent to $\partial_{x}$.

- If $\xi^{\prime}$ is $\varepsilon$-close to $\xi$, then $\varphi_{*}(\xi)$ is transverse to $\partial_{z}$.

- For every plane field $\xi^{\prime}$ which is $\varepsilon$-close to $\xi$ there is an open set $W^{\prime}$ with $\widetilde{\Sigma}_{0} \subset W \subset W^{\prime} \subset U$ such that the intersection of $\varphi\left(W^{\prime}\right)$ with leaves of $\mathcal{L}\left(\varphi_{*}\left(\xi^{\prime}\right)\right)$ is connected.

We can choose $W$ to be an embedded ball since its image under $\varphi$ is a neighbourhood of a tree were the edges correspond to families of embedded disc while the vertices correspond to union of discs identified at boundary points such that the resulting union of discs is simply connected.

This implies the claim of the lemma: If a confoliation $\xi^{\prime}$ is sufficiently close to $\xi$ in the $C^{0}$-topology, then we can extend $\varphi_{*}\left(\left.\xi^{\prime}\right|_{W}\right)$ by extending (as above) the confoliation $\varphi_{*}\left(\left.\xi\right|_{W^{\prime}}\right)$ along leaves of a foliation $\mathcal{L}^{\prime}$ of $\mathbb{R}^{3}$ by lines transverse to the planes $\{x=$ const. $\}$ and which coincides with $\mathcal{L}$ outside of $\varphi(\tilde{\Sigma})$. Thus we have found a confoliation $\xi_{c}^{\prime}$ on $\mathbb{R}^{3}$ with the desired properties. 
The statement about the tightness of $\left.\xi^{\prime}\right|_{W}$ follows from Proposition 2.5. Note that we can choose $\partial_{x} f>0$ outside of $W$. Therefore no integral disc intersects the complement of $W$.

Next we show that the taming functions which we have constructed on pieces of $S$ in Lemma 4.9 can be combined to obtain a taming function on a given generically embedded sphere.

Proposition 4.11 If $(M, \xi)$ is tight and $S$ is an embedded sphere such that $S(\xi)$ has isolated singularities which are either nondegenerate or of birth-death type, then $S$ admits a taming function.

Proof We construct $f$ in a finite number of steps. By Lemma 4.9 we can cover the compact set $\Sigma(S)$ by a finite collection $\mathbb{U}_{0}$ of pairwise disjoint submanifolds $U_{1}, \ldots, U_{l}$ such that $\partial U_{i}$ is transverse to $S(\xi)$ and there are taming functions $f_{j}: U_{j} \rightarrow$ $\mathbb{R}$ for $i=1, \ldots, l$. Recall that

$$
d_{+}\left(U_{j}\right)=1-N_{-}\left(U_{j}\right)-P_{s}\left(U_{j}\right)-N_{s}\left(U_{j}\right)
$$

for all $j=1, \ldots, l$.

We define a partial order $\preceq$ on $\mathbb{U}_{0}$ as follows: $U_{j} \preceq U_{k}$ if and only if either $j=k$ or $U_{k}$ has a boundary component which bounds a disc in $S$ not containing $U_{k}$ and a leaf of the characteristic foliation coming from $U_{j}$ enters $U_{k}$ through this boundary component.

By definition, every cycle of $S(\xi)$ which intersects $U_{j}$ is completely contained in $U_{j}$. This implies that $U_{j} \preceq U_{k}$ and $U_{k} \preceq U_{j}$ if and only if $j=k$ and there is a set $U_{j} \in \mathbb{U}_{0}$ which is minimal with respect to $\preceq$. All connected components of $\partial U_{j}$ are transverse to $S(\xi)$, the characteristic foliation points outwards along the boundary and (7) implies $d_{+}\left(U_{j}\right)=1$.

Let $f_{j}$ be a taming function on $U_{j}$ and consider the basin $\overline{B\left(U_{j}\right)}$ of $U_{j}$. According to Lemma $3.4 \overline{B\left(U_{j}\right)}$ is covered by a Legendrian polygon $\left(Q_{j}, V_{j}, \alpha_{j}\right)$. We consider the following cases:

(o) $Q_{j}$ has more boundary components than $U_{j}$.

(i) A boundary component of $Q_{j}$ is mapped to an elliptic singularity respectively closed cycle and $\alpha_{j}\left(Q_{j}\right)$ is a neighbourhood of the elliptic singularity respectively a one-sided neighbourhood of the cycle.

(ii) A boundary component of $Q_{j}$ contains a positive pseudovertex $w$ such that $\alpha_{j}(w)$ has a stable leaf which is not contained in $\alpha_{j}\left(Q_{j}\right)$.

(iii) $\alpha_{j}$ identifies pseudovertices $w_{1}, \ldots, w_{l}$ and $\alpha_{j}\left(w_{i}\right)$ is negative for $i=1, \ldots, l$. 
According to Proposition 4.6 and the assumption on $d_{+},\left(Q_{j}, V_{j}, \alpha_{j}\right)$ belongs to at least one of these classes (o)-(iii). In the cases (i),(ii) and (iii) we assume in addition, that $\left(Q_{j}, V_{j}, \alpha_{j}\right)$ does not satisfy the hypotheses (o).

In order to simplify the presentation, we assume that $\alpha_{j}\left(Q_{j}\right)$ contains no singularities of birth-death type.

Case (o) Assume first that $\gamma_{j}$ is the stable leaf of a hyperbolic singularity $h_{j}$ such that $\gamma_{j}$ leaves $U_{j}$ and $h_{j}$ is a corner in a cycle $\eta$. This cycle is contained in one of the sets $U_{i(\eta)} \in \mathbb{U}_{0}$ with $i(\eta) \neq j$. Using the taming function $f_{i(\eta)}$ we extend $f_{j}$ to a taming function on a neighbourhood $U_{j}^{\prime}$ of $\gamma_{j} \cup U_{j} \cup U_{i(\eta)}$ (it may be necessary to add a sufficiently large constant to $f_{i(\eta)}$ ).

The extended function tames the characteristic foliation on its domain and $U_{j}^{\prime}$ can be chosen transverse to $S(\xi)$. By construction

$$
\begin{aligned}
N_{-}\left(U_{j}^{\prime}\right) & =N_{-}\left(U_{i(\eta)}\right) \\
P_{S}\left(U_{j}^{\prime}\right) & = \begin{cases}P_{S}\left(U_{i(\eta)}\right)-1 & \text { if } h_{j} \text { is positive, } \\
P_{S}\left(U_{i(\eta)}\right) & \text { if } h_{j} \text { is negative, }\end{cases} \\
N_{S}\left(U_{j}^{\prime}\right) & = \begin{cases}N_{S}\left(U_{i(\eta)}\right) & \text { if } h_{j} \text { is positive, } \\
N_{S}\left(U_{i(\eta)}\right)-1 & \text { if } h_{j} \text { is negative }\end{cases}
\end{aligned}
$$

This implies $d_{+}\left(U_{j}^{\prime}\right)=1-N_{-}\left(U_{j}^{\prime}\right)-P_{s}\left(U_{j}^{\prime}\right)-N_{s}\left(U_{j}^{\prime}\right)$.

In the following cases we consider a fixed connected component $\Gamma \subset \partial Q_{j}$.

Case (i) $\alpha_{j}(\Gamma)$ is an elliptic singularity (and $\alpha_{j}\left(Q_{j}\right)$ is a neighbourhood of $x$ ) or $\alpha_{j}(\Gamma)$ is a cycle and $\alpha_{j}\left(Q_{j}\right)$ is a one-sided neighbourhood of that cycle.

Let us start with the case when $\alpha_{j}(\Gamma)$ is an elliptic singularity. Because it is attractive, it must be negative and it is contained in $U_{i(\Gamma)}$ with $i(\Gamma) \neq j$. One can easily extend $f_{j}$ to a taming function on the union $U_{j}^{\prime}$ of $U_{j} \cup U_{i(\Gamma)}$ with all leaves passing through $\Gamma$. Obviously (7) holds for $U_{j}^{\prime}$.

If $\alpha_{j}(\Gamma)$ is a closed leaf or a cycle, then $\alpha_{j}(\Gamma)$ belongs to one of the sets $U_{i(\Gamma)}$ with $i(\Gamma) \neq j$. After eventually adding a constant to the taming function on $U_{i(\Gamma)}$ one obtains a taming function on the union of leaves of $S(\xi)$ exiting $U_{j}$ through $\Gamma$ with $U_{j}$ and $U_{i(\Gamma)}$. As before we denote the new domain by $U_{j}^{\prime}$. From

$$
\begin{aligned}
N_{-}\left(U_{j}^{\prime}\right) & =N_{-}\left(U_{i(\Gamma)}\right)-1 \\
P_{S}\left(U_{j}^{\prime}\right) & =P_{s}\left(U_{i(\Gamma)}\right) \\
N_{S}\left(U_{j}^{\prime}\right) & =N_{S}\left(U_{i(\Gamma)}\right),
\end{aligned}
$$

it follows that $d_{+}\left(U_{j}^{\prime}\right)=1-U_{-}\left(U_{j}^{\prime}\right)-P_{s}\left(U_{j}^{\prime}\right)-N_{s}\left(U_{j}^{\prime}\right)$. 
Case (ii) Let $\eta$ be the stable leaf of $x$ which is not contained in $\alpha_{j}\left(Q_{j}\right)$. The $\alpha$-limit set of $\eta$ is contained in a set $U_{i(\eta)}$ while $x \in U_{i(x)}$. We obtain a taming function on the union of $U_{j}^{\prime}$ of $U_{j} \cup U_{i(\eta)} \cup U_{i(x)}$ with a neighbourhood of the stable leaves of $x$ (after adding a constant to $f_{i(x)}$ ).

Because $x$ is positive the requirements in the definition of taming functions are satisfied. Moreover, we can choose the domain $U_{j}^{\prime}$ of the taming function such that its the new boundary component is transverse to $S(\xi)$. The equality $d_{+}\left(U_{j}^{\prime}\right)=$ $1-N_{-}\left(U_{j}^{\prime}\right)-P_{S}\left(U_{j}^{\prime}\right)-N_{S}\left(U_{j}^{\prime}\right)$ follows from the fact that $x$ is positive and

$$
\begin{aligned}
N_{-}\left(U_{j}^{\prime}\right) & =N_{-}\left(U_{i(\eta)}\right) \\
P_{S}\left(U_{j}^{\prime}\right) & =P_{s}\left(U_{i(\eta)}\right) \\
N_{s}\left(U_{j}^{\prime}\right) & =N_{s}\left(U_{i(\eta)}\right) .
\end{aligned}
$$

Case (iii) Let $w_{1}, \ldots, w_{l} \in \Gamma$ be the pseudovertices which are identified. Let us assume for simplicity that all hyperbolic singularities on $\sigma:=\gamma_{w_{1} \ldots w_{l}}$ are images of pseudovertices (the proof without this assumption is essentially the same, but the notation is more complicated).

The cycle $\sigma:=\gamma_{w_{1} \ldots w_{l}}$ is contained in $U_{i(\eta)} \in \mathbb{U}_{0}$ and we denote the stable leaves of the pseudovertices on $\sigma$ which are not part of $\sigma$ by $\eta_{1}, \ldots, \eta_{l}$. Let $U_{j}^{\prime}$ be the union of $U_{j} \cup U_{i(\sigma)}$ with neighbourhoods of $\eta_{1}, \ldots, \eta_{l}$. No other stable leaves of hyperbolic singularities on $\sigma$ enter $U_{i(\sigma)}$ and all pseudovertices on $\sigma$ are negative. After we add a sufficiently big constant to $f_{i(\eta)}$ we obtain a taming function $f_{j}^{\prime}$ on $U_{j}^{\prime}$. The following relations imply (6):

$$
\begin{aligned}
N_{-}\left(U_{j}^{\prime}\right) & =N_{-}\left(U_{i(\sigma)}\right) \\
P_{S}\left(U_{j}^{\prime}\right) & =P_{S}\left(U_{i(\sigma)}\right) \\
N_{S}\left(U_{j}^{\prime}\right) & =N_{S}\left(U_{i(\sigma)}\right)-1 .
\end{aligned}
$$

Finally, we treat the assumption that there is not birth-death type singularity. Assume that in one of the steps above, we encounter a birth-death type singularity $x$. Then $x$ is contained in a set $U_{i(x)}$ from $\mathbb{U}_{0}$. In an intermediate step we extend $f$ to the union $U_{j}^{\text {int }}$ of $U \cup U_{i(x)}$ with the leaves of $S(\xi)$ which connect $U_{i(x)}$ to $U$. Then we continue as before with $U_{j}^{\text {int }}$ instead of $U_{j}$.

At this point, we remove $U_{j}$ together with all $U_{i}$ which are contained in $U_{j}^{\prime}$ from the collection $\mathbb{U}_{0}$ and we add $U_{j}^{\prime}$. This yields a new collection of subsets $\mathbb{U}_{1}$ such that each domain in $\mathbb{U}_{1}$ admits a taming function. Notice that the number of sets in $\mathbb{U}_{1}$ is strictly smaller than the number of sets in $\mathbb{U}_{0}$.

We iterate the procedure after replacing $\mathbb{U}_{0}$ with $\mathbb{U}_{1}$. After finitely many steps we obtain a taming function on $S$. 
So far we have established the existence of a taming function on embedded spheres such that $S(\xi)$ has only nondegenerate or birth-death type singularities. Now we consider an embedding of $S^{2} \times[0,1]$ in $M$ and a $C^{0}$-approximation of $\xi$ by a confoliation $\xi^{\prime}$.

Proposition 4.12 There is a $C^{0}$-neighbourhood of $\xi$ such that for every confoliation $\xi^{\prime}$ in that neighbourhood $S_{t}\left(\xi^{\prime}\right)$ admits a taming function for all $t \in[0,1]$ if $S_{t}$ is generic with respect to $\xi^{\prime}$ for all $t$.

If $\xi^{\prime}$ is a contact structure, then $S_{t}\left(\xi^{\prime}\right)$ admits a taming function which is strictly increasing along all leaves of $S_{t}\left(\xi^{\prime}\right)$.

Proof We show that if $\xi^{\prime}$ is close enough to $\xi$ in the $C^{0}$-topology and $S_{t}\left(\xi^{\prime}\right)$ has only nondegenerate singularities or singularities of birth death type, then the iteration process used for the construction of a taming function in Proposition 4.11 can be carried out to yield a taming function for $S_{t}\left(\xi^{\prime}\right)$ for each $t \in[0,1]$. For this we first reconsider the proof of Proposition 4.11.

Let $\Sigma$ be the union of all singular points, closed leafs and cycles of $S_{t}(\xi)$ (for all $t \in[0,1])$. We cover $\Sigma$ by open subsets $U \subset M$ which have the following properties:

(i) $\overline{U \cap S_{t}}$ is a submanifold of $S_{t}$ with piecewise smooth boundary such that each smooth segment of $\partial U$ is transverse to $S_{t}(\xi)$ for some $t \in[0,1]$. Since this is an open condition we may assume that $U \cap S_{t}$ has this transversality property for all $t \in[0,1]$ such that $S_{t} \cap U$ is not empty.

(ii) For each $U$ there is a subset $W(U) \subset M$ containing $U$ such that $W(U)$ is a ball with the stability property from Lemma 4.10 for $\varepsilon(U)>0$.

Since $\Sigma \subset S^{2} \times[0,1]$ is compact we need only finitely many sets $U_{i}, i=1, \ldots, l$ which are contained in balls $W_{i}$ such that $\left.\xi^{\prime}\right|_{W_{i}}$ is tight if $\xi^{\prime}$ is $\varepsilon_{i}$-close to $\xi$ with $\varepsilon_{i}>0$. Let $0<\varepsilon^{\prime}<\varepsilon_{i}$ for all $i=1, \ldots, l$.

If $\xi^{\prime}$ is $\varepsilon^{\prime}$-close to $\xi$, then by Lemma 4.10 the characteristic foliation $S_{t}\left(\xi^{\prime}\right), t \in[0,1]$, admits a taming function on $U_{i} \cap S_{t}$ if $S_{t}\left(\xi^{\prime}\right)$ is a generic family of characteristic foliations.

In Proposition 4.11 we connected sets $U_{i} \cap S_{t}$ by leaves of the characteristic foliation of $S_{t}(\xi)$. All these leaves intersect $\partial U_{i} \cap S_{t}$ transversely and connect different sets $U_{i} \cap S_{t}$. Hence there is $\varepsilon^{\prime \prime}>0$ with the property that if $\xi^{\prime}$ is $\varepsilon^{\prime \prime}$-close to $\xi$, then leaves of $S_{t}\left(\xi^{\prime}\right)$ have the same property (eg no new singular points or cycles appear outside of $\bigcup_{i} U_{i}$ ).

Therefore we can carry out the first step of the proof of Proposition 4.11 to obtain taming functions on a new collection of submanifolds. Now we continue with the 
iteration process. At each step of the iteration we make $\varepsilon^{\prime \prime}$ smaller. Since there is only a finite number of sets $U_{i}$ the iteration terminates after finitely many steps. We have obtained a taming function for $S_{t}\left(\xi^{\prime}\right)$ for all $t \in[0,1]$ and choose $\varepsilon>0$ to be smaller than any $\varepsilon^{\prime \prime}$ appearing in the iteration steps.

Note that on each of the sets $U_{i} \cap S_{t}$ the number of singular points or closed leaves can change when $\xi$ is $C^{0}$-approximated by a confoliation $\xi$, but we know from Lemma 4.10 that on $U_{i} \cap S_{t}$ there is a taming function for $S_{t}(\xi)$ provided that $S_{t}\left(\xi^{\prime}\right)$ is generic and $\xi^{\prime}$ is sufficiently close to $\xi$ (with respect to the $C^{0}$-topology on the space of plane fields on $M$.) Proposition 4.12 enables us to prove the following theorem.

\subsubsection{Proof of Theorem 4.1 For the proof of Theorem 4.1 we combine the results} from the previous sections with results from [17].

Proof of Theorem 4.1 Let $B \subset B_{1} \subset M$ be an embedded closed ball in a manifold $M$ with a tight confoliation $\xi$. We assume that the interior of $B_{1}$ contains points where $\xi$ is a contact structure since otherwise Theorem 4.1 follows immediately from Lemma 4.10. Moreover, we assume that $\partial B_{1}$ is generic.

Let $B_{0}$ be a ball in the contact region whose characteristic foliation has exactly two singular points and the leaves of the characteristic foliation connect the two singularities. The existence of such a ball follows from the fact that every contact structure is locally equivalent to the standard contact structure $\operatorname{ker}(d z+x d y)$ on $\mathbb{R}^{3}$. Moreover, there is an open neighbourhood of $\left.\xi\right|_{B_{0}}$ such that every confoliation in this neighbourhood is tight on $B_{0}$. Let $\varepsilon>0$ be the constant from Proposition 4.12.

We fix an identification $B_{1} \backslash \stackrel{\circ}{B}_{0} \simeq S^{2} \times[0,1]$ such that $\partial B_{i}=S_{i}, i=0,1$ such that $S_{t}(\xi)$ is a generic 1-parameter family of characteristic foliations. Assume that $\xi^{\prime}$ is a contact structure on $M$ which is $\varepsilon / 2$ close to $\xi$ with respect to the $C^{0}$-topology.

In order to ensure that $S_{t}\left(\xi^{\prime}\right)$ is a generic 1-parameter family of foliations, we push forward $\xi^{\prime}$ by a diffeomorphism $\varphi$ of $M$ with $\varphi\left(\partial B_{i}\right)=\partial B_{i}$ which is so close to the identity in the $C^{1}$-topology, that $\varphi_{*}\left(\xi^{\prime}\right)$ is $\varepsilon$-close to $\xi$ in the $C^{0}$-topology. From now on we will write $\xi^{\prime}$ instead of $\varphi_{*}\left(\xi^{\prime}\right)$. According to Proposition 4.12 the characteristic foliations $S_{t}\left(\xi^{\prime}\right)$ admits a taming function for all $t \in[0,1]$.

Recall that an embedded surface in a contact manifold is called convex if there is a vector field transverse to the surface such that the flow of the vector field preserves the contact structure. According to [16] convexity is a $C^{\infty}$-generic property on orientable surfaces, so we may assume that $\partial B_{0}$ and $\partial B_{1}$ are convex with respect to $\xi^{\prime}$.

We will show that $\xi^{\prime}$ can be isotoped on $S^{2} \times[0,1]$ relative to the boundary such that all leaves of the product foliation on $S^{2} \times[0,1]$ become convex with respect to the 
isotoped contact structure. Since $\partial B_{0}$ is convex and $\xi^{\prime}$ is tight on a neighbourhood of $\partial B_{0}$ this implies that $\left.\xi^{\prime}\right|_{B}$ is tight by Theorem 2.19 in [17] (and the gluing result in [7]).

In order to prove the existence of the desired isotopy of $\xi^{\prime}$ we use the following lemma. Our formulation is a slight modification of Lemma 2.17 in [17] in the case $F \simeq S^{2}$.

Lemma 4.13 Let $\left(M, \xi^{\prime}\right)$ be a contact manifold. Assume that the characteristic foliation on each sphere $S_{t}$ from the family $S^{2} \times[0,1] \subset M$ admits a taming function and $S_{0}, S_{1}$ are convex. Then there is a contact structure $\xi^{\prime \prime}$ such that

- $\xi^{\prime}$ and $\xi^{\prime \prime}$ are isotopic relative to the boundary and

- the characteristic foliation of $\xi^{\prime \prime}$ on $S_{t}$ has exactly $\chi(S)=2$ singular points and $S_{t}$ is convex with respect to $\xi^{\prime \prime}$ for all $t \in[0,1]$.

The original statement of Giroux of this lemma contains tightness as an assumption. However the proof of Lemma 2.17 of [17] requires only properties of the characteristic foliation on $S_{t}, t \in[0,1]$ which follow from the existence of taming functions.

More specifically, the proof of Lemma 2.17 in [16] yields a proof of Lemma 4.13 after the following modification: As we have already explained we may assume that the characteristic foliation of $\xi^{\prime}$ on $S_{t}$ can also be tamed for all $t \in[0,1]$ by Proposition 4.12. Moreover, because $\xi^{\prime}$ is a contact structure, the taming functions are strictly increasing along leaves of the characteristic foliation. Therefore the following statements hold:

(1) There is no closed cycle on $S \times\{t\}, t \in[0,1]$.

(2) The graph $\Gamma_{t}^{+}\left(\Gamma_{t}^{-}\right)$on $F \times\{t\}$ formed by positive (negative) singular points and stable (unstable) leaves of positive (negative) hyperbolic singularities is a tree.

Using these two observations one obtains a proof of Lemma 4.13 from the proof of Lemma 2.17 in [17].

\section{Tight confoliations violating the Thurston-Bennequin in- equalities}

In this section we describe a construction of tight confoliations starting from a tight contact structure $\xi$ on a 3 -manifold whose boundary contains two incompressible tori $T_{0}, T_{1}$ such that $T_{i}(\xi), i=0,1$, is linear. Using this construction and the classification of tight contact structures on $T^{2} \times[0,1]$ from [17] we obtain a tight confoliation $\xi^{\prime}$ 
on $T^{3}$ and a torus $T \subset T^{3}$ such that $e\left(\xi^{\prime}\right)[T] \neq 0$, ie $\xi^{\prime}$ violates the ThurstonBennequin inequalities.

The results of this section indicate that tightness (as defined in Definition 1.3) is a much weaker condition for confoliations compared to the rigidity of tight contact structures or foliations without Reeb components.

Theorem 5.1 Let $\xi$ be a tight confoliation on $M$ and $T_{0}, T_{1} \subset \partial M$ two incompressible tori such that $\xi$ is a contact structure near $T_{i}$ and $T_{i}(\xi), i=0,1$ is linear. Let $\psi: T_{0} \rightarrow T_{1}$ be an orientation preserving diffeomorphism, where $T_{0}$, respectively $T_{1}$, is cooriented by an outward, respectively inward, pointing normal vector field.

The manifold $M^{\prime}=M \cup T_{0} \times[0,1] / \sim$, where $\sim$ identifies $T_{0} \simeq T_{0} \times\{0\}$ respectively $T_{1} \simeq T_{0} \times\{1\}$ using the identity respectively $\psi$, carries a tight confoliation $\xi^{\prime}$ such that $\left.\xi^{\prime}\right|_{M^{\prime} \backslash T_{0} \times[0,1]}$ is isomorphic to $\left.\xi\right|_{M \backslash T_{0} \cup T_{1}}$.

Proof Because the characteristic foliation on $T_{0}$ is linear we may assume that $\xi$ is defined by $\alpha=\cos \left(t+\beta_{0}\right) d x-\sin \left(t+\beta_{0}\right) d y$ for coordinates $x, y, t$ on a collar $U_{0}=T_{0} \times(-\varepsilon, 0], \varepsilon>0$, such that $x, y$ are coordinates on the first factor while $t$ corresponds to the second factor (cf [16]), $\beta_{0} \in \mathbb{R}$ is a constant and $\partial_{x}, \partial_{y}, \partial_{t}$ induces the orientation of $M$.

Now let $\rho:(-\varepsilon, 0] \rightarrow(-\varepsilon, 0]$ be a smooth homeomorphism which is a diffeomorphism on $(-\varepsilon, 0)$ such that all derivatives of $\rho$ at $t=0$ vanish. Moreover, we assume that $\rho(t)=t$ for all $t \in(-\varepsilon,-\varepsilon / 2)$. Then the map $(x, y, t) \longmapsto\left(x, y, \rho^{-1}(t)\right)$ extends to a diffeomorphism of $M \backslash T_{0}$ and it maps $\xi$ to a confoliation $\xi^{\prime \prime}$ which coincides with $\xi$ outside of $U_{0}$ and is defined by $\alpha_{0}^{\prime \prime}=\cos (\rho(t)+\beta) d x-\sin (\rho(t)+\beta) d y$ on $U_{0}$. In particular, $\xi^{\prime \prime}$ extends to a smooth confoliation on $M$. We now extend this confoliation to a confoliation on $M \cup T_{0} \times[0,1 / 2] / \sim$ where $\sim$ identifies $T_{0} \subset \partial M$ with $T_{0} \times\{0\}$ using the identity map.

On $T_{0} \times[0,1 / 2]$ consider the foliation $\xi_{0}$ defined by

$$
\alpha_{0}=d \tau+(1 / 2-\tau)\left(\cos \left(\beta_{0}\right) d x-\sin \left(\beta_{0}\right) d y\right)
$$

where we use the same coordinates $x, y$ on $T_{0}$ as before, the coordinate $\tau$ corresponds to the second factor of $T_{0} \times[0,1 / 2]$. The characteristic foliation on $T_{0} \times\{0\}$ is linear and coincides with $T_{0}(\xi)$.

Hence we obtain a smooth confoliation $\xi^{\prime \prime}$ on $M \cup T_{0} \times[0,1 / 2] / \sim$ after identifying $T_{0} \subset \partial M$ with $T_{0} \times\{0\}$ (the differentiable structure in directions transverse to $\partial M$ is such that $\partial_{t}$ together with $\partial_{\tau}-(1 / 2-\tau)^{-1}\left(\cos (\beta) \partial_{x}-\sin (\beta) \partial_{y}\right)$ is smooth on a neighbourhood of $T_{0}$ ). The coorientation of $\xi^{\prime \prime}$ is given by $\alpha_{0}$ and $\alpha$. Note that 
$T_{0} \times\{1 / 2\}$ is a closed leaf of $\xi^{\prime}$ which is cooriented by $\partial_{\tau}$, ie by an outward pointing vector field.

A similar construction can be applied to $T_{1}$. So we obtain an oriented confoliation $\xi^{\prime \prime}$ on

$$
M^{\prime \prime}=\left(T_{0} \times[0,1 / 2]\right) \cup_{T_{0} \times\{0\}} M \cup_{T_{1} \times\{1\}}\left(T_{1} \times[1 / 2,1]\right)
$$

such that $T_{0}^{\prime \prime}=T_{0} \times\{1 / 2\}$ and $T_{1}^{\prime \prime}=T_{1} \times\{1 / 2\}$ are closed leaves in the boundary of $M^{\prime \prime}$ and $T_{0}^{\prime \prime}$ respectively $T_{1}^{\prime \prime}$ are cooriented by a vector field which points out of $M^{\prime \prime}$ respectively into $M^{\prime \prime}$.

Identifying $T_{0}^{\prime \prime}$ with $T_{1}^{\prime \prime}$ using $\psi$ one obtains a plane field on the resulting manifold $M^{\prime}$, but this plane field is only continuous in general. In order to ensure that the resulting plane field is smooth one modifies the foliations on $T_{0} \times[0,1 / 2]$ and $T_{1} \times[1 / 2,1]$ on a neighbourhood of $T_{0}^{\prime \prime}$ and $T_{1}^{\prime \prime}$ as follows.

For fixed generators $c, c^{\prime}$ of $\pi_{1}\left(T_{0}^{\prime \prime}\right)$ and small enough $\varepsilon>0$ the holonomy along $c, c^{\prime}$ is defined on $(1 / 2-\varepsilon, 1 / 2]$. Fix a smooth homeomorphism $\Phi:(1 / 2-\varepsilon, 1 / 2] \rightarrow$ $(1 / 2-\varepsilon, 1 / 2]$ such that all derivatives of $\Phi$ vanish at $1 / 2$ and which is the identity near $1 / 2-\varepsilon$. Conjugating the holonomy diffeomorphisms along $c, c^{\prime}$ with $\Phi \circ \Phi$, one obtains a foliation on $T_{0} \times[0,1 / 2]$ which coincides with the original foliation near $T_{0} \times\{0\}, T_{0}^{\prime \prime}$ is a closed leaf and along $T_{0} \times\{1 / 2\}$ the new foliation is $C^{\infty}$-tangent to the product foliation on $T_{0} \times[0,1 / 2]$.

Applying this modification to $T_{1} \times[1 / 2,1]$ we obtain a smooth confoliation $\xi^{\prime}$ on $M^{\prime}$. This manifold contains a layer $T_{0} \times[0,1]$ where $\xi^{\prime}$ is a foliation with a unique closed leaf $T_{1 / 2}:=T_{0} \times\{1 / 2\}$.

In order to prove that $\xi^{\prime}$ is tight we show that the contrary contradicts the tightness of $\xi$ on $M$. Let $\gamma \subset M^{\prime}$ be a Legendrian curve which bounds an embedded disc $D$ such that $\xi^{\prime}$ is nowhere tangent to $D$ along $\gamma$ and which violates the requirements of Definition 1.3. Because $\xi$ is tight, $\gamma$ must intersect the layer $T_{0} \times[0,1]$ which we added to $M$.

Note that $\gamma$ cannot intersect $T_{1 / 2}$, since otherwise the $\gamma$ is contained in $T_{1 / 2}$ and $\gamma$ bounds a disc $D^{\prime}$ in $T_{1 / 2}$ because both $T_{0}$ and $T_{1}$ were incompressible. The cycle $D \cup D^{\prime}$ is homologous to a union of embedded spheres in $M$, thus $e\left(\xi^{\prime}\right)\left[D \cup D^{\prime}\right]=0$ by Theorem 4.4.

Now we assume that $\gamma \cap T_{1 / 2}=\varnothing$. If $\gamma$ is contained in $T_{0} \times[0,1] \backslash T_{1 / 2}$, then $\gamma$ bounds a disc $D^{\prime}$ in the leaf of the foliation because all leaves of the foliation on $T_{0} \times[0,1]$ are either incompressible cylinders or planes. As before $e\left(\xi^{\prime}\right)\left[D \cup D^{\prime}\right]=0$.

It remains to treat the case when $\gamma$ intersects both the contact region and $T_{0} \times[0,1]$. All leaves of $\xi^{\prime}$ in $T_{0} \times[0,1] \backslash T_{1 / 2}$ are incompressible cylinders or planes which can 
be retracted into $M$. First, we show that there is a Legendrian isotopy of $\gamma$ such that the resulting curve is transverse to $T_{0} \cup T_{1}$. Because a similar isotopy will be used later we describe it in detail:

Fix a tubular neighbourhood $T_{0} \times(-\varepsilon, \varepsilon), \varepsilon>0$, of $T_{0}$ in $M^{\prime}$ and coordinates $x, y$ on $T_{0}, t$ on $(-\varepsilon, \varepsilon)$ such that $\xi^{\prime}$ is defined by

$$
\alpha=d x-f d y .
$$

By the construction of $\xi^{\prime}$ we may assume that $f$ depends only on $t$ and $f^{\prime} \leq 0$ since $\xi^{\prime}$ is a positive confoliation. Consider the projection pr: $T_{0} \times(-\varepsilon, \varepsilon) \rightarrow S^{1} \times(-\varepsilon, \varepsilon)$ such that the fibers are tangent to $\partial_{x}$ and hence transverse to $\xi^{\prime}$. Then $d \alpha_{0}$ is the pullback of $\omega=-f^{\prime}(z) d y \wedge d t$. Let $\hat{\gamma}$ be a segment of $\gamma$ which is contained in $T^{2} \times[0, \varepsilon]$ and whose endpoints do not lie on $T_{0} \times\{0\}$.

If $\hat{\gamma}$ is contained in the foliated part of $\xi^{\prime}$, then we isotope $\hat{\gamma}$ within its leaf such that the resulting curve is disjoint from $T_{0} \times\{0\}$ and the isotopy does not affect the curve on a neighbourhood of its endpoints.

Now assume that some pieces of $\hat{\gamma}$ are contained in the contact region of $\xi^{\prime}$. Then $\operatorname{pr}(\hat{\gamma})$ passes through $S^{1} \times(-\varepsilon, 0)$ where $\omega$ is nonvanishing. We choose an isotopy of the projection of $\hat{\gamma}$ which is fixed near the endpoints and the area of the region bounded by $\hat{\gamma}$ and each curve in the isotopy is zero. Then one obtains closed Legendrian curves when $\hat{\gamma}$ is replaced by horizontal lifts of curves of the isotopy (with starting point on $\gamma$ ).

Hence we may assume that $\gamma$ is transverse to $T_{0} \times\{0\}$ and $\gamma$ is decomposed into finitely many segments whose interior is completely contained in either the contact region or the foliated region of $\xi^{\prime}$.

Let $\gamma_{0} \subset \gamma$ be an arc with endpoints in the contact region of $\xi$ such that $\gamma_{0}$ contains exactly one subarc of $\gamma$ lying in the foliated region. Because $\gamma_{0}$ is embedded, its union with a segment of $T_{0}\left(\xi^{\prime}\right)$ bounds a compact half disc in a leaf tangent to $\xi^{\prime}$ and we can choose $\gamma_{0}$ such that the half disc does not contain any other segment of $\gamma$.

Now we isotope $\gamma_{0}$ relative to its endpoints such that after the isotopy this segment lies completely in the contact region of $\xi$. As above we deform $\operatorname{pr}\left(\gamma_{0}\right)$ through immersions such that the resulting arc $\widehat{\gamma}_{0}$ has the following properties:

- The integral of $\omega$ over the region bounded by $\hat{\gamma}_{0}$ and $\operatorname{pr}\left(\gamma_{0}\right)$ is zero and the same condition applies to every curve in the isotopy.

- $\hat{\gamma}_{0}$ is completely contained in $S^{1} \times(-\varepsilon, 0)$.

Then the horizontal lift of $\widehat{\gamma}_{0}$ can be chosen to have the same endpoints as $\gamma_{0}$ and we can replace $\gamma_{0}$ by this horizontal lift. The resulting curve is Legendrian isotopic to $\gamma$ 
but the number of pieces which lie in the foliated region has decreased by one. The segments of $\gamma$ in $T_{1} \times(1 / 2,1]$ can be treated in the same fashion.

After finitely many steps we obtain a Legendrian isotopy between $\gamma$ and a Legendrian unknot which lies completely in the interior of $M$. The resulting unknot still bounds a disc with the properties of $D$ in Definition 1.3 but it intersects the contact region. Therefore there is no disc $D^{\prime}$ with the properties from Definition 1.3. This contradiction shows that $\xi^{\prime}$ is tight.

The construction above should be compared with the following result by V Colin [8]: If $\xi$ is a contact structure on $M$ and $T \subset M$ an incompressible torus such that $T(\xi)$ is linear and the induced contact structure on the universal cover of $M \backslash T$ is tight, then $\xi$ is tight on $M$. In Theorem 5.1 we do not require tightness on the universal cover and we do not prescribe how $T_{0}$ and $T_{1}$ are identified.

Theorem 5.1 can be used to construct tight confoliations violating the ThurstonBennequin inequalities. For this let $M=T^{2} \times[0,1], T_{0}=T^{2} \times\{0\}$ and $T_{1}=T^{2} \times\{1\}$. According to Giroux [17] there is a unique tight contact structure $\xi$ on $T^{2} \times[0,1]$ such that

(i) the characteristic foliation on $\partial\left(T^{2} \times I\right)$ is a pair of linear foliations whose slope is 2 , respectively $1 / 2$, on $T_{0}$, respectively $T_{1}$, and

(ii) the obstruction for the extension of the vector fields which span the characteristic foliation on $\partial\left(T^{2} \times I\right)$ is Poincaré-dual to $(2,2) \in H_{1}\left(T^{2} ; \mathbb{Z}\right) \simeq \mathbb{Z}^{2}$.

Using Theorem 5.1 one obtains a tight confoliation on the manifold $M^{\prime}=T^{3}$ obtained by gluing two copies of $T^{2} \times[0,1]$ along the boundary. In order to show that the resulting confoliation $\xi^{\prime}$ violates inequality (b) of Theorem 1.6 and also to obtain an indication how Definition 1.3 could be improved, we study this confoliation in more detail. For this we will use the notation from the proof of Theorem 5.1.

Figure 15 shows the characteristic foliation of the contact structure $\xi$ on $T^{2} \times\{t\} \subset M$ for various $t \in[0,1]$ together with its orientation. The two curves in $T^{2} \times\{1 / 2\} \subset M$ where the characteristic foliation is singular represent the homology class $(2,2) \in$ $H_{1}\left(T^{2} ; \mathbb{Z}\right)$.

The trivialization of $\xi$ induced by the characteristic foliation on $T^{2} \times\{0,1\}$ extends to the complement of $M$ in $T^{3}$. The obstruction for the extension of the trivialization from $\partial M$ to $M$ is Poincaré-dual to $(2,2) \in H_{1}(M)$. Hence $e\left(\xi^{\prime}\right)$ is Poincare-dual to $(2,2,0) \in H^{1}\left(T^{2}\right) \oplus \mathbb{Z}$ where the second factor corresponds to the homology of the second factor of $T^{3} \simeq T^{2} \times S^{1}$. This means that $\xi^{\prime}$ violates the Thurston-Bennequin inequalities since these inequalities would imply $e\left(\xi^{\prime}\right)=0$. 


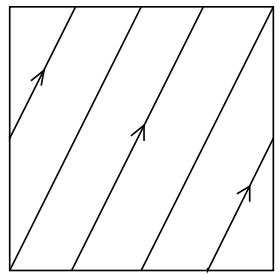

$t=0$

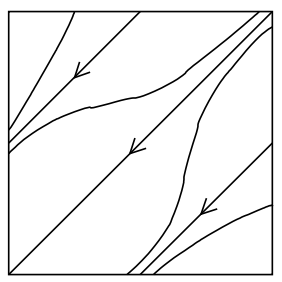

$t=2 / 3$

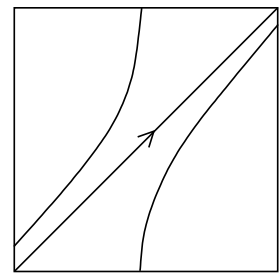

$t=1 / 6$

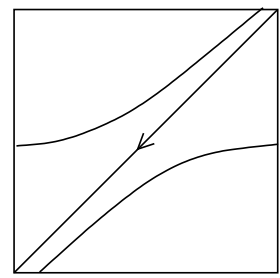

$t=5 / 6$

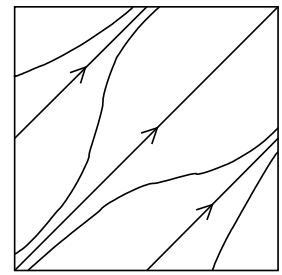

$t=1 / 3$

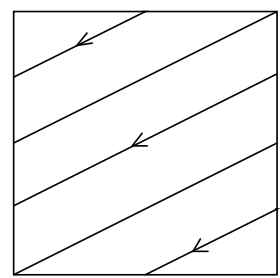

$t=1$

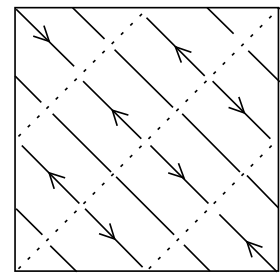

$t=1 / 2$

Figure 15

An example of a torus in $\left(T^{3}, \xi^{\prime}\right)$ which violates the Thurston-Bennequin inequality can be described explicitly. Let $T$ be the torus which is invariant under the $S^{1}$-action transverse to the fibers of $T^{2} \times S^{1} \rightarrow S^{1}$ and which intersects each fiber in a curve of slope -1 , hence this curve represents $(1,-1) \in H_{1}\left(T^{2}\right)$ when $T$ is suitably oriented.

It follows from the construction in the proof of Theorem 5.1 that $T\left(\xi^{\prime}\right)$ contains two closed Legendrian curves: One corresponds to the closed leaf of $\xi^{\prime}$ introduced in the proof of Theorem 5.1 while the other one corresponds to the Legendrian curves in Figure $15(t=1 / 2)$ of slope -1 . The latter Legendrian curve contains exactly four singularities of $T\left(\xi^{\prime}\right)$ and these singularities have alternating signs. Moreover $\xi^{\prime}$ is transverse to all tori $T^{2} \times\{t\}, t \in S^{1}$ except the closed leaf of $\xi^{\prime}$ and $T^{2} \times\{1 / 2\} \subset M$. This determines the $T\left(\xi^{\prime}\right)$ : Figure 16 shows a singular foliation homeomorphic to the one on $T$. We choose the orientation of $T$ such that $e(T)=-4$. In order to find an example of a surface with boundary which violates the inequality (c) from Theorem 1.6 it suffices to remove a small disc containing one of the elliptic singularities in $T$.

According to [10] every positive confoliation can be approximated (in the $C^{0}$-topology) by a contact structure. From this it follows that tightness is not an open condition in the space of confoliations with the $C^{0}$-topology. Actually $\xi^{\prime}$ can be approximated by contact structures which are $C^{\infty}$-close to $\xi^{\prime}$. This can be seen by going through the proofs of Theorem 2.4.1 and Proposition 2.5.1 in [10]: By construction the holonomy of the closed leaf on $T$ satisfies conditions which imply the conclusion of Proposition 2.5.1 


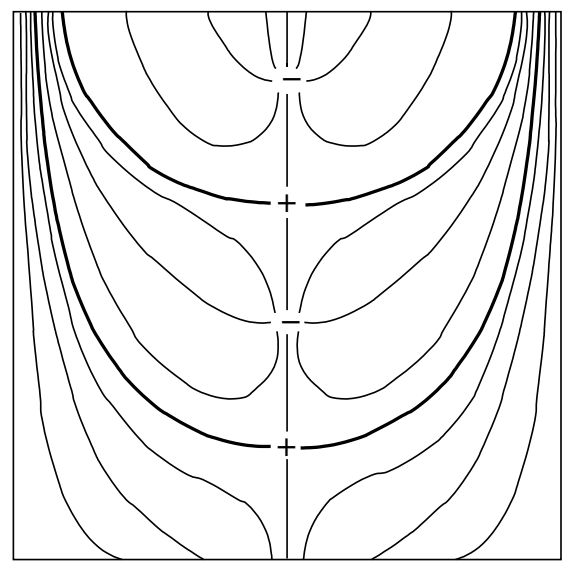

Figure 16

of [10] (despite of the fact that the infinitesimal holonomy is trivial). The main part of this proposition is stated in Lemma 6.3 together with an outline of the proof.

Thus tightness is not an open condition for confoliations in general. This answers Question 1 from the Section 3.7 in [10] (when tightness is defined as in Definition 1.3).

\section{Overtwisted stars}

In this section we introduce overtwisted stars. Their definition is given in the next section and it is motivated by the discussion of the confoliation $\left(T^{3}, \xi^{\prime}\right)$ in Section 5 . The absence of overtwisted stars in a tight confoliations implies all Thurston-Bennequin inequalities and we show that symplectically fillable confoliations do not admit overtwisted stars (in addition to the fact that they are tight).

\subsection{Overtwisted stars and the Thurston-Bennequin inequalities}

As we have already mentioned the point where the proof of the Thurston-Bennequin inequalities for tight confoliations fails is the following: Given an embedded surface $F$ and a tight confoliation $(M, \xi)$, there may be leaves of $F(\xi)$ which come from an elliptic singularity and accumulate on closed leaves $\gamma$ (or on quasi-minimal sets) of the characteristic foliation such that $\gamma$ is part of the fully foliated set of $\xi$. Even if all singular points on $\partial B(x)$ have the same sign it may be impossible to construct a disc from $B(x)$ which has the properties of the disc $D$ appearing in Definition 1.3.

This suggests the following definition of overtwisted stars on generically embedded surfaces $F$. 
Definition 6.1 An overtwisted star in the interior of a generically embedded compact surface $F \not S^{2}$ is the image of a Legendrian polygon $(Q, V, \alpha)$ with the following properties.

(i) $Q$ is homeomorphic to a disc and $\alpha(\partial Q)$ contains singularities of $F(\xi)$.

(ii) All singularities of $F(\xi)$ on $\alpha(\partial Q \backslash V)$ have the same sign. There is a single singularity in the interior of $\alpha(Q)$; it is elliptic and its sign is opposite to the sign of the singularities on $\alpha(\partial Q)$.

(iii) If $v \in V$ and $\gamma_{v}$ is a cycle, then $\gamma_{v}$ does not bound an integral disc of $\xi$ in $M$.

A confoliation is s-tight if it is tight such that no embedded surface contains an overtwisted star.

The torus shown Figure 16 contains two overtwisted stars. Note that the polygon is not required to be injective. Requirement (i) implies that either $V \neq \varnothing$ or $\alpha(\partial Q)$ contains an elliptic singularity of $F(\xi)$ and we may assume that this singularity is contained in $H(\xi)$ after a small perturbation of $S$. In particular, discs with the properties of $D$ in Definition 1.3 are not overtwisted stars.

If $\xi$ is a contact structure and $F \subset M$ is a generically embedded closed surface containing an overtwisted star $(Q, V, \alpha)$, then $\xi$ cannot be tight since $\xi$ is convex by the genericity assumption (therefore all $\gamma_{v}, v \in V$ are cycles) and has a homotopically trivial dividing curve (this terminology is standard in contact topology; because we shall not really use it we refer the reader to $[15 ; 16 ; 22])$. This argument does not apply when $F \simeq S^{2}$. Since the definition of tightness in Definition 1.3 can be applied efficiently to spheres and discs, the exceptional role of spheres in Definition 6.1 will not play a role.

The following theorem is proved using Eliashberg's strategy from [9] and Theorem 4.4.

Theorem 6.2 Let $(M, \xi)$ be an oriented s-tight confoliation which is not a foliation by spheres. Every compact embedded surface $F$ whose boundary is either empty or positively transverse to $\xi$ satisfies the following relations.

(a) If $F \simeq S^{2}$, then $e(\xi)[F]=0$.

(b) If $\partial F=\varnothing$ and $F \not S^{2}$, then $|e(\xi)[F]| \leq-\chi(F)$.

(c) If $\partial F \neq \varnothing$ is positively transverse to $\xi$, then $\operatorname{sl}(\gamma,[F]) \leq-\chi(F)$. 
Proof Claim (a) was already proved in Theorem 4.4. For the proofs of (b) and (c) we may assume that $F$ is a generic representative of the homology class $[F] \in H_{2}(M, \partial F)$ which is incompressible (this means that the map $\pi_{1}(F) \rightarrow \pi_{1}(M)$ induced by the inclusion $F \hookrightarrow M$ is injective). Recall that if $\partial F$ is positively transverse to $\xi$, then $F(\xi)$ points out of $F$ along $\partial F$ and

$$
\chi(F)-e(\xi)[F]=2\left(e_{-}-h_{-}\right)
$$

by Equation (4). If there is no negative elliptic singularity, then $-e(\xi)[F] \leq-\chi(F)$. If there is a negative elliptic singularity $x$, then we shall use the absence of overtwisted stars to eliminate $x$ without creating new negative elliptic singularities. Let $D_{x}$ be the maximal open disc in $F$ such that

- $\partial D_{x}=\bar{D}_{x} \backslash D_{x}$ is a cycle of $F(\xi)$ and

- $x$ is the only singularity of $F(\xi)$ in the interior of $D_{x}$.

Unless $\partial D_{x}=\varnothing$ there is an integral disc $D_{x}^{\prime}$ of $\xi$ whose boundary is $\partial D_{x}$ because $\xi$ is tight. Moreover, the intersection of the interior of $D_{x}^{\prime}$ with $F$ consists of homotopically trivial curves in $F$ (otherwise we get a contradiction to the incompressibility of $F$ ).

Thus we can cut $F$ using Lemma 3.7, Lemma 3.8 and Lemma 3.9 so that the resulting surface $F^{\prime}$ is the union of spheres and a surface which is diffeomorphic to $F$ and incompressible (as shown in the proof of Theorem 4.4, integral discs do not intersect $\partial F$ ). Because $e(\xi)[S]=0$ for embedded spheres $S$ we can ignore the spherical components and we denote the remaining surface by $F^{\prime}$. This surface satisfies $e(\xi)[F]=e(\xi)\left[F^{\prime}\right]$.

If we used Lemma 3.8 or Lemma 3.9, then we have reduced the number of negative elliptic singularities by one. Note that if we have applied Lemma 3.9, then $F^{\prime}$ might contain a circle of singularities. This means that $F^{\prime}$ is nongeneric near that circle. Since this circle is isolated from the rest of $F^{\prime}$ by closed leaves of $F^{\prime}(\xi)$ and the singularities on this circle do not contribute to $e(\xi)\left[F^{\prime}\right]$ or $\chi\left(F^{\prime}\right)$ we can pretend that $F^{\prime}$ is generic and continue to eliminate the remaining negative elliptic singularities.

If we used Lemma 3.7, then $F^{\prime}$ contains a negative elliptic singularity $x^{\prime}$ which lies in $H(\xi)$. In the following we shall denote $x^{\prime}$ again by $x$.

The basin of $x$ is covered by a Legendrian polygon $\left(Q^{\prime}, V^{\prime}, \alpha^{\prime}\right)$ on $F^{\prime}$. By the maximality property of $D_{x}$ the boundary of $Q^{\prime}$ is not mapped to a cycle of $F^{\prime}(\xi)$. If $\partial Q^{\prime}$ has more than one connected component, then there is a hyperbolic singularity $y$ on $\alpha^{\prime}\left(\partial Q^{\prime}\right)$ which is the corner of a cycle $\gamma_{y}$. If $y$ is negative, then we can eliminate the pair $x, y$.

Now assume that $y$ is positive. If $\gamma y$ does intersect $H(\xi)$, then we can perturb $F^{\prime}$ in a small neighbourhood of a point on the cycle such that $y$ is no longer part of a 
cycle after the perturbation. If $\gamma_{y}$ does not intersect $H(\xi)$, then we push a part of the cycle into $H(\xi)$ by an isotopy of $F^{\prime}$ without introducing new singularities of the characteristic foliation.

The isotopy is constructed as follows. Let $L$ be the maximal connected integral surface of $\xi$ which contains the cycle through $y$. We choose a simple curve $\sigma$ tangent to $\xi$ which connects the cycle to $H(\xi)$ and is disjoint from $F^{\prime}$. This curve can be chosen close to the stable leaf of $y$ which is connected to $x \in H(\xi)$. We choose a vector field $X$ tangent to $\xi$ with support in a small neighbourhood of $\sigma$ such that $\sigma$ is a flow line of $X$ and $F^{\prime}$ is transverse to $X$. We use the flow of $X$ to isotope $F^{\prime}$ such that all unstable leaves of $y$ are connected to $H(\xi)$ after the isotopy. Since $X$ is transverse to $F^{\prime}$ and tangent to $X$ the isotopy creates no new singular points of the characteristic foliation. Figure 17 shows $L$ together with a part of the intersection $F^{\prime} \cap L$. The curve $\sigma$ is represented by the thickened line while the shaded disc represents another part of $H(\xi)$ or nontrivial topology of $L$.

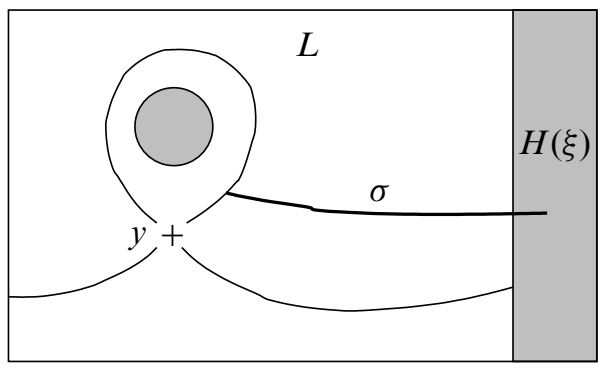

Figure 17

By this process we modified the basin of $x$. Note that there are finitely many hyperbolic singularities on $F$ and the procedure described above does not create new ones. Therefore finitely many applications of the entire procedure lead to a surface $F^{\prime \prime}$ with $e(\xi)[F]=e(\xi)\left[F^{\prime \prime}\right]$ such that the hyperbolic singularities of $F^{\prime \prime}(\xi)$ are also hyperbolic singularities of $F(\xi)$ and the basin of $x$ is homeomorphic to a disc. Also, the number of negative elliptic singularities did not increase and $F^{\prime \prime}$ is diffeomorphic to $F$, so $F \not S^{2}$.

The basin of $x$ is covered by a Legendrian polygon $\left(Q^{\prime \prime}, V^{\prime \prime}, \alpha^{\prime \prime}\right)$ on $F^{\prime \prime}$ such that $Q^{\prime \prime}$ is a disc and $\alpha^{\prime \prime}\left(Q^{\prime \prime}\right)$ is not an elliptic singularity or a cycle of $F^{\prime \prime}(\xi)$. If necessary, we eliminate all elements of $v^{\prime \prime} \in V$ with the property that $\gamma_{v^{\prime \prime}}$ is null homotopic in $F^{\prime \prime}$.

Now the absence of overtwisted stars implies that $\partial Q^{\prime \prime}$ contains a negative pseudovertex. By Lemma 3.5 we can isotope $F^{\prime \prime}$ to a surface containing less negative elliptic 
singularities than $F$ respectively $F^{\prime \prime}$. After finitely many steps we have eliminated all negative elliptic singularities. This finishes the proof of (c) and one of the inequalities in (b). The remaining inequality in (b) can be proved by eliminating all positive elliptic singularities.

\subsection{Overtwisted stars and symplectic fillings}

In this section we show that symplectically fillable confoliations do not admit overtwisted stars. In the proof we $C^{0}$-approximate a confoliation by another confoliation (cf Theorem 1.1). Several techniques used in the proof are adaptations of constructions in [10]. Other useful references are Petronio [28] (where the proofs of Proposition 2.5.1 c) and Lemma 2.5.3 from [10] are carried out) and Etnyre [14]. For later use we summarize the proof of a lemma used to show Theorem 1.1.

Lemma 6.3 [10, Proposition 2.5.1 c)] Let $\gamma$ be a simple closed curve in the interior of an integral surface $L$ of $\xi$. If $\gamma$ has sometimes attractive holonomy, then in every $C^{0}$-neighbourhood of $\xi$ there is a confoliation $\xi^{\prime}$ which

(i) is a contact structure on a neighbourhood of $\gamma$ and

(ii) coincides with $\xi$ outside a slightly larger neighbourhood.

Proof We only indicate the main stages of the construction. Fix a neighbourhood $V \simeq S_{x}^{1} \times[-1,1]_{y} \times[-1,1]_{z}$ and coordinates $x, y, z$ such that the foliation by the second factor is Legendrian, $S^{1} \times[-1,-1] \times\{0\} \subset L$ and $S^{1} \times\{(0,0)\}$ corresponds to $\gamma$. We assume that $\gamma$ has sometimes attractive holonomy. As shown in Lemma 2.1.1 of [28] the coordinates can be chosen such that

- $\xi$ is defined by the 1 -form $\alpha=d z+a(x, y, z) d x$ with $\partial_{y} a \leq 0$ and

- there are sequences $\zeta_{n}^{\prime}<0<\zeta_{n}$ converging to zero such that $a\left(x, 0, \zeta_{n}^{\prime}\right)<0<$ $a\left(x, 0, \zeta_{n}\right)$ for all $x$.

At this point we use the assumption that the holonomy along $\gamma$ is sometimes attractive. We fix a pair $\zeta, \zeta^{\prime}$ of numbers from the sequences $\left(\zeta_{n}\right),\left(\zeta_{n}^{\prime}\right)$.

According to Lemma 2.2.1 in [28] and Lemma 2.5.3 in [10] there is a diffeomorphism $g:[-1,1] \rightarrow[-1,1]$ such that

(i) $g$ is the identity outside of $V:=\left(\zeta^{\prime}, \zeta\right)$ and

(ii) $g^{\prime}(z) a(x, 0, z)<a(x, 0, g(z))$ for all $(x, 0, z) \in S^{1} \times\{0\} \times V$. 


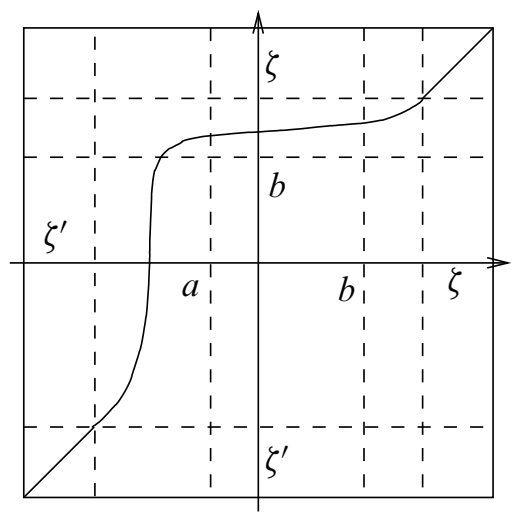

Figure 18

It follows that $g$ converges uniformly to the identity as $\zeta, \zeta^{\prime} \rightarrow 0$, but no claim is made with respect to the $C^{1}$-topology. A typical graph of $g$ is given in Figure 18 (cf [28]). The parameters $a, b$ with $\zeta^{\prime}<a<0<b<\zeta$ are chosen such that $a(x, 0, z) \neq 0$ for $z \in\left[\zeta^{\prime}, a\right] \cup[b, \zeta]$.

In order to obtain the desired confoliation in a $C^{0}$-neighbourhood of $\xi$, one proceeds as follows.

Step 1 Replace $\xi$ on $S^{1} \times[-1 / 2,-1 / 4] \times V$ by the push forward of $\xi$ with the map $G$ which is defined by

$$
G(x, y, z):=(x, y, u(y) g(z)+(1-u(y)) z)
$$

where $u$ is a smooth nonnegative function on $[-1 / 2,-1 / 4]$ such that $u \equiv 0$ near $-1 / 2$ and $u \equiv 1$ near $-1 / 4$. The dashed respectively the solid lines in Figure 19 show the characteristic foliations of $\xi^{\prime}$ on neighbourhoods of $\gamma$ in $\{y=-1 / 4\}$ respectively on $\{y=1 / 2\}$ using dashed respectively solid lines when $\gamma$ has attractive holonomy.

We extend $G$ to $M \backslash\left(S^{1} \times[-1 / 4,1 / 2] \times V\right)$ by the identity. As $\zeta, \zeta^{\prime} \rightarrow 0$ the corresponding diffeomorphism $G$ converges to the identity uniformly but not with respect to the $C^{1}$-topology in general. Therefore $G_{*}(\xi)$ might not be $C^{0}$-close to $\xi$ on $S^{1} \times[-1 / 2,-1 / 4] \times V$. This will be achieved in the third step (at this point we follow the exposition on [28] closely).

Step 2 From $G_{*}(\xi)$ we construct a smooth confoliation $\xi^{\prime \prime}$ on $M$ such that $\partial_{y}$ remains Legendrian: The plane field $\xi^{\prime \prime}$ contains the foliation induced by the second factor of $S^{1} \times[-1 / 4,1 / 2] \times V$ and rotates around these lines. This can be done such that the characteristic foliation on $S^{1} \times\{-1 / 4,1 / 2\} \times V$ coincides with the characteristic 


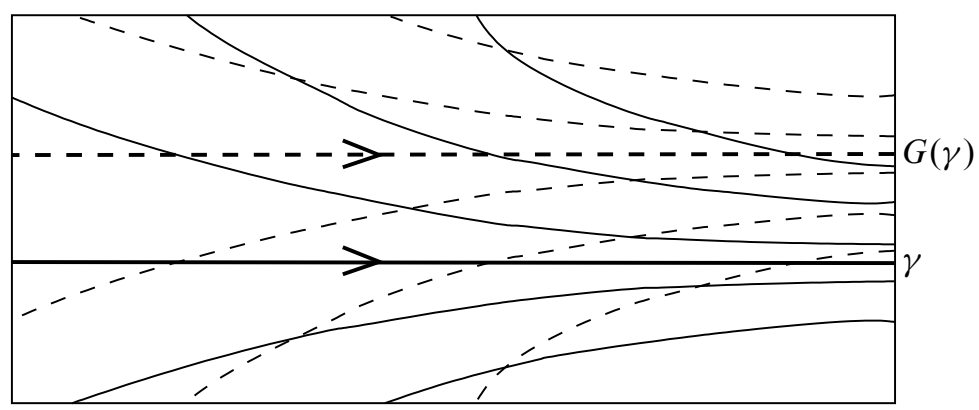

Figure 19

foliation of $G_{*}(\xi)$ on these annuli. This is possible by (ii) using the interpretation of the confoliation condition mentioned in Section 2.3 (cf Figure 19). Note that $\xi^{\prime \prime}$ is a contact structure on the interior of $S^{1} \times[-1 / 4,1 / 2] \times V=: \tilde{V}$.

Step 3 We want to construct a diffeomorphism $\phi$ of $M$ with support in $V$ such that $\phi_{*} \xi^{\prime \prime}$ is $C^{0}$-close to $\xi$. For this one has to choose $V$ more carefully. This is carried out on pages $31-33$ of [28]. The argument can be outlined as follows; cf page 16 in [28]: Assume that $r$ is chosen such that $V \subset[-r / 2, r / 2]$ and $\xi$ is $\varepsilon$-close to the horizontal distribution on $S^{1} \times[-1,1] \times[-r, r]$. As we already mentioned $\xi^{\prime \prime}$ might be very far away from the horizontal distribution. Choose a very small number $\delta>0$ and a diffeomorphism $\varphi:[-r, r] \rightarrow[-r, r]$ such that $\varphi([-r / 2, r / 2]) \subset[-\delta, \delta]$. Then the push forward of the restriction of $\xi^{\prime \prime}$ to $S^{1} \times[-1 / 2,1 / 2] \times[-r, r]$ is $3 \varepsilon$-close to the horizontal distribution. One has to extend $\varphi$ such that this property is preserved.

We will need not only the statement of the lemma, but also the construction outlined in the proof since we need to understand how the modification of $\xi$ near a curve $\gamma$ with sometimes attractive holonomy affects the presence of overtwisted stars on embedded surfaces in $M$. The third step of the above proof is of course irrelevant for this.

Figure 20 shows $F\left(\xi^{\prime \prime}\right)$ near a closed curve of $F\left(\xi^{\prime \prime}\right)$ in an embedded surface $F$ transverse to $\gamma$ after the second step of the proof of Lemma 6.3. The dot in the center of the figure represents $F \cap \gamma$ while the left inner rectangle represents the support of $G$. Finally, $\xi^{\prime \prime}$ is a contact structure in the inner rectangle on the right (this rectangle corresponds to the region $\tilde{V} \cap F$ in the proof of Lemma 6.3). Recall that the characteristic foliation $F(\xi)$ was nearly horizontal in the region shown in Figure 20.

Note that if $\gamma$ has nontrivial infinitesimal (or only attractive) holonomy, then the statement of Lemma 6.3 can be sharpened in the sense that the lemma remains true for $C^{\infty}$-neighbourhoods of $\xi$ because the function $g:[-1,1] \rightarrow[-1,1]$ can be chosen 


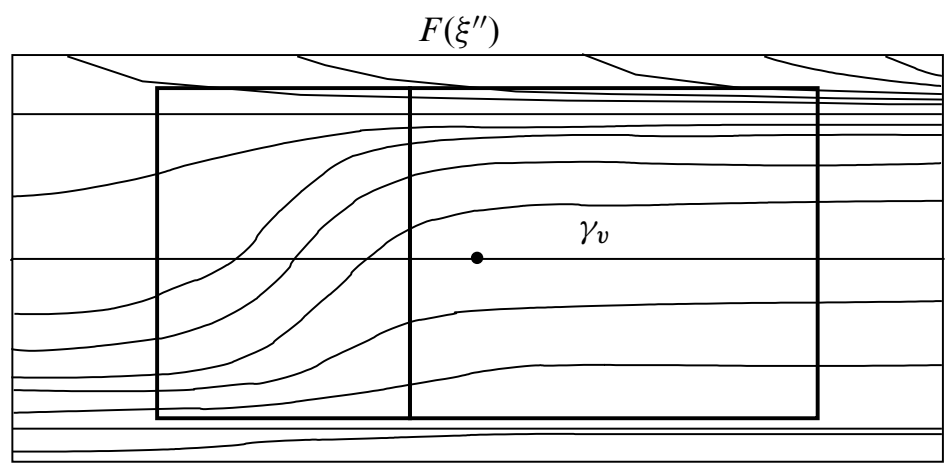

Figure 20

$C^{\infty}$-close to the identity. In the following we will consider only $C^{0}$-approximations. This allows us to choose the approximation of $\xi$ more freely. In particular, we can preserve qualitative features of the characteristic foliation on surfaces transverse to $\gamma$.

Lemma 6.4 Let $\xi$ be a $C^{k}$-confoliation, $k \geq 1$, and $\gamma$ a simple Legendrian segment such that both endpoints of $\gamma$ lie in the contact region and $\gamma$ intersects $F$ transversely and at most once.

Then every $C^{k}$-neighbourhood of $\xi$ contains a confoliation $\xi^{\prime}$ such that $\xi^{\prime}=\xi$ outside a neighbourhood of $\gamma$ and $\xi^{\prime}$ is a contact structure on a neighbourhood of $\gamma$. Moreover, $F(\xi)=F\left(\xi^{\prime}\right)$.

Proof The case $\gamma \cap F=\varnothing$ corresponds to Lemma 2.8.2 in [10], the case $\gamma \cap F=\{p\}$ is very similar and only this case uses the assumption that both endpoints of $\sigma$ lie in $H(\xi)$.

The following lemma is standard in the setting of foliations: One can thicken a closed leaf to obtain a smooth foliation which is close to the original one and contains a family of closed leaves. The new difficulty in the context of confoliations is the fact that now compact leaves of $\xi$ may have boundary.

Lemma 6.5 Let $\xi$ be a confoliation on $M, L \subset M$ a compact embedded surface tangent to $\xi$ and $F \subset M$ a closed oriented surface which is generically embedded and does not intersect $\partial L$. We require that each connected component of $\partial L$ can be

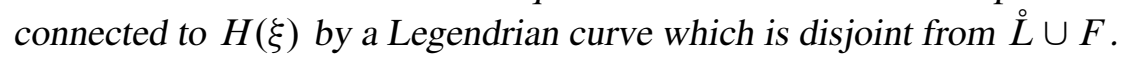

Then there is a smooth confoliation $\xi^{\prime}$ which is $C^{0}$-close to $\xi$ such that $F\left(\xi^{\prime}\right)$ is homeomorphic to the singular foliation obtained from $F(\xi)$ by thickening the closed leaves of cycles of $F(\xi)$ which are also contained in $L$. 
Proof Let $I=[-1,1]$ and $J=[-1,0]$. We fix a tubular neighbourhood $U \simeq L \times I$ of $L=L \times\{0\}$.

For each boundary component $B_{i}$ of $L$ we choose $U_{i} \simeq S^{1} \times J \times I \subset M$ in the complement of $\stackrel{\circ}{L} \cup F$. We assume that the third factor of $U_{i}$ is transverse to $\xi$ while the foliation $\mathcal{J}$ whose fibers correspond to the second factor is Legendrian and that $S^{1} \times\{(0,0)\}=B_{0, i}$ and $S^{1} \times\{(-1,0)\}=B_{-1, i}$ intersect $H(\xi)$. Let $A_{j, i}=S^{1} \times\{j\} \times I \subset \partial U_{i}$ for $j \in\{-1,0\}$.

Without loss of generality we may assume that $B_{-1, i}$ is completely contained in the contact region and transverse to $\xi$. (Otherwise apply Lemma 6.4 to segments of $B_{-1, i}$ and replace $U_{i}$ with a new set $U_{i}^{\prime}$ with the desired property.)

We will now construct a confoliation $\xi^{\prime}$ on $U \cup \bigcup_{i} U_{i}$ which coincides with $\xi$ near $\partial U$ and has the desired properties.

The restriction of $\xi^{\prime}$ to $U$ is defined in two steps. First, we flatten $\xi$ in a neighbourhood $U \simeq L \times I$ using the push forward of $\xi$ by $g \circ g$ where $g$ is a smooth homeomorphism of $I$ which is $C^{\infty}$-tangent to the zero map and coincides with the identity outside a neighbourhood of 0 .

We push forward $\xi$ on $L \times[0,1]$ respectively $L \times[-1,0]$ using orientation preserving homeomorphism $[0,1] \rightarrow[\varepsilon, 1]$ respectively $[-1,0] \rightarrow[-1,-\varepsilon]$ which is smooth away from 0 . The confoliation on $(L \times[-1,-\varepsilon]) \cup(L \times[-\varepsilon, \varepsilon]) \cup(L \times[\varepsilon, 1]) \simeq U$ (with $\varepsilon>0$ ), which is the product foliation on $L \times[-\varepsilon, \varepsilon]$, is smooth and contains a family of compact leaves. We can choose the diffeomorphisms appearing in the construction such that $\left.\xi\right|_{U}$ is as close to $\left.\xi^{\prime}\right|_{U}$ in the $C^{0}$-topology as we want. As in the third step of the proof of Lemma 6.3 one can ensure that the resulting distribution is $C^{0}$-close to the original confoliation.

We can choose $\left.\xi^{\prime}\right|_{U}$ such that $A_{0, i}(\xi)$ and $A_{0, i}\left(\xi^{\prime}\right)$ coincide outside of the region where the slope of $A_{0, i}(\xi)$ is very small compared to the slope of $A_{-1, i}(\xi)$. By construction the slope of $A_{0, i}\left(\xi^{\prime}\right)$ is much smaller than the slope of $A_{-1, i}(\xi)=A_{-1, i}(\xi)$. As in the second step in the proof of Lemma 6.3 (or Proposition 2.5.1 of [10]) one can extend $\xi^{\prime}$ to a smooth confoliation on $M$ such that $\xi^{\prime}$ is close to $\xi$ (the foliation $\mathcal{J}$ corresponds to the $y$-coordinate in [10]). The claim about $F\left(\xi^{\prime}\right)$ follows immediately from the construction.

Remark 6.6 After a trivially foliated bundle $L \times[-\varepsilon, \varepsilon]$ is added to the confoliation, it is possible to replace the trivially foliated piece by a foliation on $L \times[-\varepsilon, \varepsilon]$ such that the boundary leaves $L \times\{ \pm \varepsilon\}$ have sometimes attractive holonomy on side lying in $L \times[-\varepsilon, \varepsilon]$. The following statements follow from the construction explained in 
[10, page 39]. (This construction carries over to surfaces with boundary after the surface is doubled.)

When the Euler characteristic of $L$ is negative, then one can replace the product foliation on $L \times[-\varepsilon, \varepsilon]$ by a foliation such that the holonomy along every homotopically non trivial curve in $L \times\{\varepsilon\}$ or $L \times\{-\varepsilon\}$ is sometimes attractive on the side in $L \times[-\varepsilon, \varepsilon]$.

If the Euler characteristic of the compact surface with boundary $L$ is not negative, then $L$ is diffeomorphic to $S^{2}, D^{2}, T^{2}$ or $S^{1} \times I$. The case $S^{2}$ will not occur unless the confoliation in question is actually a product foliation by spheres. But these are excluded. If $L \simeq S^{1} \times I$, then the suspension of a suitable diffeomorphism yields the same result as in the case of $\chi(L)<0$ (without doubling the surface). The case $L \simeq D^{2}$ will be excluded by the last requirement of Definition 6.1 in the application we have in mind. Finally, the case $L \simeq T^{2}$ is exceptional because of Kopell's lemma (cf the footnote on page 39 of [10]). But if $L=T^{2}$, then it is easy to arrange that the holonomy is attractive along a given homotopically nontrivial curve.

This modification changes the characteristic foliation on $F$, but only an open set which was foliated by closed leaves and cycles before the perturbation. In particular, overtwisted stars are not affected.

The following proposition from [10] adapts a famous result of Sacksteder [30] to laminations so that it can be applied to the fully foliated part of confoliations.

Proposition 6.7 [10, Proposition 1.2.13] Let $(M, \xi)$ be a $C^{k}$-confoliation, $k \geq 2$, which is not a foliation with dense leaves. All minimal sets of the fully foliated part of $\xi$ are either closed leaves or exceptional minimal sets. Each exceptional minimal set contains a simple closed curve along which $\xi$ has nontrivial infinitesimal holonomy.

In particular, exceptional minimal sets are isolated and there are only finitely many of them.

We denote the finite set consisting of exceptional minimal sets of the fully foliated part of $\xi$ by $\mathcal{E}(\xi)$.

In the following $F$ will be an embedded surface containing an overtwisted star $(Q, V, \alpha)$. We write $\Omega_{Q}$ for $\bigcup_{v \in V} \gamma_{v}$. We will be mostly concerned with limit sets $\gamma_{v}, v \in V$ which are contained in compact leaves (with or without boundary) or in exceptional minimal sets of the fully foliated part of $M$. By Lemma 6.5 and the remark following it, we may assume that if $\gamma_{v}, v \in V$ is a cycle lying in a compact leaf, then $\gamma_{v}$ contains no hyperbolic singularities. 
Lemma 6.8 Let $\xi$ be a confoliation and $F$ an embedded connected surface containing an overtwisted star $(Q, V, \alpha)$ and $v \in V$. The space of plane fields on $M$ carries the $C^{0}$-topology.

(a) If $\gamma_{v}$ is contained in a closed leaf of $\xi$, then in every neighbourhood of $\xi$ there is a confoliation $\xi^{\prime}$ such that $F\left(\xi^{\prime}\right)$ contains an overtwisted star $\left(Q^{\prime}, V^{\prime}, \alpha^{\prime}\right)$ which is naturally identified with $(Q, V, \alpha)$ and $\gamma_{v}^{\prime},\left(v \in V^{\prime} \simeq V\right)$ passes through the contact region of $\xi^{\prime}$.

(b) Assume that $\gamma$ is contained in an exceptional minimal set, $\gamma$ has attractive linear holonomy, and $\gamma$ is transverse to $F$. Then every $C^{0}$-neighbourhood of $\xi$ contains a confoliation $\xi^{\prime}$ such that $F\left(\xi^{\prime}\right)$ contains an overtwisted star which can be naturally identified with $(Q, V, \alpha)$ and $\left|\mathcal{E}\left(\xi^{\prime}\right)\right|<|\mathcal{E}(\xi)|$.

Proof First, we prove (a). Let $L$ be the closed leaf containing $\gamma_{v}$. Since $\gamma_{v}$ is the $\omega$-limit set of leaves in $F(\xi)$ it has attractive holonomy on one side and $F \cap L$ consists of a family of cycles. In particular, $L \cap \alpha(Q)=\varnothing$ because an overtwisted star with virtual vertices does not contain closed cycles of the characteristic foliation.

We use Lemma 6.5 and Remark 6.6 to ensure that $\gamma_{v}$ has sometimes attractive holonomy on both sides. Unfortunately this property is not stable under arbitrary isotopies of $\gamma_{v}$ in general. But by Lemma 2.1 there is an annulus $A \simeq \gamma_{v} \times[0,1]$ such that $\gamma_{v}=\gamma_{v} \times\{0\}=F \cap A$ and all curves in $A$ have attracting holonomy on the side where $\alpha(Q)$ approaches $\gamma_{v}$ while isotopies do not change the nature of the holonomy on the other side of $L$ since by construction the confoliation is actually a foliation there.

Therefore there is a small isotopy of $F$ which maps $(Q, V, \alpha)$ to an overtwisted star $\left(Q^{\prime}, V^{\prime}, \alpha^{\prime}\right)$ on the isotoped surface $F^{\prime}$ such that $\gamma_{v}$ is mapped to $\gamma_{v} \times\{\varepsilon\}$ where $0<\varepsilon<1 / 2$. Then we can apply Lemma 6.3 to $\gamma_{v} \times\{0\}$ and $\gamma_{v} \times\{2 \varepsilon\}$.

Now there is a Legendrian arc intersecting $F^{\prime}$ exactly once in a point of $\gamma_{v}$ and both endpoints of this arc lie in the contact region. Hence this arc satisfies the assumptions of Lemma 6.4. This yields a confoliation $\xi^{\prime}$ with the desired properties such that $F^{\prime}(\xi)=F^{\prime}\left(\xi^{\prime}\right)$. This finishes the proof of (a).

Now we prove (b). We shall use notation from the proof of Lemma 6.3. In the proof we will use the freedom in the choice of the function $g$ in the proof of Lemma 6.3. For this we need the fact that $\gamma$ has nontrivial infinitesimal holonomy, since then there are only very few restrictions on $g$ in the proof of Lemma 6.3; cf also Lemma 2.5.2 in [10].

Fix a neighbourhood $U \simeq S_{x}^{1} \times[-1,1]_{y} \times[-1,1]_{z}$ such that $\gamma=S^{1} \times\{(0,0)\}$ and the coordinates $x, y, z$ have all properties used in the proof of Lemma 6.3. In particular, 
the foliation by the second factor is Legendrian and coincides with $F(\xi)$ on $F \cap U$ while the third factor is positively transverse to $\xi$. We require that $U$ intersects $F$ only in neighbourhoods of points in $\gamma \cap \Omega_{Q}=: X$.

Let us make an orientation assumption in order to simplify the presentation: We assume that the orientation of the Legendrian foliation on $S^{1} \times[-1,1] \times[-1,1]$ given by the second factor coincides with the orientation of $F(\xi)$ near points of $\gamma \cap \gamma_{v}, v \in V$, ie in Figure 20 the foliation is oriented from left to right. When this assumption is not satisfied for some $y \in \gamma \cap \Omega_{Q}$, then one has to interchange the roles of $\hat{\tau}_{-}(y)$ and $\widehat{\tau}_{+}(y)$ in some of the following arguments.

By transversality $\gamma$ intersects $F$ in a finite number of points. Since $\gamma$ is contained in the fully foliated part of $\xi, \gamma$ cannot intersect $\alpha(Q)$ since every point of $\alpha(Q)$ is connected to $H(\xi)$ by a Legendrian arc. We can ignore the points in $F \cap \gamma$ which do not belong to $\overline{\alpha(Q)}$ if we deform $\xi$ on a neighbourhood of $\gamma$ which is small enough.

Because $F$ is smoothly embedded and $\xi$ is smooth, $F(\xi)$ is also smooth. As we have already mentioned in Section 3.2 the $\omega$-limit set $\gamma_{v}$ with $v \in V$ is either a quasi-minimal set or we may assume that $\gamma_{v}$ is a closed leaf of $F(\xi)$. We distinguish the following cases.

(i) $\gamma_{v}$ is quasi-minimal. Since there are interior points of $\alpha(Q)$ arbitrarily close to $\gamma_{v}$, there is no segment $\tau$ transverse to $F(\xi)$ such that $\tau \cap \gamma_{v}$ is dense in $\tau$. Then $\gamma_{v} \cap \tau$ is a Cantor set (cf [18]). Recall from Section 2.2 that the intersection between two different quasi-minimal sets cannot contain a recurrent orbit and the number of quasi-minimal sets of $F(\xi)$ is bounded by the genus of $F$.

(ii) $\gamma_{v}$ is a closed leaf of $F(\xi)$ whose holonomy is attractive on the side from which $\alpha(Q)$ accumulates on $\gamma_{v}$ while it is repulsive on the other side and $\alpha(Q)$ spirals onto $\gamma_{v}$ on the attractive side. In this case, $\alpha(Q)$ cannot enter a one-sided neighbourhood of $\gamma_{v}$ on the side where the holonomy is repulsive.

(iii) $\gamma_{v}$ is a closed leaf of $F(\xi)$ whose holonomy is attractive on one side and either there is a sequence of closed leaves of $F(\xi)$ on the other side of $\gamma_{v}$ which converge to $\gamma_{v}$ or $\gamma_{v}$ has attractive holonomy on both sides.

If $\gamma_{v}$ belongs to class (iii) and $U$ is small enough (ie contained in the interior of an annulus each of whose boundary is tangent to $F(\xi)$ or transverse to $F(\xi)$ such that $F(\xi)$ points into the annulus along the boundary), then any modification of $F(\xi)$ with support in $U \cap F$ will result in a singular foliation on $F$ such that all leaves of the characteristic foliation which enter a neighbourhood of $\gamma_{v}$ containing $U$ will remain in $U$ forever even after the modification. When no singularities are created during the modification, then the modification replaces $(Q, V, \alpha)$ by an overtwisted star 
$\left(Q^{\prime}, V^{\prime}, \alpha^{\prime}\right)$ such that $|V|=\left|V^{\prime}\right|$. In this case $\gamma_{v} \neq \gamma_{v}^{\prime}$ but $\gamma_{v}^{\prime}$ is a closed leaf of $F\left(\xi^{\prime}\right)$ which passes through $H\left(\xi^{\prime}\right)$ (by the proof of Lemma 6.3. We keep this case separated from the others although all three of them may occur in one single perturbation of $\xi$.

The following argument is complicated by a difficulty in case (ii). If $\alpha(Q)$ accumulates on $\gamma_{v}$ and the holonomy of $\gamma_{v}$ is repulsive on the side where points of $\gamma$ are pushed to by the diffeomorphism $G$ appearing in the proof of Lemma 6.3, then it is impossible to say something about the new $\omega$-limit set of leaves in $\alpha(Q)$ which accumulated on $\gamma_{v}$ unless $G$ is chosen carefully: It is possible that leaves which accumulated on $\gamma_{v}$ accumulate on $\gamma_{v^{\prime}}$ when the characteristic foliation is modified near $\gamma_{v}$. However it is possible that $\gamma_{v^{\prime}}$ is also changed when $\xi$ is replaced by $\xi^{\prime}$. Therefore one has to treat all $v \in V$ such that $\gamma_{v}$ belongs to (i),(ii) simultaneously.

For nonempty open intervals $\tau_{-} \subset[-1,0)$ and $\tau_{+} \subset(0,1]$ we write $\hat{\tau}_{ \pm}(y):=\{y\} \times$ $[-1,1] \times \overline{\tau_{ \pm}}$for $y \in \gamma$. We will fix $\tau_{ \pm}$in the following.

We require that $\tau_{+}$is chosen such that the $\omega$-limit of a leaf intersecting $\hat{\tau}_{+}(y)$ is never a hyperbolic singularity for all $y \in X$. Because

- there are only finitely many hyperbolic singularities on $F$,

- $\alpha(Q)$ intersects every interval transverse to $\gamma_{v}$ in an open set (note that there are singular foliations on surfaces with dense quasi-minimal sets; in particular stable leaves of hyperbolic singularities in such quasi-minimal sets may be dense in the surface) and

- $\alpha(\partial Q)$ is disjoint from those $\gamma_{v}, v \in V$ which intersect $\gamma$ even if $\gamma_{v}$ is quasiminimal (this is true because every point of $\alpha(Q)$ is connected to $H(\xi)$ by a Legendrian curve while $\gamma$ is part of the fully foliated set),

this condition can be satisfied. Next we impose additional restrictions on $\tau_{-}$:

We choose $\tau_{-}$such that no point in $\hat{\tau}_{+}(x)$ is connected to $\hat{\tau}_{-}(y)$ for $x, y \in X$ by a leaf of $F(\xi)$ which is disjoint from $\{(y, 0)\} \times\left[\sup \left(\tau_{-}\right)\right.$, sup $\left.\left(\tau_{+}\right)\right]$. In other words, we require that leaves of $F(\xi)$ which come from $\hat{\tau}_{+}(x)$ do not intersect $\hat{\tau}_{-}(y)$ when they meet the piece of $\{(y,-1)\} \times[-1,1] \subset(U \cap F)$ which lies between the lower endpoint of $\hat{\tau}_{-}(y)$ and the upper endpoint of $\hat{\tau}_{+}(y)$ for the first time. In order to satisfy this condition it might be necessary to shorten $\tau_{+}$.

There is a choice for $\tau_{+}, \tau_{-}$which satisfies these requirements for $x, y \in X$ whenever the limit set $\gamma_{v}$ which corresponds to $y$ is not the $\omega$-limit set of leaves intersecting $\hat{\tau}_{+}(x)$.

If $y$ is contained in a closed leaf of $F(\xi)$, then one can also satisfy the requirement for $x, y \in X$ provided that $\tau_{+}$is so short that the translates of $\hat{\tau}_{+}(x)$ along leaves 
of $F(\xi)$ do not cover the segment $\left.\hat{\tau}_{-}(y)\right)$. We shorten $\tau_{+}$whenever this is necessary. Finally, when $y$ is part of a quasi-minimal set and the leaves of $F(\xi)$ which intersect $\hat{\tau}_{+}(x)$ accumulate on this quasi-minimal set the above requirement can be satisfied by shortening $\tau_{ \pm}$again. Now one can construct $\tau_{-}$in a finite number of steps and shortening $\tau_{ \pm}$at each step.

Let $t_{-} \in \tau_{-}$. We fix the diffeomorphism $g:[-1,1] \rightarrow[-1,1]$ in the proof of Lemma 6.3 such that $g$ maps the entire interval $\left(t_{-}, \sup \left(\tau_{+}\right)\right)$into $\tau_{+}$and the support of $g$ is contained in $\left(\inf \left(\tau_{-}\right), \sup \left(\tau_{+}\right)\right)$. The role of the parameters $\zeta, \zeta^{\prime}$ from the proof of Lemma 6.3 is now played by $\sup \left(\tau_{+}\right), \inf \left(\tau_{-}\right)$.

If $\xi$ is modified by the procedure described in the proof of Lemma 6.3 using the diffeomorphism $g$ chosen above, then one obtains a confoliation $\xi^{\prime}$ such that all leaves of $F\left(\xi^{\prime}\right)$ starting at the elliptic singularity in the center of the original overtwisted whose $\omega$-limit set was $\gamma_{v}$ such that $\gamma_{v} \cap \gamma \neq \varnothing$ never meet a hyperbolic singularity of $F\left(\xi^{\prime}\right)$.

Since all elliptic singularities on the boundary of the basin of the elliptic singularity in $\alpha(Q)$ are automatically negative and all hyperbolic singularities on the boundary of the basin where already present in $\alpha(\partial Q)$ there is an overtwisted star $\left(Q^{\prime}, V^{\prime}, \alpha^{\prime}\right)$ and $V^{\prime}$ can be viewed as a subset of $V$ by construction. Moreover, $\left|\mathcal{E}\left(\xi^{\prime}\right)\right|<|\mathcal{E}(\xi)|$.

Now we can finally show that there are no overtwisted stars when $\xi$ is symplectically fillable.

Theorem 6.9 Let $(M, \xi)$ be a $C^{k}$-confoliation, $k \geq 2$, which is symplectically fillable. Then $\xi$ is s-tight.

Proof Let $(X, \omega)$ be a symplectic filling of $\xi$. According to Theorem $1.4 x i$ is tight (below we will outline a proof of Theorem 1.4). Assume that $F$ is an embedded surface containing an overtwisted star $(Q, V, \alpha)$. It is sufficient to treat only the case of closed surfaces when the elliptic singularity in the interior of $\alpha(Q)$ is positive.

In the first part of the proof we show how to reduce the number of virtual vertices. Because overtwisted stars are not required to be injective as Legendrian polygons, we show in a second step how to obtain an embedded disc $D$ such that $\partial D$ is Legendrian and $\left.T D\right|_{\partial D}$ is transverse to $\left.\xi\right|_{\partial D}$ violating Definition 1.3 starting from an overtwisted star $(Q, \varnothing, \alpha)$. The confoliation is modified several times but all confoliations appearing in the proof will be $C^{0}$-close to $\xi$. In particular, they are symplectically fillable. Therefore the assumption that $(M, \xi)$ admits an overtwisted star contradicts Theorem 1.4. 
Notice that in the presence of an overtwisted star $\xi$ cannot be a foliation everywhere. Therefore $M$ is not a minimal set of the fully foliated part of $\xi$ and $\xi$ is not a foliation without holonomy.

Step 1 If $V \neq \varnothing$, then $\xi$ can be approximated by a confoliation which admits an overtwisted star with less virtual vertices than $(Q, V, \alpha)$.

We fix $v_{0} \in V$. If $\gamma_{0}:=\gamma_{v_{0}}$ intersects $H(\xi)$, then an application of Lemma 3.6 yields a surface carrying an overtwisted star with less virtual vertices. Now assume $\gamma_{0} \cap H(\xi)=\varnothing$.

Let $L$ be the maximal connected open immersed hypersurface of $M$ which is tangent to $\xi$ and contains $\gamma_{0}$. If $L=\varnothing$, then there is a Legendrian segment $\sigma$ satisfying the hypothesis of Lemma 6.4. After applying this lemma, $\gamma_{v}$ intersects the contact region of the modified confoliation and we are done.

Now assume $L \neq \varnothing$ and let $L^{\infty}$ be the space of ends of $L$. We say that an end $e \in L^{\infty}$ lies in $H(\xi)$ if for every compact set $K \subset L$ there is a Legendrian curve from $H(\xi)$ to the connected component of $L \backslash K$ corresponding to $e$ and the curve avoids $K$.

Step 1a If $L^{\infty} \neq \varnothing$, then we approximate $\xi$ such that all ends of $L$ lies in the contact region of the modified confoliation.

The set of ends in $H(\xi)$ is open in $L^{\infty}$, therefore its complement $L_{\text {fol }}^{\infty}$ is compact. To each $e \in L_{\text {fol }}^{\infty}$ we associate a minimal set $\mathcal{M}(e) \subset \lim _{e} L$ of the fully foliated part of $\xi$ (this is explained in [5, page 115]). Recall that $M$ cannot be a minimal set of the fully foliated part of $\xi$. Hence according to [19, page 19], all minimal sets are either closed leaves or exceptional minimal sets. Note that $L$ may be contained in $\mathcal{M}(e)$.

If $\mathcal{M}(e)$ is a closed leaf of $\xi$ whose holonomy along a curve $\gamma$ transverse to $F$ is sometimes attractive, then we can apply Lemma 6.8 (a) to $\gamma_{v}$ if there is $v \in V$ with $\gamma_{v} \subset \mathcal{M}(e)$. If $L$ contains no limit set of $\alpha(Q)$, then the procedure from the proof of Lemma 6.3 can be applied directly to any curve $\gamma \subset \mathcal{M}(e)$ with sometimes attractive holonomy. We can ensure the existence of such a curve by Lemma 6.5 and Remark 6.6.

If $\mathcal{M}(e)$ is an exceptional minimal set, then according to Proposition 6.7 there is a simple closed curve $\gamma$ in a leaf $L_{\gamma} \subset \mathcal{M}(e)$ with nontrivial infinitesimal holonomy. Every curve in $L_{\gamma}$ which is isotopic to $\gamma$ through Legendrian curves has the same property by Lemma 1.3.17 in [10]. In particular, we may assume that $\gamma$ is transverse to $F$. Using Lemma 6.8 (b) we approximate $\xi$ by a confoliation $\xi^{\prime}$ such that $L_{\gamma}$ meets $H\left(\xi^{\prime}\right)$. 
If $\mathcal{M}(e)$ was an exceptional minimal set, this process might have changed the overtwisted star in the sense that type of the $\omega$-limit sets of virtual vertices may have changed. But recall that by the proof of Lemma 6.8 we can view $V^{\prime}$ as a subset of $V$. We use $\gamma_{v}^{\prime}$ to denote the $\omega$-limit set of leaves which start at the elliptic singularity in the center of the overtwisted star and accumulated on $\gamma_{v}, v \in V$ before the modification.

We iterate the procedure from the very beginning with $v_{0} \in V^{\prime}$ and with an integral surface of $\xi^{\prime}$ containing $\gamma_{0}^{\prime}$. Since $\mathcal{E}(\xi)$ is finite and $\left|\mathcal{E}\left(\xi^{\prime}\right)\right|<|\mathcal{E}(\xi)|$ this phenomenon can occur only finitely many times.

In later applications of the above construction $\gamma_{0}^{\prime}=\gamma_{0}$ and the maximal integral surface of $\xi^{\prime}$ containing $\gamma_{0}^{\prime}$ is contained in the maximal integral surface of $\xi$ containing $\gamma_{0}$. Because the inclusion induces a continuous mapping between the spaces of ends and by the compactness of $L_{\mathrm{fol}}^{\infty}$ we are done after finitely many steps. We continue to write $F$ for the embedded surface, $\xi$ for the confoliation, $(Q, V, \alpha)$ for the overtwisted star, etc.

Step 1b We isotope $F$ such that all quasi-minimal sets of the characteristic foliation on the resulting surface pass through the contact region.

As we have already noted in the proof of Lemma $6.8, F(\xi)$ has only finitely many quasi-minimal sets (this number is bounded by the genus of $F$ ). Let $\gamma_{w}, w \in V$ be a quasi-minimal set of $F(\xi)$ which is disjoint from $H(\xi)$.

According to Theorem 2.3.3 in [26] there is an uncountable number of leaves of $F(\xi)$ which are recurrent (in both directions) and dense in $\gamma_{w}$ while $(Q, V, \alpha)$ has only a finite number of pseudovertices and $|V|<\infty$. Therefore there is $p_{w} \in \gamma_{w}$ which can be connected to $H(\xi)$ by a Legendrian arc $\sigma$ transverse to $F$ such that $\sigma$ does not meet $\alpha(\partial Q)$ and $\sigma$ never intersects cyclic components of $\Omega_{Q}$. At this point we use that every end of the union of integral hypersurfaces containing $\gamma_{w}$ lies in $H(\xi)$. If $\sigma$ intersects $\Omega_{Q}$ in some other quasi-minimal set $\gamma_{w^{\prime}}, w^{\prime} \in V$ before it meets $H(\xi)$, then we replace $\gamma_{w}$ by $\gamma_{w^{\prime}}$. Thus we may assume that $\sigma$ meets $F$ in $p_{w}$ and nowhere else.

By Lemma 2.8.2 in [10] there is a confoliation $\xi^{\prime} C^{k}$-close to $\xi$ such that $F\left(\xi^{\prime}\right)=F(\xi)$, $\sigma$ is tangent to $\xi$ and $\xi^{\prime}$ and a neighbourhood of $p_{w}$ in $F$ lies in $\overline{H\left(\xi^{\prime}\right)}$. We will denote $\xi^{\prime}$ again by $\xi$.

Choose a neighbourhood $U \simeq \sigma \times[-1,1] \times[-1,1]$ of $\sigma$ such that $\sigma=\sigma \times\{(0,0)\}$ and $\left(\left\{p_{w}\right\} \times[-1,1] \times[-1,1]\right) \subset F$. Moreover, we require that the foliation by the first factor is Legendrian while the foliation corresponding to the second factor is transverse to $\xi$ and $\stackrel{\circ}{U} \subset H(\xi)$. Finally we assume that the foliation which corresponds to the second factor is Legendrian when it is restricted to $F$. 
Now we apply an isotopy to $F$ whose effect on the characteristic foliation on $F$ is the same as the effect of the map $G$ appearing in the proof of Lemma 6.3. We explain this under the following orientation assumptions (the other cases can be treated in the same way): The orientation of $F(\xi)$ coincides with the second factor of $U \simeq \sigma \times[-1,1] \times[-1,1]$ and the coorientation of $F$ points away from $U$.

In Figure 20 the left respectively right edge of the rectangle corresponds to $\left\{\left(p_{w},-1\right)\right\} \times$ $[-1,1]$ respectively $\left\{\left(p_{w}, 1\right)\right\} \times[-1,1]$, the foliation is oriented from left to right, the coorientation of $\xi$ points upwards and the coorientation of $F$ points towards the reader.

Choose $-1<x<0<y<1$ such that the points $\left(p_{w},-1, x\right),\left(p_{w}, 1, y\right) \in F$

(i) do not lie on a stable or unstable leaf of a hyperbolic singularity and they are not connected by a leaf of $F(\xi)$ and

(ii) can be connected by a smooth Legendrian arc $\lambda$ in $U$ whose projection to $\sigma \times[-1,1]$ is embedded and $\lambda$ is $C^{\infty}$ tangent to $F$. Moreover, we assume that the projection of $\lambda$ to $\sigma \times[-1,1]$ is transverse to the first factor.

The curve $\lambda$ and the points $x, y$ exist because of the orientation assumptions and Lemma 2.1. Now fix $x^{\prime}, y^{\prime}$ close to $x, y$ such that $x<x^{\prime}<0<y^{\prime}<y$.

Using a flow along the first factor of $U$ we can move $\left\{p_{w}\right\} \times[-1,1]$ to a curve which is close to the projection of $\lambda$ to $\sigma \times[-1,1]$. When we apply this flow to $F$, the surface is pulled into $U$ and we obtain a surface $F^{\prime}$ isotopic to $F$ which coincides with $F$ outside of $\left\{p_{w}\right\} \times(-1,1) \times(x, y)$.

By the assumptions on $\lambda$ we can choose $F^{\prime}$ such that the holonomy of $F^{\prime}(\xi)$ maps the transverse segment $\left\{\left(p_{w},-1\right)\right\} \times\left(x^{\prime}, y\right)$ onto $\left\{\left(p_{w}, 1\right)\right\} \times\left(y^{\prime}, y\right)$ such that no leaf of intersecting $\left\{\left(p_{w}, 1\right)\right\} \times\left(y^{\prime}, y\right)$ is part of a stable or unstable leaf of $F(\xi)$. Moreover, we may assume that leaves which start at points of $\left\{\left(p_{w}, 1\right)\right\} \times\left(y^{\prime}, y\right)$ meet the segment $\left\{\left(p_{w},-1\right)\right\} \times\left[x^{\prime}, y\right]$ before the enter the region where $F^{\prime} \neq F$ for the first time. The new $\omega$-limit set is now a closed leaf of $F^{\prime}(\xi)$ which passes through $\left\{\left(p_{w}, 1\right)\right\} \times\left(y^{\prime}, y\right)$.

This modification may have created quasi-minimal sets on $F^{\prime}$ which were not present in $F(\xi)$. But if this happens, then the new quasi-minimal sets intersect the contact region by construction. After a finite number of steps (bounded by the genus of $F$ ) we have isotoped $F$ such that all quasi-minimal sets of the characteristic foliation on the resulting surface pass through the contact region. Now we apply Lemma 3.6. We obtain a surface $F^{\prime \prime}$ containing an overtwisted star $\left(Q^{\prime \prime}, V^{\prime \prime}, \alpha^{\prime \prime}\right)$ such that there is a natural inclusion $V^{\prime \prime} \subset V$ and all $\gamma_{v}, v \in V^{\prime \prime}$ are cycles of $F^{\prime \prime}(\xi)$. We will denote $F^{\prime \prime}$ by $F, Q^{\prime \prime}$ by $Q$, etc. 
Step 1c In this step we reduce the number of virtual vertices.

Let $\gamma_{0}$ be the limit set which corresponds to the virtual vertex $v_{0} \in V$ of an overtwisted star $(Q, V, \alpha)$. We assume that $\gamma_{v}$ is a cycle for all $v \in V$ and all ends of the maximal integral surface $L_{0}$ containing $\gamma_{0}$ lie in the contact region.

Choose a submanifold $L_{0}^{\prime} \subset L_{0}$ of dimension 2 such that $L_{0}^{\prime}$ contains all closed components of $\Omega_{Q} \cap L_{0}$. Since each end of $L_{0}$ lies in $H(\xi)$ we can choose $L_{0}^{\prime}$ so that each boundary component is connected to $H(\xi)$ by a Legendrian curve which does not intersect the interior of $L_{0}^{\prime}$. After a $C^{\infty}$-small perturbation (we use again Lemma 2.8.1 from [10]) of $\xi$ we may assume that the boundary of $L_{0}^{\prime}$ is contained in the contact region of the resulting confoliation $\xi^{\prime}$. This perturbation might affect the characteristic foliation on $F$, but since the modification of the confoliation does not affect $\Omega_{Q}$ and all components of $\Omega_{Q}$ are cycles of $F(\xi)$ which are also present in $F\left(\xi^{\prime}\right)$, there still is an overtwisted star $\left(Q^{\prime}, V^{\prime} \alpha^{\prime}\right)$ on $F$ together with a natural inclusion $V^{\prime} \hookrightarrow V$.

Now we can apply Lemma 6.5 and Remark 6.6. From Lemma 6.8 (a) we obtain a confoliation $\xi^{\prime \prime}$ which is $C^{0}$-close to $\xi^{\prime}$ such that $F\left(\xi^{\prime \prime}\right)$ contains an overtwisted star $\left(Q^{\prime \prime}, V^{\prime \prime}, \alpha^{\prime \prime}\right)$ with $V^{\prime \prime} \subset V^{\prime}$ and all $\omega$-limit sets $\gamma_{w}^{\prime \prime}, w \in V^{\prime \prime}$ which were contained in $L_{0}$ now intersect the contact region of $\xi^{\prime \prime}$. Using Lemma 3.6 we reduce the number of virtual vertices.

Step 2 We show that we can assume that the map $\alpha$ associated to the overtwisted star $(Q, \varnothing, \alpha)$ in $F$ is injective.

Assume that the Legendrian polygon $(Q, \varnothing, \alpha)$ is not injective. We first treat the case, that there is a nontrivial cycle $\gamma_{w_{1}} \ldots w_{l}$. In order to simplify the notation in the following we assume that $\gamma_{w_{1}} \ldots w_{l}$ contains no corners except $\alpha\left(w_{i}\right), i=1, \ldots l$. Let $\eta$ be the stable leaf of $\alpha\left(w_{1}\right)$ which is contained in $\gamma w_{1} \ldots w_{l}$ of $\alpha\left(w_{1}\right)$, by our assumption $\eta$ is also an unstable leaf of $\alpha\left(w_{2}\right)$. We choose a Legendrian curve $\sigma_{\eta}$ such that

- $\sigma_{\eta}$ intersects $\alpha(\partial Q)$ exactly once,

- this intersection lies on $\eta$ and it is transverse to $F$ and

- both end points of $\sigma_{\eta}$ lie in the contact region.

Because both unstable leaves of $\alpha_{w_{1}}$ on $\gamma_{w_{1} \ldots w_{l}}$ can be extended to Legendrian curves connecting the pseudovertex to $H(\xi)$ such an arc $\sigma_{\eta}$ exists. Now apply (the proof of) Lemma 6.4 to $\sigma_{\eta}$ : After a $C^{\infty}$-small perturbation we obtain a confoliation such that $\sigma_{\eta}$ is in the contact region and the characteristic foliation on $F$ near $\sigma_{\eta} \cap \eta$ remains unchanged. The characteristic foliation has changed near other intersection points of $F$ 
with $\sigma_{\eta}$. By the choice of $\sigma_{\eta}$ these changes affect only the characteristic foliation of $F$ away from $\alpha(Q)$ and therefore preserve the overtwisted star.

After a small modification of $F$ near $\sigma_{\eta} \cap \eta$ we can achieve that the unstable leaf of $\alpha\left(w_{2}\right)$ which corresponded to $\eta$ before the perturbation of $F$ now ends at the negative elliptic singularity which is connected to $\alpha\left(w_{1}\right)$ by a Legendrian path containing an unstable leaf of $\alpha\left(w_{1}\right)$ which does not meet $\gamma_{w_{1} \ldots w_{l}}$ except at the endpoints. This reduces the number of pseudovertices which are identified by $\alpha$ while the overtwisted star is preserved.

It remains to deal with pseudovertices $w_{1}, w_{2}$ such that $\alpha\left(w_{1}\right)=\alpha\left(w_{2}\right)=: y$. The $\omega$-limit sets of the stable leaves of $y$ are negative elliptic singularities $y_{1}, y_{2}$ in $\alpha(\partial Q)$ and we may assume that these singularities are contained in $H(\xi)$ (because they are $\omega$-limit sets, they do not lie in the interior of the foliated part of $\xi$ ).

We eliminate $y_{1}$ and $y$ using Lemma 3.5. This reduces the number of edges of the polygon which are identified unless $y_{1}=y_{2}$. The case when $y_{1}=y_{2}$ requires slightly more work:

After perturbing the surface on a neighbourhood of $y_{1}$ we may assume that the two unstable leaves of $y$ form a smooth closed Legendrian curve $\gamma^{\prime}$. We eliminate $y_{1}, y$ such that $\gamma^{\prime}$ is a closed leaf of the characteristic foliation on the resulting surface. We obtained a Legendrian polygon $\left(Q^{\prime}, V^{\prime}, \alpha^{\prime}\right)$ on a surface $F^{\prime}$ with $Q^{\prime} \simeq D^{2}$ and $V^{\prime}$ consists of all vertices of $Q^{\prime}$ which were mapped to $y_{1}$ by $\alpha^{\prime}$. By construction $\gamma_{v^{\prime}}=\gamma^{\prime}$ for all $v^{\prime} \in V^{\prime}$.

Since $y_{1} \in H\left(\xi^{\prime}\right)$ we can approximate $\xi^{\prime}$ by a confoliation $\xi^{\prime \prime}$ which coincides with $\xi^{\prime}$ outside a tubular neighbourhood of $\gamma^{\prime}$ and is a contact structure near $\gamma^{\prime}$. This can be done without changing the characteristic foliation on the surface by Lemma 6.4.

Next we apply a standard procedure from contact topology called folding to $\gamma^{\prime}$. This is described in [22, page 325]. We obtain a surface $F^{\prime \prime}$ which contains an overtwisted $\operatorname{star}\left(Q^{\prime \prime}, V^{\prime \prime}, \alpha^{\prime \prime}\right)$ such that $V^{\prime}$ consists of two elements with $Q^{\prime \prime} \simeq Q^{\prime}, V^{\prime \prime}=V^{\prime}$ but now elements of $V^{\prime \prime}$ correspond to different $\omega$-limit sets depending on which side of $\gamma^{\prime}$ the corresponding leaves of $\alpha(Q)$ accumulated.

In order to continue we create a pair of negative singularities along the closed leaves in $\overline{\alpha^{\prime \prime}\left(Q^{\prime \prime}\right)}$. We eliminate all pseudovertices successively and we obtain a confoliation $\tilde{\xi}$ on $M$ together with an overtwisted $\operatorname{star}(\widetilde{Q}, \widetilde{V}=\varnothing, \widetilde{\alpha})$ on a surface $\widetilde{F}$ which has no virtual vertices and is injective as a Legendrian polygon. $\widetilde{\alpha}$ becomes injective after finitely many perturbations of $\widetilde{F}$ as in Figure 10.

Because $\widetilde{\alpha}(\partial \widetilde{Q})$ passes through the contact region of $\widetilde{\xi}^{\prime}$ the disc $D=\widetilde{\alpha}(\widetilde{Q})$ violates Definition 1.3. This concludes the proof of the theorem. 
This proof can be modified to yield a proof of Theorem 1.4 using only the well known fact that symplectically fillable contact structures are tight without referring to results of R Hind in [20] which are used in [10]. Let us outline the argument.

Given a disc $D$ as in Definition 1.3 assume first that the holonomy of $\partial D$ in $D$ is nontrivial. We try to follow the construction above to find a confoliation $\xi^{\prime}$ such that $\partial D$ remains Legendrian and $\xi^{\prime}$ is $C^{0}$-close to $\xi$. This attempt must fail since otherwise we could continue to modify $\xi^{\prime}$ into a symplectically fillable contact structure such that $D$ becomes an overtwisted disc. This contradicts the fact that symplectically fillable contact structures are tight.

The point at which the above construction breaks down is the application of Remark 6.6 in the case when $\partial D$ bounds a disc $D^{\prime}$ in the maximal surface which contains $\partial D$ and is tangent to the confoliation. In order to show that $e(\xi)\left[D \cup D^{\prime}\right]=0$ one chooses an embedded sphere $S$ close (and homologous) to $D \cup D^{\prime}$. Then $e(\xi)[S]=0$ follows from the tightness of contact structures that are $C^{0}$-close to the original confoliation.

It remains to treat the case when the holonomy of $\partial D$ in $D$ is trivial. Then one has to show that either $\partial D$ is a vanishing cycle (cf Chapter 9 in [6]) or one can replace $D$ by a smaller disc which has Legendrian boundary along which the holonomy of the characteristic foliation on the disc is not trivial. If $\partial D$ is a vanishing cycle, then one uses results due to Novikov [27] to establish the existence of a solid torus whose boundary $T$ is a leaf of the confoliation. This contradicts $\int_{T} \omega>0$ because this inequality means that $T$ represents a nontrivial homology class.

\section{References}

[1] B Aebischer, M Borer, M Kälin, C Leuenberger, H M Reimann, Symplectic geometry, Progress in Math. 124, Birkhäuser Verlag, Basel (1994) MR1296462 An introduction based on the seminar in Bern, 1992

[2] S J Altschuler, L F Wu, On deforming confoliations, J. Differential Geom. 54 (2000) 75-97 MR1815412

[3] S K Aranson, G R Belitsky, $\mathbf{E} \mathbf{V}$ Zhuzhoma, Introduction to the qualitative theory of dynamical systems on surfaces, Translations of Math. Monogr. 153, Amer. Math. Soc. (1996) MR1400885 Translated from the Russian manuscript by H H McFaden

[4] D Bennequin, Entrelacements et équations de Pfaff, from: "Third Schnepfenried geometry conference, Vol. 1 (Schnepfenried, 1982)”, Astérisque 107, Soc. Math. France (1983) 87-161 MR753131

[5] A Candel, L Conlon, Foliations. I, Graduate Studies in Math. 23, Amer. Math. Soc. (2000) MR1732868 
[6] A Candel, L Conlon, Foliations. II, Graduate Studies in Math. 60, Amer. Math. Soc. (2003) MR1994394

[7] V Colin, Chirurgies d'indice un et isotopies de sphères dans les variétés de contact tendues, C. R. Acad. Sci. Paris Sér. I Math. 324 (1997) 659-663 MR1447038

[8] V Colin, Recollement de variétés de contact tendues, Bull. Soc. Math. France 127 (1999) 43-69 MR1700468

[9] Y Eliashberg, Contact 3-manifolds twenty years since J Martinet's work, Ann. Inst. Fourier (Grenoble) 42 (1992) 165-192 MR1162559

[10] Y Eliashberg, W P Thurston, Confoliations, Univ. Lecture Series 13, Amer. Math. Soc. (1998) MR1483314

[11] J B Etnyre, Approximation of foliations by contact structures, in preparation

[12] J B Etnyre, Introductory lectures on contact geometry, from: "Topology and geometry of manifolds (Athens, GA, 2001)”, (G Matić, C McCrory, editors), Proc. Sympos. Pure Math. 71, Amer. Math. Soc. (2003) 81-107 MR2024631

[13] J B Etnyre, Contact structures on 3-manifolds are deformations of foliations, Math. Res. Lett. 14 (2007) 775-779 MR2350123

[14] J B Etnyre, Contact geometry in low dimensional topology, from: "Low dimensional topology”, (T S Mrowka, P S Ozsváth, editors), IAS/Park City Math. Ser. 15, Amer. Math. Soc. (2009) 229-264 MR2503497

[15] H Geiges, An introduction to contact topology, Cambridge Studies in Advanced Math. 109, Cambridge Univ. Press (2008) MR2397738

[16] E Giroux, Convexité en topologie de contact, Comment. Math. Helv. 66 (1991) 637-677 MR1129802

[17] E Giroux, Structures de contact en dimension trois et bifurcations des feuilletages de surfaces, Invent. Math. 141 (2000) 615-689 MR1779622

[18] C Gutiérrez, Smoothing continuous flows on two-manifolds and recurrences, Ergodic Theory Dynam. Systems 6 (1986) 17-44 MR837974

[19] G Hector, U Hirsch, Introduction to the geometry of foliations. Part B. Foliations of codimension one, second edition, Aspects of Math. E3, Friedr. Vieweg \& Sohn, Braunschweig (1987) MR1110794

[20] R Hind, Filling by holomorphic disks with weakly pseudoconvex boundary conditions, Geom. Funct. Anal. 7 (1997) 462-495 MR1466335

[21] M W Hirsch, Differential topology, Graduate Texts in Math. 33, Springer, New York (1976) MR0448362

[22] K Honda, On the classification of tight contact structures. I, Geom. Topol. 4 (2000) 309-368 MR1786111 
[23] A Katok, B Hasselblatt, Introduction to the modern theory of dynamical systems, Encycl. of Math. and its Appl. 54, Cambridge Univ. Press (1995) MR1326374 With a supplementary chapter by Katok and L Mendoza

[24] J E Marsden, M McCracken, The Hopf bifurcation and its applications, Appl. Math. Sci. 19, Springer, New York (1976) MR0494309

[25] A Mori, A note on Thurston-Winkelnkemper's construction of contact forms on 3manifolds, Osaka J. Math. 39 (2002) 1-11 MR1883910

[26] I Nikolaev, E Zhuzhoma, Flows on 2-dimensional manifolds. An overview, Lecture Notes in Math. 1705, Springer, Berlin (1999) MR1707298

[27] S P Novikov, The topology of foliations, Trudy Moskov. Mat. Obšč. 14 (1965) 248-278 MR0200938

[28] C Petronio, A theorem of Eliashberg and Thurston on foliations and contact structures, Notes of Courses Given by Teachers at the School, Scuola Normale Superiore, Pisa (1997) MR1642484

[29] R Roussarie, Plongements dans les variétés feuilletées et classification de feuilletages sans holonomie, Inst. Hautes Études Sci. Publ. Math. (1974) 101-141 MR0358809

[30] R Sacksteder, Foliations and pseudogroups, Amer. J. Math. 87 (1965) 79-102 MR0174061

[31] W P Thurston, A norm for the homology of 3-manifolds, Mem. Amer. Math. Soc. 59 no. 339, Amer. Math. Soc. (1986) MR823443

Max-Planck-Institut für Mathematik

Vivatsgasse 7, D-53129 Bonn, Germany

tvogel@mpim-bonn.mpg.de

Proposed: Yasha Eliashberg

Seconded: Danny Calegari, Leonid Polterovich

Received: 30 March 2009

Revised: 16 June 2010 